Supporting Information

\title{
Alkali Metal Ions Dictate the Structure and Reactivity of an Iron(II) Imido Complex
}

\author{
Yafei Gao, Maren Pink, and Jeremy M. Smith* \\ Department of Chemistry, Indiana University, 800 East Kirkwood Avenue, Bloomington, Indiana \\ 47405, United States
}




\section{Contents}

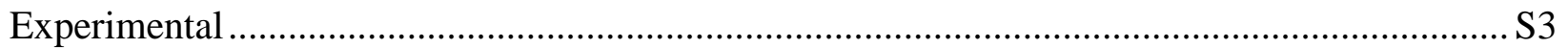

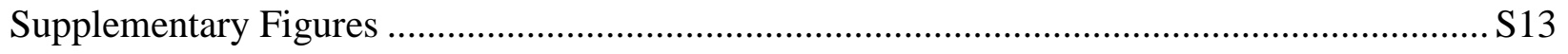

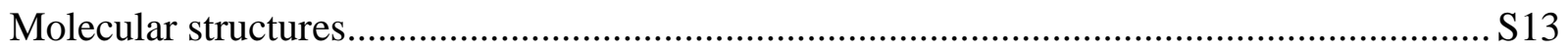

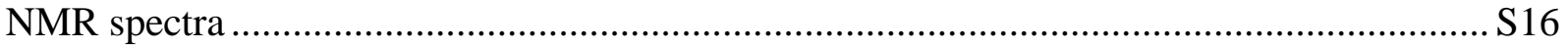

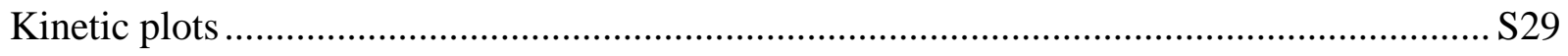

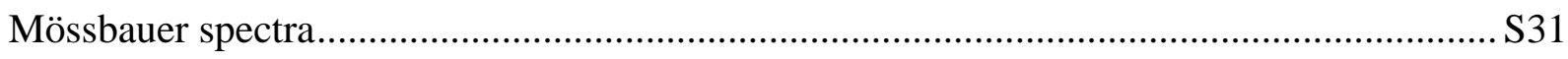

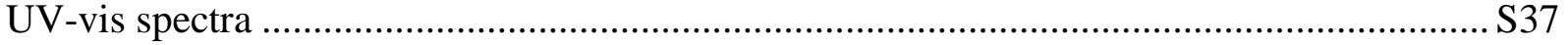

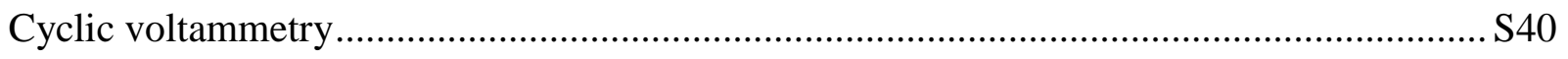

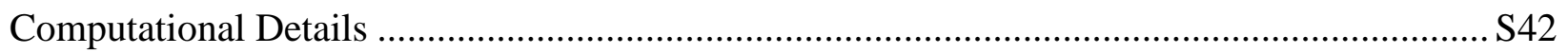

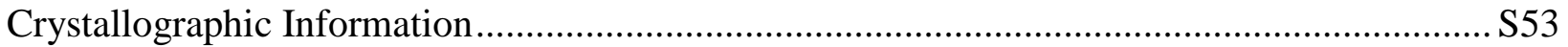

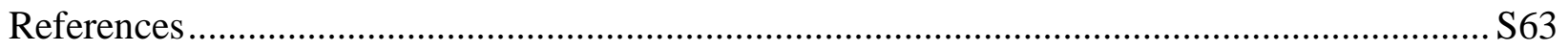




\section{Experimental}

General Considerations. All manipulations were performed under a nitrogen atmosphere by standard Schlenk techniques or in an MBraun glove box. Glassware was dried at $130{ }^{\circ} \mathrm{C}$ overnight before cooling under a dynamic vacuum in an antechamber. Manipulations involving high vacuum and reactive gases were performed using standard Schlenk techniques in a single-manifold glass line. Diethyl ether $\left(\mathrm{Et}_{2} \mathrm{O}\right)$, tetrahydrofuran (THF), toluene, and pentane were purified by a Glass Contour solvent purification system. Celite was dried overnight at $130{ }^{\circ} \mathrm{C}$ under vacuum. Potassium graphite $\left(\mathrm{KC}_{8}\right)^{1},\left[\mathrm{Ph}_{2} \mathrm{~B}\left({ }^{t} \mathrm{BuIm}\right)_{2} \mathrm{FeNDipp}\right](\mathbf{1})^{2}$, 2,6-lutidinium chloride ${ }^{3}$ and 1,4cyclohexadiene- $d_{8}{ }^{4}$ were synthesized according to the literature procedures. All other chemicals were purchased and used as received. ${ }^{1} \mathrm{H}$ NMR spectroscopic data were recorded on Varian spectrometers. Solution magnetic susceptibilities were determined by Evans' method. ${ }^{5}$ UV-Vis spectroscopic data were collected on an Agilent Technologies Cary 60 UV-Vis instrument. Cyclic voltammograms were measured on a CHI 600D potentiostat in THF solution using a sweep rate of $200 \mathrm{mV} / \mathrm{s}$, with a glassy carbon working electrode, $0.1 \mathrm{M}\left[{ }^{n} \mathrm{Bu}_{4} \mathrm{~N}\right]\left[\mathrm{PF}_{6}\right]$ supporting electrolyte, and Ag wire as reference electrode.

${ }^{57} \mathrm{Fe}$ Mössbauer spectra were recorded on a SEE Co spectrometer. The sample temperature was controlled using a SVT-400 Dewar from Janis equipped with a Lake Shore 255 Temperature Controller. The isomer shifts are reported relative to the centroid of the spectrum of $\alpha$-Fe at 298 K. Samples were prepared by grinding crystallized material into a fine powder and then mounting in a cup, plugged with a fitted O-ring sealed cap. Data analysis was performed using the program $\mathrm{WMOSS}^{6}$ and quadrupole doublets were fitted to Lorentzian lineshapes. Elemental analysis was conducted by Midwest Microlab, LLC (Indianapolis, IN). 


\section{Synthesis of Complexes}

[Ph2B $\left({ }^{t} \mathbf{B u I m}\right)_{2}$ FeNDippLi(THF) 2$]$ (2). A vial was charged with $\mathrm{Ph}{ }_{2} \mathrm{~B}\left({ }^{t} \mathrm{BuIm}\right)_{2}$ FeNDipp (1) (550 $\mathrm{mg}, 0.86 \mathrm{mmol})$ and $\mathrm{THF}(5 \mathrm{~mL})$. The solution was cooled to $-78{ }^{\circ} \mathrm{C}$. Solid lithium $(8 \mathrm{mg}, 1.11$ mmol) was added, the mixture was allowed to warm to room temperature and stirred overnight. The solution turned red over the course of the reaction. The solution was filtered through Celite and concentrated under vacuum to afford a red residue that was washed with pentane $(3 \times 5 \mathrm{~mL})$ to give $\left[\mathrm{Ph}_{2} \mathrm{~B}\left({ }^{t} \mathrm{BuIm}\right)_{2} \mathrm{FeNDippLi}(\mathrm{THF})_{2}\right]$ (2) as a red powder (401 $\mathrm{mg}, 72 \%$ yield). Crystals suitable for X-ray diffraction were grown at $-35{ }^{\circ} \mathrm{C}$ from a concentrated THF solution that was layered with pentane. ${ }^{1} \mathrm{H}$ NMR (400 MHz, THF- $\left.d_{8}, 25^{\circ} \mathrm{C}\right): \delta(\mathrm{ppm}) 110.5(2 \mathrm{H}, \mathrm{Im}-H), 46.1(5 \mathrm{H}$, $\left.\mathrm{C}_{6} H_{5}\right), 34.9\left(5 \mathrm{H}, \mathrm{C}_{6} H_{5}\right), 21.5(2 \mathrm{H}, \mathrm{Im}-\mathrm{H}),-5.5\left(3 \mathrm{H}, \mathrm{C}_{6} \mathrm{H}_{3}\right),-22.5\left(12 \mathrm{H}, \mathrm{CH}\left(\mathrm{CH}_{3}\right)_{2}\right),-93.8(18 \mathrm{H}$, $\left.\mathrm{C}\left(\mathrm{CH}_{3}\right)_{3}\right),-96.4\left(1 \mathrm{H}, \mathrm{CH}\left(\mathrm{CH}_{3}\right)_{2}\right),-105.1\left(1 \mathrm{H}, \mathrm{CH}\left(\mathrm{CH}_{3}\right)_{2}\right) . \mu_{\mathrm{eff}}\left(\right.$ Evans', THF- $\left.d_{8}, 25{ }^{\circ} \mathrm{C}\right): 5.0(1) \mu_{\mathrm{B}}$. UV-vis (THF, $25{ }^{\circ} \mathrm{C}$ ): $\lambda_{\max }, \mathrm{nm}\left(\varepsilon, \mathrm{M}^{-1} \mathrm{~cm}^{-1}\right)=269$ (19000), 297 (15000), 315 (13800). Anal. Calcd. for $\mathrm{C}_{46} \mathrm{H}_{65} \mathrm{BFeLiN}_{5} \mathrm{O}_{2}$ : C 69.62, H 8.26, N 8.82. Found C 68.85, H 8.05, N 8.92.

$\left[\mathbf{P h}{ }_{2} \mathrm{~B}\left({ }^{t} \mathbf{B u I m}\right)_{2}\right.$ FeNDippNa(THF)3] (3). A vial was charged with $\mathrm{Ph}_{2} \mathrm{~B}\left({ }^{t} \mathrm{BuIm}\right)_{2} \mathrm{FeNDipp}(\mathbf{1})$ (480 $\mathrm{mg}, 0.75 \mathrm{mmol})$ and THF (5 mL). The solution was cooled to $-78{ }^{\circ} \mathrm{C}$. Solid sodium $(22 \mathrm{mg}, 0.97$ mmol) was added, the mixture was allowed to warm to room temperature and stirred overnight. The solution turned red over the course of the reaction. The solution was filtered through Celite and concentrated under vacuum to afford a red residue that was washed with pentane $(3 \times 5 \mathrm{~mL})$ to give $\left[\mathrm{Ph}_{2} \mathrm{~B}\left({ }^{t} \mathrm{BuIm}\right)_{2} \mathrm{FeNDippNa}(\mathrm{THF})_{3}\right](\mathbf{3})$ as a red powder $(370 \mathrm{mg}, 74 \%$ yield $)$. Crystals suitable for X-ray diffraction were grown at $-35{ }^{\circ} \mathrm{C}$ from a concentrated THF solution that was layered with pentane. ${ }^{1} \mathrm{H}$ NMR $\left(400 \mathrm{MHz}, \mathrm{THF}-d_{8}, 25{ }^{\circ} \mathrm{C}\right): \delta(\mathrm{ppm}) 86.9(2 \mathrm{H}, \mathrm{Im}-H), 66.6(1 \mathrm{H}$, $\left.\mathrm{C}_{6} H_{3}\right), 35.1(2 \mathrm{H}, \mathrm{Im}-\mathrm{H}), 10.9\left(12 \mathrm{H}, \mathrm{CH}\left(\mathrm{CH}_{3}\right)_{2}\right), 1.5\left(1 \mathrm{H}, \mathrm{C}_{6} \mathrm{H}_{3}\right), 1.1\left(1 \mathrm{H}, \mathrm{C}_{6} \mathrm{H}_{3}\right),-0.4\left(4 \mathrm{H}, \mathrm{C}_{6} \mathrm{H}_{5}\right)$, 
$-1.9\left(4 \mathrm{H}, \mathrm{C}_{6} H_{5}\right),-2.6\left(2 \mathrm{H}, \mathrm{C}_{6} H_{5}\right),-78.2\left(18 \mathrm{H}, \mathrm{C}\left(\mathrm{CH}_{3}\right)_{3}\right),-114.2\left(1 \mathrm{H}, \mathrm{CH}\left(\mathrm{CH}_{3}\right)_{2}\right),-119.6(1 \mathrm{H}$, $\left.\mathrm{CH}\left(\mathrm{CH}_{3}\right)_{2}\right) . \mu_{\text {eff }}$ (Evans', THF- $\left.d_{8}, 25^{\circ} \mathrm{C}\right): 5.1(1) \mu_{\mathrm{B}}$. UV-vis (THF, $\left.25^{\circ} \mathrm{C}\right): \lambda_{\max }, \mathrm{nm}\left(\varepsilon, \mathrm{M}^{-1} \mathrm{~cm}^{-1}\right)$ $=268$ (18000), 293 (13700), 317 (12700). Anal. Calcd. for $\mathrm{C}_{42} \mathrm{H}_{57} \mathrm{BFeN}_{5} \mathrm{NaO}: \mathrm{C} 68.39, \mathrm{H} 7.79, \mathrm{~N}$ 9.50. Found C 67.78, H 7.79, N 9.81.

$\left[\mathbf{P h}_{2} \mathbf{B}\left({ }^{t} \mathbf{B u I m}\right)_{2} \mathbf{F e N D i p p K}\right]_{2}$ (4). A vial was charged with $\mathrm{Ph}_{2} \mathrm{~B}\left({ }^{t} \mathrm{BuIm}\right)_{2} \mathrm{FeNDipp}(\mathbf{1})$ (270 mg, $0.42 \mathrm{mmol})$ and THF $(5 \mathrm{~mL})$. The solution was cooled to $-78^{\circ} \mathrm{C}$. Solid $\mathrm{KC}_{8}(68 \mathrm{mg}, 0.50 \mathrm{mmol})$ was added, the mixture was allowed to warm to room temperature and stirred overnight. The solution turned red over the course of the reaction. The solution was filtered through Celite and concentrated under vacuum to afford a red residue that was washed with pentane $(3 \times 5 \mathrm{~mL})$ to give $\left[\mathrm{Ph}_{2} \mathrm{~B}\left({ }^{t} \mathrm{BuIm}\right)_{2} \mathrm{FeNDippK}\right]_{2}(\mathbf{4})$ as a red powder $(231 \mathrm{mg}, 81 \%$ yield $)$. Crystals suitable for $\mathrm{X}$ ray diffraction were grown at $-35{ }^{\circ} \mathrm{C}$ from a concentrated THF solution that was layered with pentane. ${ }^{1} \mathrm{H}$ NMR $\left(400 \mathrm{MHz}, \mathrm{THF}-d_{8}, 25{ }^{\circ} \mathrm{C}\right): \delta(\mathrm{ppm}) 117.0\left(1 \mathrm{H}, \mathrm{CH}\left(\mathrm{CH}_{3}\right)_{2}\right), 95.3(2 \mathrm{H}, \mathrm{Im}-H)$, $80.5(2 \mathrm{H}, \mathrm{Im}-\mathrm{H}), 32.5\left(3 \mathrm{H}, \mathrm{C}_{6} \mathrm{H}_{3}\right), 14.5\left(12 \mathrm{H}, \mathrm{CH}\left(\mathrm{CH}_{3}\right)_{2}\right),-0.9\left(4 \mathrm{H}, \mathrm{C}_{6} \mathrm{H}_{5}\right),-2.7\left(5 \mathrm{H}, \mathrm{C}_{6} \mathrm{H}_{5}\right),-3.4$ $\left(1 \mathrm{H}, \mathrm{C}_{6} H_{5}\right),-76.9\left(18 \mathrm{H}, \mathrm{C}\left(\mathrm{CH}_{3}\right)_{3}\right),-106.4\left(1 \mathrm{H}, \mathrm{CH}\left(\mathrm{CH}_{3}\right)_{2}\right) . \mu_{\mathrm{eff}}\left(\right.$ Evans', THF- $\left.d_{8}, 25{ }^{\circ} \mathrm{C}\right): 6.3(1)$

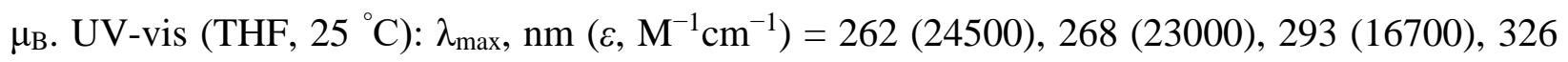
(16500). Anal. Calcd. for $\mathrm{C}_{38} \mathrm{H}_{49} \mathrm{BFeKN}_{5}$ : C 66.96, H 7.25, N 10.28. Found C 65.94, H 7.76, N 9.64 .

$\left[\mathbf{P h}_{2} \mathbf{B}\left({ }^{t} \mathrm{BuIm}\right)_{2}\right.$ FeNDipp][K(18-C-6)THF 2$] \quad$ (5). A vial was charged with $\left[\mathrm{Ph}{ }_{2} \mathrm{~B}\left({ }^{t} \mathrm{BuIm}\right){ }_{2} \mathrm{FeNDippK}\right](4)$ (640 mg, $\left.0.94 \mathrm{mmol}\right)$ and THF (5 mL). Solid 18-crown-6 (248 mg, $0.94 \mathrm{mmol}$ ) was added at room temperature and the mixture was allowed to stir for 4 hours. The THF was removed under vacuum, affording a red residue that was washed with pentane $(3 \times 5 \mathrm{~mL})$ 
to give the previously reported complex $\left[\mathrm{Ph}_{2} \mathrm{~B}\left({ }^{t} \mathrm{BuIm}\right)_{2} \mathrm{FeNDipp}\right]\left[\mathrm{K}(18-\mathrm{C}-6) \mathrm{THF}_{2}\right](\mathbf{5})$ as a red powder (812 mg, 79\% yield). The identity of the sample was confirmed by ${ }^{1} \mathrm{H}$ NMR. ${ }^{2}$

[Ph $2 \mathrm{~B}\left({ }^{t} \mathbf{B u I m}\right)_{2}$ FeNHDipp] (6). A vial was charged with $\left[\mathrm{Ph}{ }_{2} \mathrm{~B}\left({ }^{t} \mathrm{BuIm}\right)_{2} \mathrm{FeNDippK}\right](4)(234 \mathrm{mg}$, $0.34 \mathrm{mmol})$ and THF (5 mL). The solution was cooled to $-78^{\circ} \mathrm{C}$. Solid 2,6 -lutidinium chloride (49 $\mathrm{mg}, 0.34 \mathrm{mmol}$ ) was added to the solution and the mixture was allowed to warm to room temperature. The solution turned brownish yellow over the course overnight stirring. The solution was concentrated under vacuum to afford a brownish yellow residue that was extracted with $\mathrm{Et}_{2} \mathrm{O}$ $(5 \mathrm{~mL})$ and filtered through Celite to give a clear yellow solution. After removal of the solvent under vacuum, complex $\left[\mathrm{Ph}_{2} \mathrm{~B}\left({ }^{t} \mathrm{BuIm}\right)_{2}\right.$ FeNHDipp $](6)$ was obtained as a yellow powder $(175 \mathrm{mg}$, $80 \%$ yield). Crystals suitable for $\mathrm{X}$-ray diffraction were grown at $-35^{\circ} \mathrm{C}$ from a concentrated $\mathrm{Et}_{2} \mathrm{O}$ solution. ${ }^{1} \mathrm{H}$ NMR $\left(400 \mathrm{MHz}, \mathrm{C}_{6} \mathrm{D}_{6}, 25^{\circ} \mathrm{C}\right): \delta(\mathrm{ppm}) 106.0(1 \mathrm{H}, \mathrm{Im}-H), 86.9(1 \mathrm{H}, \mathrm{Im}-H), 39.8(1 \mathrm{H}$, $\left.\mathrm{C}_{6} \mathrm{H}_{3}\right), 24.6\left(2 \mathrm{H}, \mathrm{C}_{6} \mathrm{H}_{3}\right), 11.9\left(12 \mathrm{H}, \mathrm{CH}\left(\mathrm{CH}_{3}\right)_{2}\right), 3.7\left(4 \mathrm{H}, \mathrm{C}_{6} \mathrm{H}_{5}\right), 3.0\left(4 \mathrm{H}, \mathrm{C}_{6} \mathrm{H}_{5}\right), 1.2\left(2 \mathrm{H}, \mathrm{C}_{6} H_{5}\right)$, $0.2(2 \mathrm{H}, \mathrm{Im}-\mathrm{H}),-11.6\left(1 \mathrm{H}, \mathrm{CH}\left(\mathrm{CH}_{3}\right)_{2}\right),-48.7\left(1 \mathrm{H}, \mathrm{CH}\left(\mathrm{CH}_{3}\right)_{2}\right),-57.7\left(18 \mathrm{H}, \mathrm{C}\left(\mathrm{CH}_{3}\right)_{3}\right) . \mu \mu_{\mathrm{eff}}($ Evans', $\left.\mathrm{C}_{6} \mathrm{D}_{6}, 25{ }^{\circ} \mathrm{C}\right): 5.4(1) \mu_{\mathrm{B}} . \mathrm{UV}$-vis $\left(\mathrm{THF}, 25{ }^{\circ} \mathrm{C}\right): \lambda_{\max }, \mathrm{nm}\left(\varepsilon, \mathrm{M}^{-1} \mathrm{~cm}^{-1}\right)=289(13300), 319(8800)$, 427 (1900). Anal. Calcd. for $\mathrm{C}_{38} \mathrm{H}_{50} \mathrm{BFeN}_{5}$ : C 70.93, H 7.83, N 10.88. Found C 71.24, H 7.66, N 11.00 .

Complex 6 can similarly be prepared from 2 and 3 in $82 \%$ and 79\% isolated yields, respectively.

$\left[\mathbf{P h}_{2} \mathbf{B}\left({ }^{t} \mathbf{B u I m}\right)_{2} \mathbf{F e N}\left(\mathbf{C H}_{2} \mathbf{P h}\right)\right.$ Dipp $]$ (7). A vial was charged with $\left[\mathrm{Ph}_{2} \mathrm{~B}\left({ }^{t} \mathrm{BuIm}\right)_{2} \mathrm{FeNDippK}\right]$ (4) (346 mg, $0.51 \mathrm{mmol}$ ) and THF (5 mL). The solution was cooled to $-78{ }^{\circ} \mathrm{C}$. Liquid Benzyl bromide (87 $\mathrm{mg}, 0.51 \mathrm{mmol}$ ) was added to the solution and the mixture was allowed to warm to room temperature. The solution turned brownish yellow over the course overnight stirring. The solution was concentrated under vacuum to afford a brownish yellow residue that was extracted with $\mathrm{Et}_{2} \mathrm{O}$ 
$(5 \mathrm{~mL})$ and filtered through Celite to give a clear yellow solution. After removal of the solvent under vacuum, complex $\left[\mathrm{Ph}_{2} \mathrm{~B}\left({ }^{t} \mathrm{BuIm}\right)_{2} \mathrm{FeN}\left(\mathrm{CH}_{2} \mathrm{Ph}\right) \mathrm{Dipp}\right]$ (7) was obtained as a yellow powder (320 mg, 86\% yield). Crystals suitable for X-ray diffraction were grown at $-35{ }^{\circ} \mathrm{C}$ from a concentrated $\mathrm{Et}_{2} \mathrm{O}$ solution. ${ }^{1} \mathrm{H}$ NMR (400 MHz, THF- $\left.d_{8}, 25^{\circ} \mathrm{C}\right): \delta(\mathrm{ppm}) 81.7(1 \mathrm{H}), 75.5(1 \mathrm{H})$, $71.0(1 \mathrm{H}), 58.5(1 \mathrm{H}), 47.8(1 \mathrm{H}), 46.6(1 \mathrm{H}), 32.5(1 \mathrm{H}), 31.8(1 \mathrm{H}), 29.9(2 \mathrm{H}), 26.9(1 \mathrm{H}), 23.8(1 \mathrm{H})$ $22.1(1 \mathrm{H}), 15.6(1 \mathrm{H}), 12.8(1 \mathrm{H}), 7.3(1 \mathrm{H}), 7.2(1 \mathrm{H}), 6.9(1 \mathrm{H}), 6.6(1 \mathrm{H}), 6.1(1 \mathrm{H}), 3.9(1 \mathrm{H}),-4.2$ $(1 \mathrm{H}),-14.8(4 \mathrm{H}),-19.9(1 \mathrm{H}),-31.9(1 \mathrm{H}),-41.9(2 \mathrm{H}),-62.7(1 \mathrm{H}),-71.0\left(9 \mathrm{H}, \mathrm{C}\left(\mathrm{CH}_{3}\right)_{3}\right),-72.7(9 \mathrm{H}$, $\left.\mathrm{C}\left(\mathrm{CH}_{3}\right)_{3}\right),-75.2(3 \mathrm{H}),-108.3(2 \mathrm{H}) . \mu_{\text {eff }}$ (Evans', $\left.\mathrm{C}_{6} \mathrm{D}_{6}, 25^{\circ} \mathrm{C}\right): 5.2(1) \mu_{\mathrm{B}}$. UV-vis (THF, $\left.25^{\circ} \mathrm{C}\right)$ : $\lambda_{\max }, \mathrm{nm}\left(\varepsilon, \mathrm{M}^{-1} \mathrm{~cm}^{-1}\right)=262$ (16700), 269 (14400), 330 (5200). Anal. Calcd. for $\mathrm{C}_{45} \mathrm{H}_{56} \mathrm{BFeN}_{5}: \mathrm{C}$ 73.67, H 7.69, N 9.55. Found C 73.94, H 8.05, N 9.19.

Complex $\mathbf{7}$ can similarly be prepared from $\mathbf{2}$ and $\mathbf{3}$ in 77\% and 81\% isolated yields, respectively.

General procedure for the synthesis of $\left[\mathrm{Ph} 2 \mathrm{~B}\left({ }^{t} \mathrm{BuIm}\right)_{2} \mathrm{Fe}(\mathrm{NHDipp})_{2} \mathrm{M}\right](\mathrm{M}=\mathrm{Li}, \mathrm{Na}, \mathrm{K})(8-\mathrm{M})$. A vial was charged with $\mathrm{Fe}(\mathrm{II})$ imido complexes $\left[\mathrm{Ph}_{2} \mathrm{~B}\left({ }^{t} \mathrm{BuIm}\right)_{2} \mathrm{FeNDippM}\right](\mathrm{M}=\mathrm{Li}, \mathrm{Na}, \mathrm{K})(\mathbf{2}$ 4) $(0.23 \mathrm{mmol})$ and THF $(5 \mathrm{~mL})$. Liquid 2,6-diisopropylaniline $\left(\right.$ DippNH $\left._{2}\right)(40 \mathrm{mg}, 0.23 \mathrm{mmol})$ were added at room temperature and the mixture was allowed to stir for 1 hour. The solution became brownish yellow over the course of the reaction. The THF was removed under vacuum, affording a brownish yellow residue that was washed with pentane $(3 \times 5 \mathrm{~mL})$ to give $\left[\mathrm{Ph}_{2} \mathrm{~B}\left({ }^{t} \mathrm{BuIm}\right)_{2} \mathrm{Fe}(\mathrm{NHDipp})_{2} \mathrm{M}\right](\mathbf{8}-\mathbf{M})$ as a yellow powder. Crystalline material can be obtained at $-35^{\circ} \mathrm{C}$ from a concentrated THF solution that was layered with pentane. The identity of the sample was confirmed by ${ }^{1} \mathrm{H}$ NMR which show identical paramagnetic signals with $\left[\mathrm{Ph}_{2} \mathrm{~B}\left({ }^{t} \mathrm{BuIm}\right)_{2} \mathrm{Fe}(\mathrm{NHDipp})_{2}\right]\left[\mathrm{K}(18-\mathrm{C}-6) \mathrm{THF}_{2}\right](\mathbf{8}-[\mathbf{K}(\mathbf{1 8 - C}-\mathbf{6})])^{2}$ 


\section{Equilibrium constant determination}

In a typical experiment, a J-Young tube was charged with $\left[\mathrm{Ph}_{2} \mathrm{~B}\left({ }^{t} \mathrm{BuIm}\right)_{2} \mathrm{Fe}(\mathrm{NHDipp})_{2} \mathrm{M}\right](\mathbf{8}-\mathbf{M})$ $(\mathrm{M}=\mathrm{Li}, \mathrm{Na}, \mathrm{K})(0.023 \mathrm{mmol})$ and THF- $d_{8}(0.5 \mathrm{~mL})$. Mesitylene $(5 \mathrm{mg}, 0.042 \mathrm{mmol})$ was added as an internal standard. Equilibration was monitored by ${ }^{1} \mathrm{H} \mathrm{NMR}$ (at $25^{\circ} \mathrm{C}$ ), providing equilibrium concentrations of $\left[\mathrm{Ph}_{2} \mathrm{~B}\left({ }^{t} \mathrm{BuIm}\right)_{2} \mathrm{Fe}(\mathrm{NHDipp})_{2} \mathrm{M}\right](\mathbf{8}-\mathbf{M}),\left[\mathrm{Ph}{ }_{2} \mathrm{~B}\left({ }^{t} \mathrm{BuIm}\right)_{2} \mathrm{FeNDippM}\right](\mathbf{2 - 4})$ and $\left[\mathrm{DippNH}_{2}\right]$ according to the integrals of $\mathrm{DippNH}_{2}$ and mesitylene. These concentrations provide the equilibrium constant for these reactions ( $K_{\text {eq }}$ were the averaged data of two runs):

$\begin{array}{ll}{\left[\mathrm{Ph}_{2} \mathrm{~B}\left({ }^{t} \mathrm{BuIm}\right)_{2} \mathrm{Fe}(\mathrm{NHDipp})_{2} \mathrm{~K}\right] \leftrightarrows\left[\mathrm{Ph}{ }_{2} \mathrm{~B}\left({ }^{t} \mathrm{BuIm}\right)_{2} \mathrm{FeNDippK}\right]+\mathrm{H}_{2} \mathrm{NDipp}} & K_{\text {eq }} \approx 6.2(3) \times 10^{-3} \mathrm{~mol} / \mathrm{L} \\ {\left[\mathrm{Ph}{ }_{2} \mathrm{~B}\left({ }^{t} \mathrm{BuIm}\right)_{2} \mathrm{Fe}(\mathrm{NHDipp})_{2} \mathrm{Na}\right] \leftrightarrows\left[\mathrm{Ph}{ }_{2} \mathrm{~B}\left({ }^{t} \mathrm{BuIm}\right)_{2} \mathrm{FeNDippNa}\right]+\mathrm{H}_{2} \mathrm{NDipp}} & K_{\text {eq }} \approx 2.3(6) \times 10^{-3} \mathrm{~mol} / \mathrm{L} \\ {\left[\mathrm{Ph}_{2} \mathrm{~B}\left({ }^{t} \mathrm{BuIm}\right)_{2} \mathrm{Fe}(\mathrm{NHDipp})_{2} \mathrm{Li}\right] \leftrightarrows\left[\mathrm{Ph}_{2} \mathrm{~B}\left({ }^{t} \mathrm{BuIm}\right)_{2} \mathrm{FeNDippLi}\right]+\mathrm{H}_{2} \mathrm{NDipp}} & K_{\text {eq }} \approx 1.9(1) \times 10^{-3} \mathrm{~mol} / \mathrm{L}\end{array}$ 
$\left[\mathrm{Ph}_{2} \mathrm{~B}\left({ }^{t} \mathrm{BuIm}\right)_{2} \mathrm{FeNHDipp}\right]\left[\mathrm{K}(\mathbf{1 8}-\mathrm{C}-6) \mathrm{THF}_{2}\right] \quad(9-[\mathrm{K}(18-\mathrm{C}-6)])$. A vial was charged with $\left[\mathrm{Ph}_{2} \mathrm{~B}\left({ }^{t} \mathrm{BuIm}\right){ }_{2} \mathrm{FeNDipp}\right]\left[\mathrm{K}(18-\mathrm{C}-6) \mathrm{THF}_{2}\right](5)(250 \mathrm{mg}, 0.23 \mathrm{mmol})$ and THF (5 mL). Liquid 1,4cyclohexadiene (10 $\mathrm{mg}, 0.12 \mathrm{mmol})$ was added at room temperature and the mixture was allowed to stir for 1 hour. The volatiles were removed under vacuum, affording a brownish yellow residue that was washed with pentane $(3 \times 5 \mathrm{~mL})$ to give the proposed product $\left[\mathrm{Ph}_{2} \mathrm{~B}\left({ }^{t} \mathrm{BuIm}\right){ }_{2} \mathrm{FeNHDipp}\right]\left[\mathrm{K}(18-\mathrm{C}-6) \mathrm{THF}_{2}\right](\mathbf{9 -}-[\mathbf{K}(\mathbf{1 8 - C}-\mathbf{6})])$ as a brownish yellow powder $(200$ mg, 80\% yield). ${ }^{1} \mathrm{H}$ NMR (400 MHz, THF- $d_{8}, 25^{\circ} \mathrm{C}$ ): $\delta(\mathrm{ppm}) 39.5(1 \mathrm{H}), 21.9(1 \mathrm{H}), 14.7(2 \mathrm{H})$, $8.8\left(12 \mathrm{H}, \mathrm{CH}\left(\mathrm{CH}_{3}\right)_{2}\right), 4.3\left(36 \mathrm{H}, \mathrm{OCH}_{2} \mathrm{CH}_{2} \mathrm{O}\right),-2.7\left(18 \mathrm{H}, \mathrm{C}\left(\mathrm{CH}_{3}\right)_{3}\right),-58.7(1 \mathrm{H})$. The instability of this complex at room temperature does not allow full spectroscopic characterization as it undergoes disproportionation over a period of 12 hours in solution to afford the previously reported complex $\left[\mathrm{Ph}_{2} \mathrm{~B}\left({ }^{t} \mathrm{BuIm}\right)_{2} \mathrm{Fe}(\mathrm{NHDipp})_{2}\right]\left[\mathrm{K}(18-\mathrm{C}-6) \mathrm{THF}_{2}\right](\mathbf{8}-[\mathbf{K}(\mathbf{1 8}-\mathbf{C}-\mathbf{6})]){ }^{2}$

General procedure for determination of KIE of the HAT reactions. In a typical experiment, a J. Young NMR tube was charged with $\mathbf{3 , 4}$ or $\mathbf{5}(0.05 \mathrm{mmol})$, toluene or 1,4-cyclohexadiene (toluene- $d_{8}$ or 1,4-cyclohexadiene- $\left.d_{8}\right)(0.15 \mathrm{mmol})$, THF- $d_{8}(0.5 \mathrm{~mL})$ and a sealed capillary filled with a known amount of ferrocene $(8 \mathrm{mg})$ in THF- $d_{8}$. The solution was monitored by ${ }^{1} \mathrm{H}$ NMR spectroscopy (400 MHz) at $25^{\circ} \mathrm{C}$. The change in concentration of the complexes $\mathbf{3}-\mathbf{5}$ versus reaction time was analyzed to give the KIE, as shown in Figures S32-S34.

Reaction of $\left[\mathrm{Ph}_{2} \mathrm{~B}\left({ }^{t} \mathrm{BuIm}\right)_{2} \mathrm{FeNDipp}\right]\left[\mathrm{K}(18-\mathrm{C}-6) \mathrm{THF}_{2}\right]$ (5) with benzophenone. A vial was charged with $\left[\mathrm{Ph}{ }_{2} \mathrm{~B}\left({ }^{t} \mathrm{BuIm}\right)_{2} \mathrm{FeNDipp}\right]\left[\mathrm{K}(18-\mathrm{C}-6) \mathrm{THF}_{2}\right](\mathbf{5})(132 \mathrm{mg}, 0.12 \mathrm{mmol})$ and THF (5 $\mathrm{mL}$ ). Solid benzophenone ( $22 \mathrm{mg}, 0.12 \mathrm{mmol}$ ) was added at room temperature and the mixture was allowed to stir for 10 minutes. The red solution becomes purple over the course of the reaction. The THF was removed under vacuum, affording the residue that was washed with pentane $(3 \times 5$ 
$\mathrm{mL})$ to give purple $\left[\mathrm{Ph}{ }_{2} \mathrm{~B}\left({ }^{t} \mathrm{BuIm}\right)_{2} \mathrm{Fe}\left(\mathrm{N}(\mathrm{Dipp}) \mathrm{C}(\mathrm{Ph})_{2} \mathrm{O}\right)\right][\mathrm{K}(18-\mathrm{C}-6)](\mathbf{1 0})$, which was immediately analyzed by ${ }^{57} \mathrm{Fe}$ Mössbauer spectroscopy $\left(\delta=0.46 \mathrm{~mm} / \mathrm{s}\right.$ and $\left.\left|\Delta E_{\mathrm{Q}}\right|=2.13 \mathrm{~mm} / \mathrm{s}\right)$ (Figure S42). The instability of this complex at room temperature does not allow full characterization. Please see Figure $\mathrm{S} 26$ for details of its decomposition. Only trace $\mathrm{Ph}_{2} \mathrm{C}=\mathrm{NDipp}$ was observed by ${ }^{1} \mathrm{H}$ NMR spectroscopy. The imine could not be isolated in the large-scale reaction.

General procedure for the reaction of $\left.\left[\mathrm{Ph} 2 \mathrm{~B}\left({ }^{t} \mathrm{BuIm}\right)_{2} \mathrm{FeNDippM}(\mathrm{THF})\right)_{\mathbf{x}}\right](\mathrm{M}=\mathrm{Li}, \mathrm{Na}, \mathrm{K})(2-$ 4) with benzophenone. $A$ vial was charged with $\left[\mathrm{Ph}_{2} \mathrm{~B}\left({ }^{t} \mathrm{BuIm}\right)_{2} \mathrm{FeNDippM}(\mathrm{THF})_{\mathrm{x}}\right](\mathbf{2 - 4})(0.40$ $\mathrm{mmol})$ and THF ( $5 \mathrm{~mL})$. Solid benzophenone $(72 \mathrm{mg}, 0.40 \mathrm{mmol})$ was added at room temperature and the solution was allowed to stir for 10 minutes. Over the course of the reaction, the initially red solution became blue, green and brown for $\mathrm{M}=\mathrm{Li}, \mathrm{Na}$ and $\mathrm{K}$, respectively. The THF was removed under vacuum, and the residue washed with pentane $(3 \times 5 \mathrm{~mL})$ to give solid $\left[\mathrm{Ph}{ }_{2} \mathrm{~B}\left({ }^{t} \mathrm{BuIm}\right)_{2} \mathrm{FeN}(\mathrm{Dipp}) \mathrm{C}(\mathrm{Ph})_{2} \mathrm{OM}(\mathrm{THF})_{\mathrm{x}}\right](\mathbf{1 1}, \mathrm{M}=\mathrm{K} ; \mathbf{1 2}, \mathrm{M}=\mathrm{Na} ; \mathbf{1 3}, \mathrm{M}=\mathrm{Li})$ which were immediately analyzed by ${ }^{57} \mathrm{Fe}$ Mössbauer spectroscopy. The ${ }^{57} \mathrm{Fe}$ Mössbauer spectral parameters of these complexes (Figures S43-S45) are similar to that of $\mathbf{1 0}$ and consistent with those for threecoordinate $S=2 \mathrm{Fe}$ (II) complexes with bis(carbene)borate ligand (Figures S35-S40). The thermal instability of these complexes has limited attempts to full characterization. The decomposition of these complexes to provide of $\mathrm{Ph}_{2} \mathrm{C}=\mathrm{NDipp}$ is shown in Figures S27-S29.

Isolation of $\mathbf{P h}{ }_{2} \mathbf{C}=\mathbf{N D i p p}$. A vial was charged with $\left[\mathrm{Ph}_{2} \mathrm{~B}\left({ }^{t} \mathrm{BuIm}\right)_{2} \mathrm{FeNDippK}\right](\mathbf{4})(415 \mathrm{mg}, 0.61$ $\mathrm{mmol})$ and THF (5 mL). Solid benzophenone $(112 \mathrm{mg}, 0.61 \mathrm{mmol})$ was added at room temperature and the mixture was allowed to stir for overnight. The red solution became brownish yellow over the course of the reaction. The THF was removed under vacuum, affording the residue that was extracted with pentane $(3 \times 5 \mathrm{~mL})$ and filtered through a filter funnel to give a green powder and a 
yellow pentane solution. The pentane was evaporated under vacuum, affording $\mathrm{Ph}_{2} \mathrm{C}=\mathrm{NDipp}$ as a yellow powder $(130 \mathrm{mg}, 62 \%$ yield $) .{ }^{1} \mathrm{H}$ NMR $\left(400 \mathrm{MHz}, \mathrm{THF}-d_{8}, 25^{\circ} \mathrm{C}\right): \delta(\mathrm{ppm}) 7.78(\mathrm{~d}, J=$ 8.0 Hz, 2H), 7.39-7.48 (m, 3H), 7.20-7.22 (m, 3H), $7.10(\mathrm{~d}, J=8.0 \mathrm{~Hz}, 2 \mathrm{H}), 6.94(\mathrm{~d}, J=8.0 \mathrm{~Hz}$, 2H), 6.84-6.88 (m, 1H), $2.90(\mathrm{~m}, 2 \mathrm{H}), 1.10(\mathrm{~d}, J=8.0 \mathrm{~Hz}, 6 \mathrm{H}), 0.96(\mathrm{~d}, J=8.0 \mathrm{~Hz}, 6 \mathrm{H})$. These spectral properties are identical to those reported in the literature. ${ }^{7}$

The isolated green powder was immediately analyzed by ${ }^{57} \mathrm{Fe}$ Mössbauer spectroscopy. The resulting spectrum can be simulated as two major species having spectral parameters $\delta=0.47$ $\mathrm{mm} / \mathrm{s},\left|\Delta E_{\mathrm{Q}}\right|=1.43 \mathrm{~mm} / \mathrm{s}(70 \%)$ and $\delta=0.72 \mathrm{~mm} / \mathrm{s},\left|\Delta E_{\mathrm{Q}}\right|=3.17 \mathrm{~mm} / \mathrm{s}(30 \%)$ (Figure S46) which are consistent with the trigonal planar $S=2 \mathrm{Fe}(\mathrm{II})$ and tetrahedral $S=2 \mathrm{Fe}$ (II) bis(carbene)borate complexes, respectively. These complexes are not stable in solution as assayed by ${ }^{1} \mathrm{H}$ NMR spectroscopy, with the formation of multiple products occurring over several days at room temperature. Multiple attempts to crystallize these complexes as well as the analogous lithium and sodium species were unsuccessful.

These species react with excess $\mathrm{Me}_{3} \mathrm{SiCl}$ to quantitatively afford the iron chloride complex $\left[\mathrm{Ph}_{2} \mathrm{~B}\left({ }^{t} \mathrm{BuIm}\right)_{2} \mathrm{FeCl}(\mathrm{THF})\right],{ }^{8}$ as characterized by ${ }^{1} \mathrm{H}$ NMR spectroscopy. The reaction also affords $\mathrm{Me}_{3} \mathrm{SiOSiMe}_{3}$, which was characterized by ${ }^{29} \mathrm{Si}$ NMR (Figure S31). Together, these observations suggest the formation of iron oxo complex(es), however we cannot definitively rule out other possibilities.

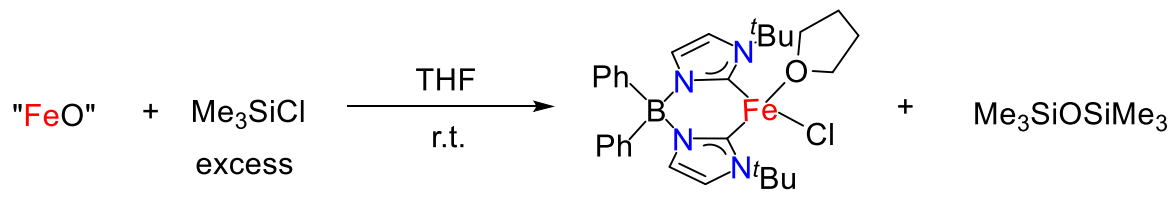


Kinetics of the decomposition of $\left[\mathrm{Ph}_{2} \mathrm{~B}\left({ }^{t} \mathrm{BuIm}\right)_{2} \mathrm{FeN}(\mathrm{Dipp}) \mathrm{C}(\mathrm{Ph})_{2} \mathrm{OM}(\mathrm{THF})_{\mathrm{x}}\right](\mathrm{M}=\mathrm{Li}, \mathrm{Na}, \mathrm{K})$

(11-13). In a typical experiment, a J. Young NMR tube was charged with $\mathbf{2 , 3}$ or $4(0.05 \mathrm{mmol})$, benzophenone $(0.05 \mathrm{mmol})$, THF- $d_{8}(0.5 \mathrm{~mL})$ and ferrocene $(6 \mathrm{mg}, 0.032 \mathrm{mmol})$ as an internal standard. The solution was monitored by ${ }^{1} \mathrm{H}$ NMR spectroscopy (400 MHz) over a period of $10 \mathrm{~h}$, $2 \mathrm{~h}$ and $2 \mathrm{~h}$ for $\mathbf{2}, \mathbf{3}$ and $\mathbf{4}$, respectively. The organic product $\mathrm{Ph}_{2} \mathrm{C}=\mathrm{NDipp}$ was quantified by integration of the ${ }^{1} \mathrm{H}$ NMR spectrum. 


\section{Supplementary Figures}

\section{Molecular structures}

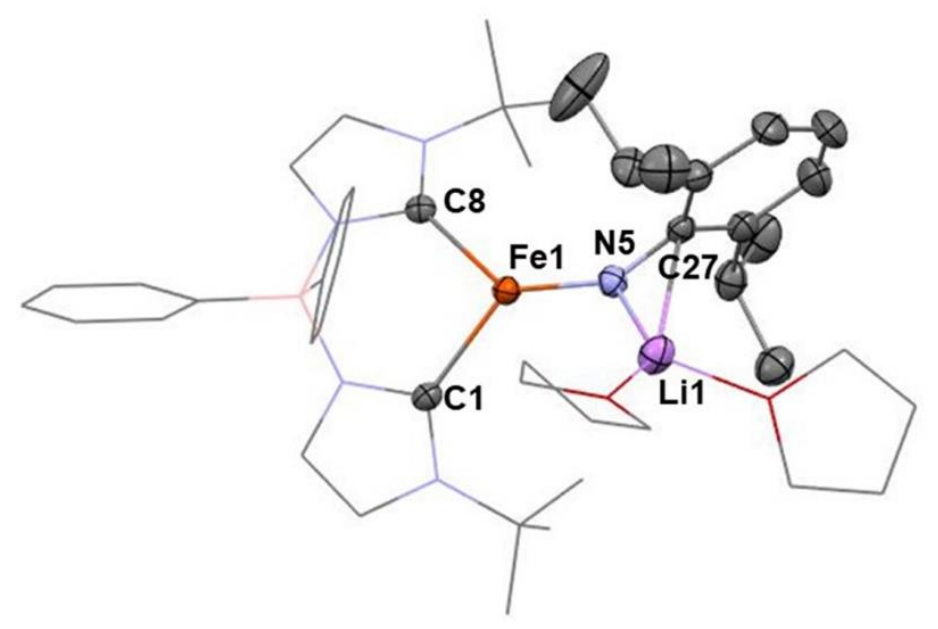

Figure S1. Molecular structure of $\left[\mathrm{Ph}_{2} \mathrm{~B}\left({ }^{t} \mathrm{BuIm}\right)_{2} \mathrm{FeNDippLi}(\mathrm{THF})_{2}\right](\mathbf{2})$, as determined by single crystal X-ray diffraction. Ellipsoids are shown at 50\% probability level, bis(carbene)borate ligand (except for carbene carbon atoms) represented as wireframe. H atoms have been omitted for clarity. Color scheme: C, black; N, blue; B, pink; Fe, orange; Li, purple; O, red. Selected bond distances (A) and angles (deg): Fe1-N5 1.8167(17), Fe1-C1 2.109(2), Fe1-C8 2.110(2), Li1-N5 1.950(4); C1-Fe1-C8 93.04(8), Fe1-N5-C27 143.59(14), C1-Fe1-N5 131.05(8), C8-Fe1-N5 135.72(8).

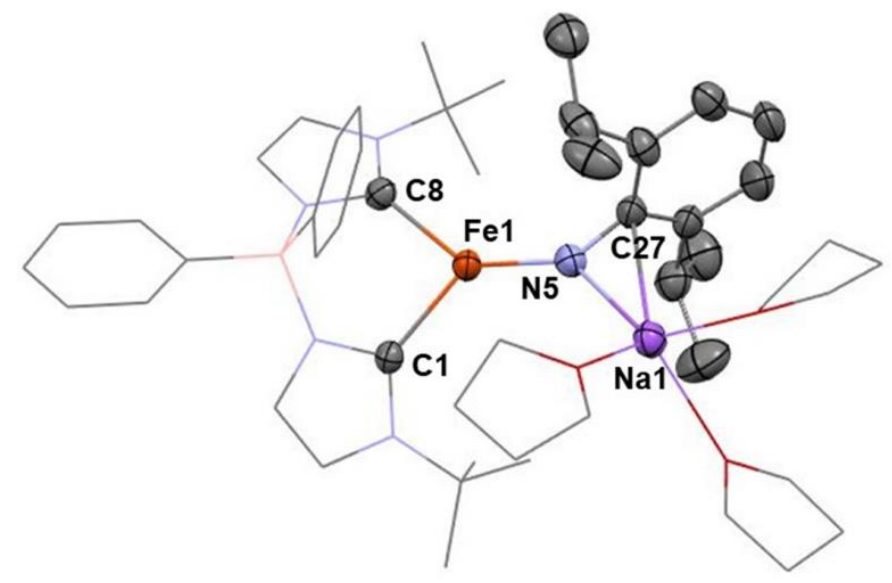

Figure S2. Molecular structure of $\left[\mathrm{Ph}_{2} \mathrm{~B}\left({ }^{t} \mathrm{BuIm}\right)_{2} \mathrm{FeNDippNa}(\mathrm{THF})_{3}\right](\mathbf{3})$, as determined by single crystal X-ray diffraction. Ellipsoids are shown at 50\% probability level, bis(carbene)borate ligand (except for carbene carbon atoms) represented as wireframe. $\mathrm{H}$ atoms have been omitted for clarity. Color scheme: C, black; N, blue; B, pink; Fe, orange; Na, purple; O, red. Selected bond distances (A) and angles (deg): Fe1-N5 1.812(2), Fe1-C1 2.106(3), Fe1-C8 2.087(3), Na1-N5 2.440(3); C1Fe1-C8 93.22(11), Fe1-N5-C27 141.5(2), C1-Fe1-N5 129.40(11), C8-Fe1-N5 137.36(11). 

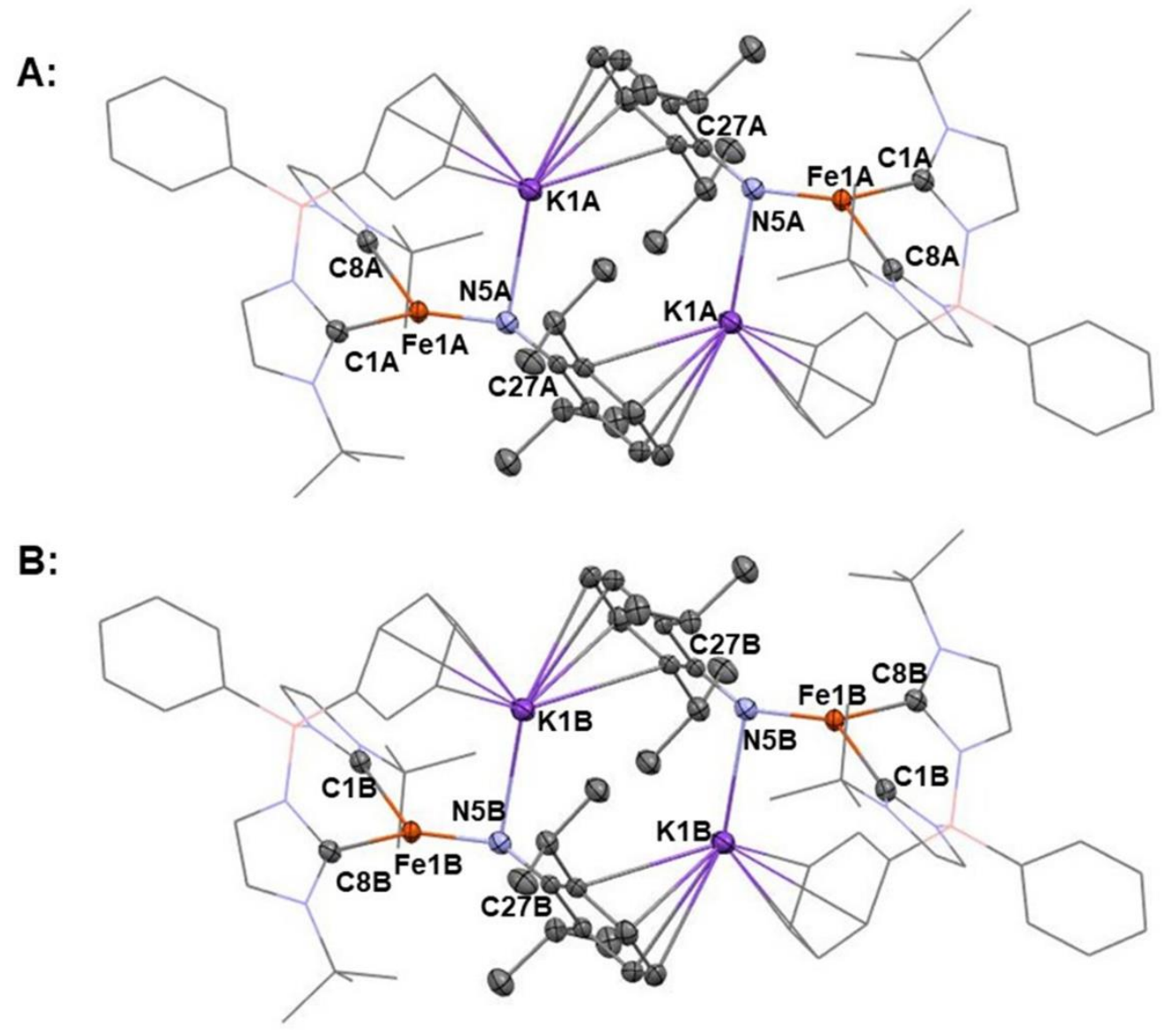

Figure S3. Molecular structure of $\left[\mathrm{Ph}_{2} \mathrm{~B}\left({ }^{t} \mathrm{BuIm}\right)_{2} \mathrm{FeNDippK}\right]_{2}(4)$, as determined by single crystal X-ray diffraction. Ellipsoids are shown at $50 \%$ probability level, bis(carbene)borate ligands (except for carbene carbon atoms) represented as wireframe. Solvent molecules and $\mathrm{H}$ atoms have been omitted for clarity. The compound crystalizes with two sets of molecules in the asymmetric unit. Color scheme: C, black; N, blue; B, pink; Fe, orange; K, purple. Selected bond distances ( $\mathrm{A})$ and angles (deg): Fe1A-N5A 1.792(3), Fe1A-C1A 2.084(4), Fe1A-C8A 2.095(4), K1A-N5A 2.850(3); C1A-Fe1A-C8A 94.69(14), Fe1A-N5A-C27A 154.2(3), C1A-Fe1A-N5A 129.03(15), C8A-Fe1A-N5A 129.36(14) in A. Fe1B-N5B 1.793(3), Fe1B-C1B 2.101(4), Fe1B-C8B 2.098(4), K1B-N5B 2.803(3); C1B-Fe1B-C8B 94.93(14), Fe1B-N5B-C27B 145.9(3), C1B-Fe1B-N5B 126.14(14), C8B-Fe1B-N5B 136.10(14) in B. 


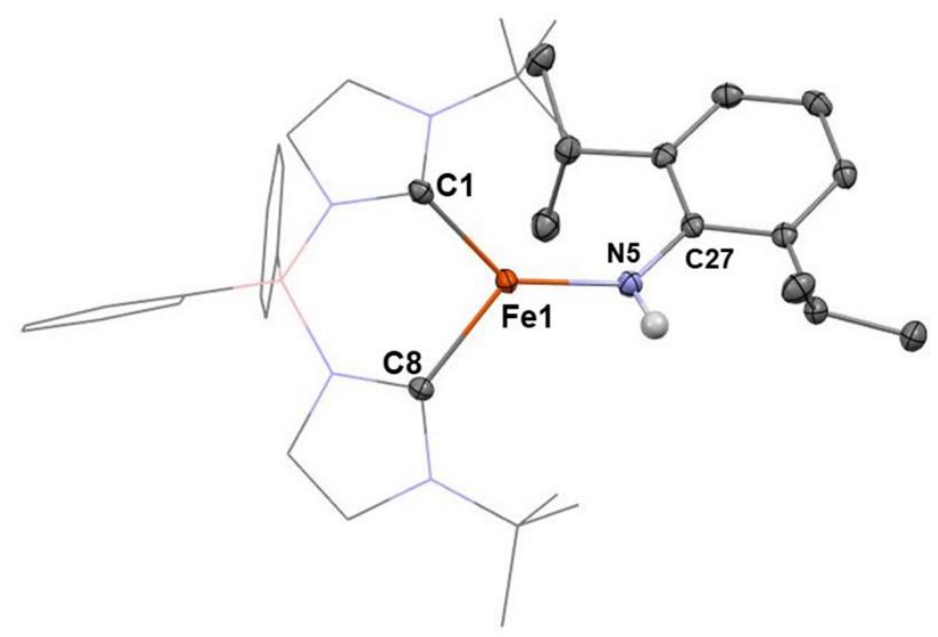

Figure S4. Molecular structure of $\left[\mathrm{Ph}_{2} \mathrm{~B}\left({ }^{t} \mathrm{BuIm}\right)_{2} \mathrm{FeNHDipp}\right](6)$, as determined by single crystal X-ray diffraction. Ellipsoids are shown at 50\% probability level, bis(carbene)borate ligand (except for carbene carbon atoms) represented as wireframes. Solvent molecules and most $\mathrm{H}$ atoms have been omitted for clarity. Color scheme: C, black; N, blue; B, pink; Fe, orange; H, gray. Selected bond distances $(\AA)$ and angles (deg): Fe1-N5 1.9168(14), Fe1-C1 2.0644(16), Fe1-C8 2.0658(16); C1-Fe1-C8 97.41(6), Fe1-N5-C27 137.35(12), C1-Fe1-N5 125.22(6), C8-Fe1-N5 136.86(6).

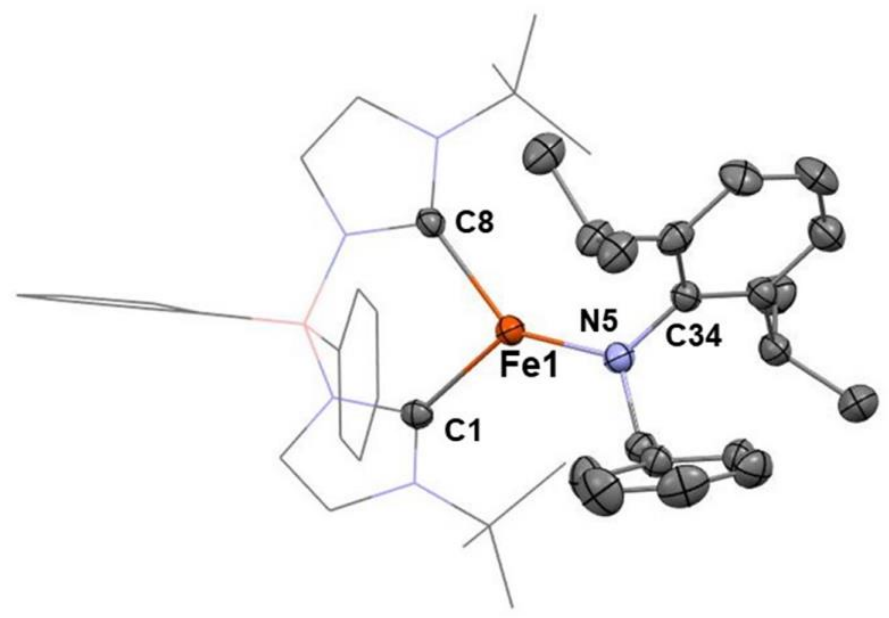

Figure S5. Molecular structure of $\left[\mathrm{Ph}{ }_{2} \mathrm{~B}\left({ }^{t} \mathrm{BuIm}\right){ }_{2} \mathrm{FeN}\left(\mathrm{CH}_{2} \mathrm{Ph}\right) \mathrm{Dipp}\right](7)$, as determined by single crystal X-ray diffraction. Ellipsoids are shown at 50\% probability level, bis(carbene)borate ligand (except for carbene carbon atoms) represented as wireframes. Solvent molecules and $\mathrm{H}$ atoms have been omitted for clarity. Color scheme: C, black; N, blue; B, pink; Fe, orange. Selected bond distances (Å) and angles (deg): Fe1-N5 1.901(3), Fe1-C1 2.096(3), Fe1-C8 2.081(3); C1-Fe1-C8 92.21(13), Fe1-N5-C34 125.8(2), C1-Fe1-N5 129.12(13), C8-Fe1-N5 138.40(12). 
NMR spectra

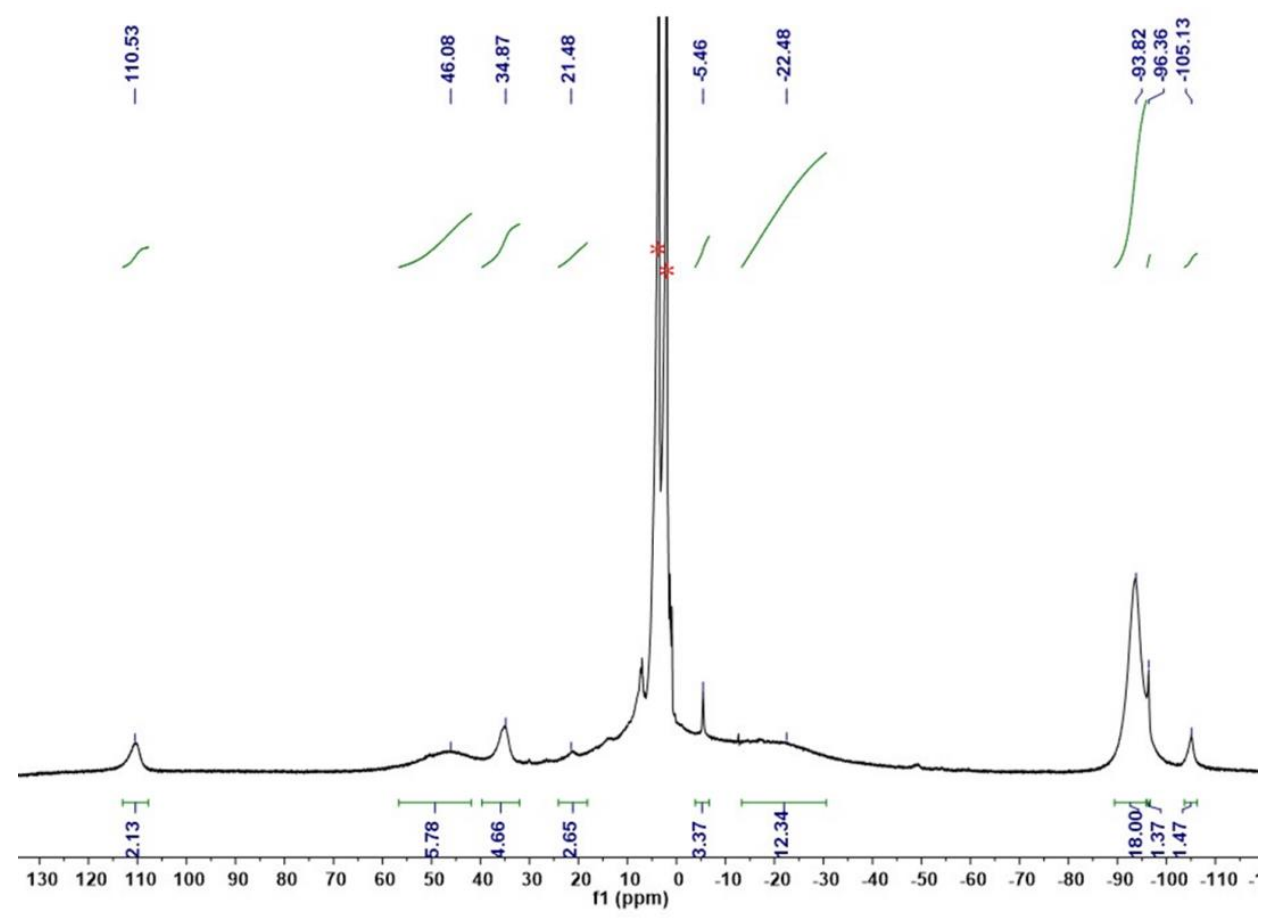

Figure S6. ${ }^{1} \mathrm{H}$ NMR spectrum $\left(400 \mathrm{MHz}, 25{ }^{\circ} \mathrm{C}, d_{8}\right.$ - THF $\left.\left({ }^{*}\right)\right)$ of $\left[\mathrm{Ph}_{2} \mathrm{~B}\left({ }^{t} \mathrm{BuIm}\right)_{2} \mathrm{FeNDippLi}(\mathrm{THF})_{2}\right]$ (2).

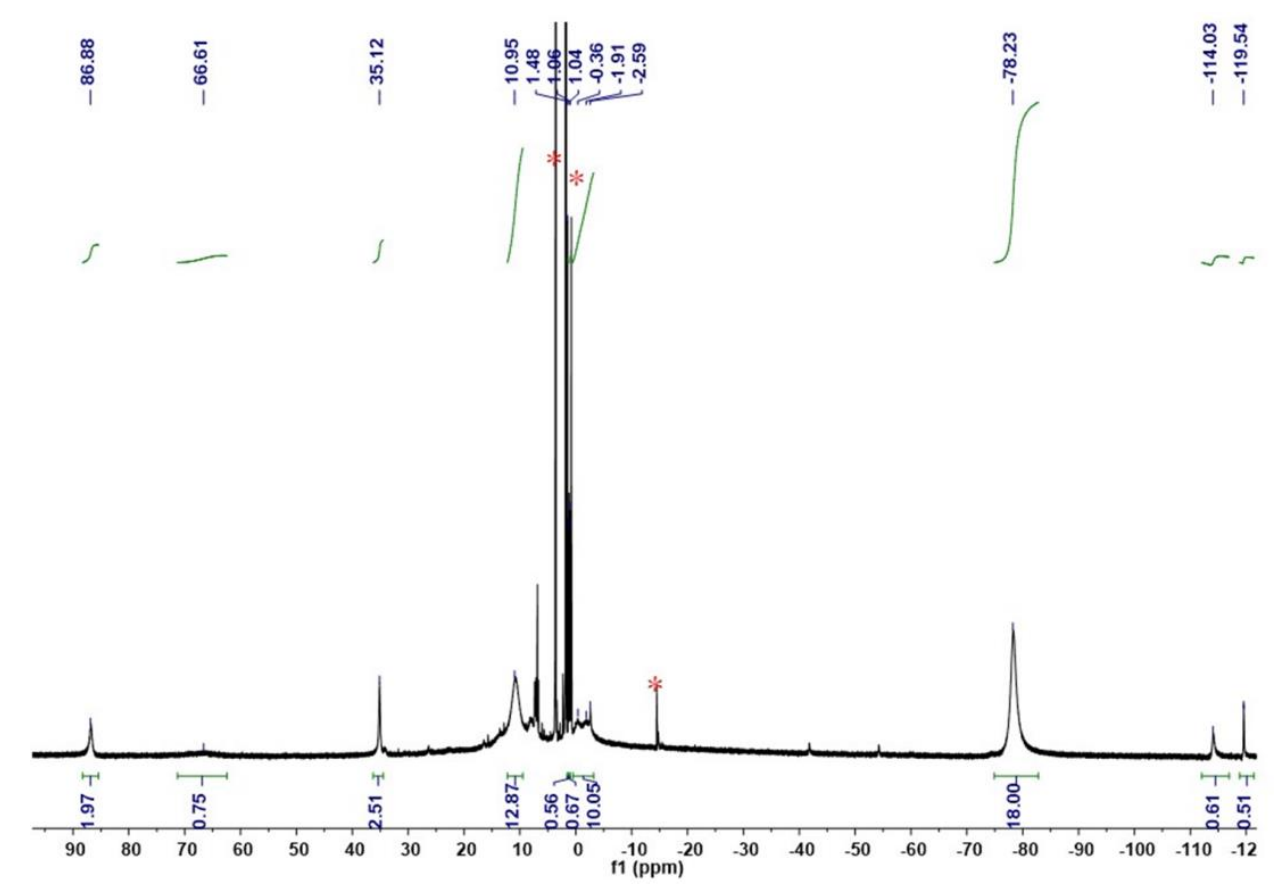

Figure S7. ${ }^{1} \mathrm{H}$ NMR spectrum $\left(400 \mathrm{MHz}, 25{ }^{\circ} \mathrm{C}, d_{8}\right.$-THF and instrumental artifact $\left.(*)\right)$ of $\left[\mathrm{Ph}_{2} \mathrm{~B}\left({ }^{t} \mathrm{BuIm}\right)_{2} \mathrm{FeNDippNa}(\mathrm{THF})_{3}\right](3)$. 


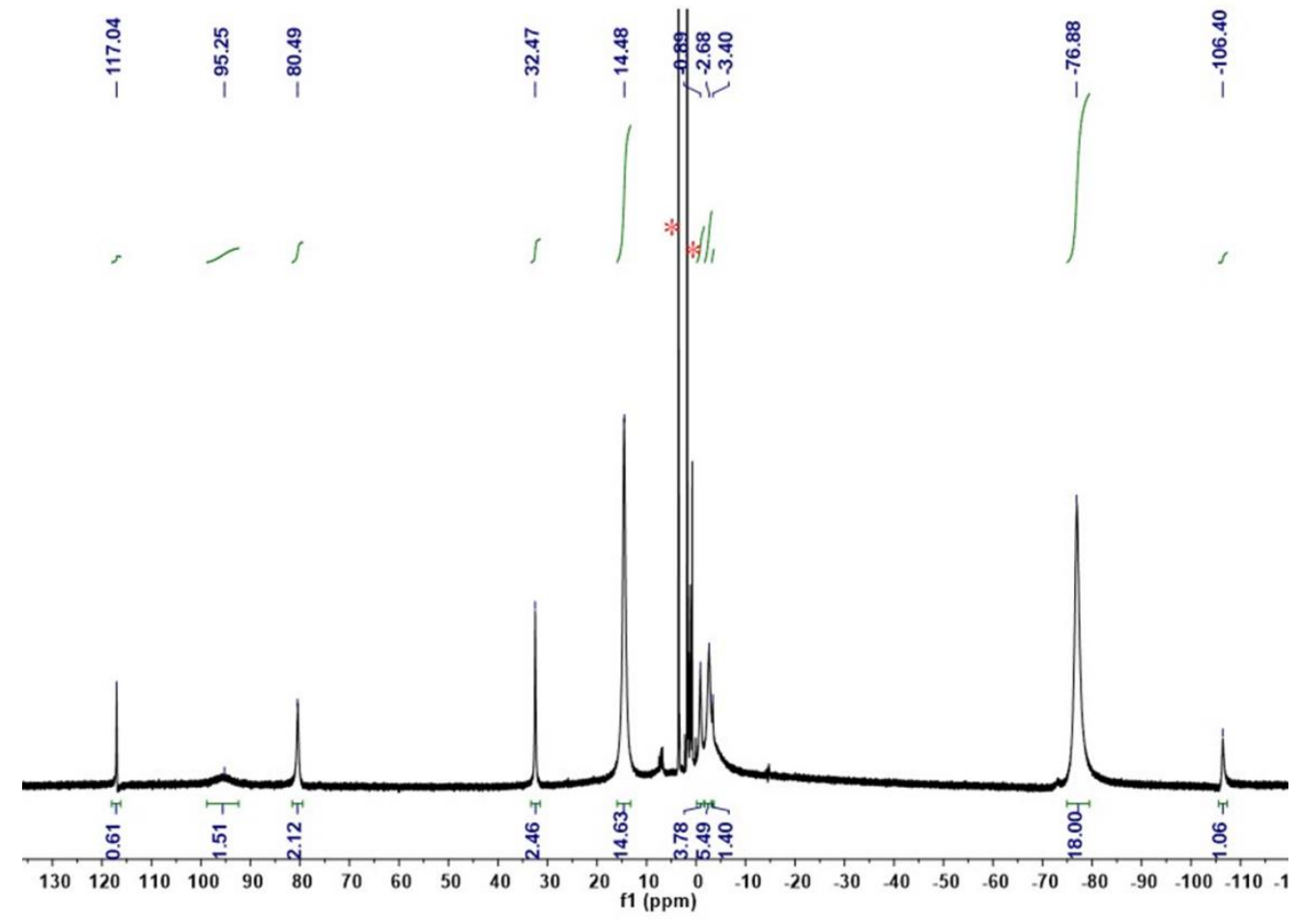

Figure S8. ${ }^{1} \mathrm{H}$ NMR spectrum $\left(400 \mathrm{MHz}, 25^{\circ} \mathrm{C}, d_{8}\right.$ - THF $\left.(*)\right)$ of $\left[\mathrm{Ph}_{2} \mathrm{~B}\left({ }^{t} \mathrm{BuIm}\right)_{2} \mathrm{FeNDippK}\right]_{2}$ (4).

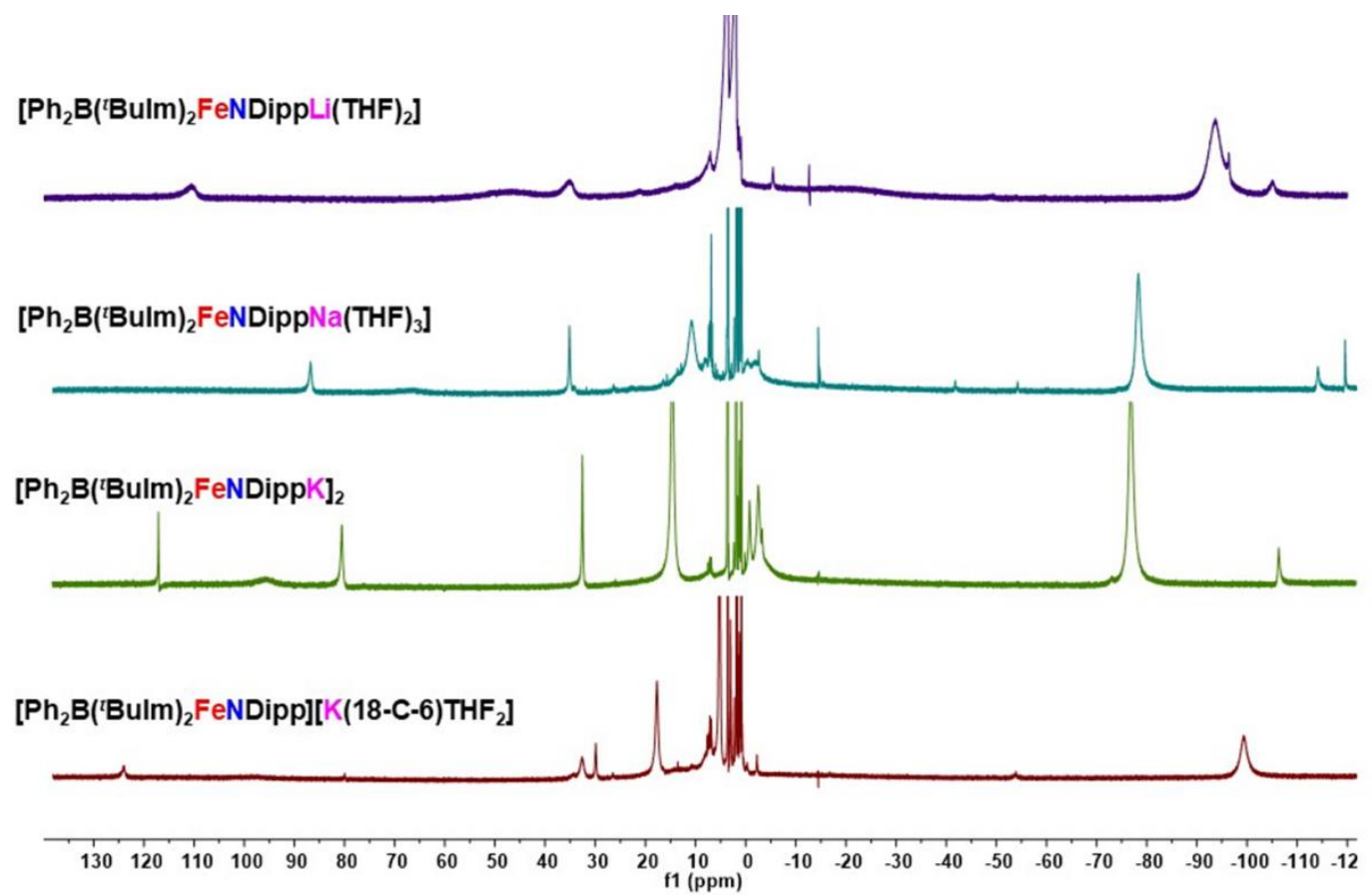

Figure S9. The comparision of ${ }^{1} \mathrm{H}$ NMR spectrum of $\left[\mathrm{Ph}_{2} \mathrm{~B}\left({ }^{t} \mathrm{BuIm}\right)_{2} \mathrm{FeNDippLi}(\mathrm{THF})_{2}\right]$ (2), $\left[\mathrm{Ph}_{2} \mathrm{~B}\left({ }^{t} \mathrm{BuIm}\right)_{2} \mathrm{FeNDippNa}(\mathrm{THF})_{3}\right]$ (3), $\left[\mathrm{Ph}{ }_{2} \mathrm{~B}\left({ }^{t} \mathrm{BuIm}\right)_{2} \mathrm{FeNDippK}\right]_{2}$ (4) and $\left[\mathrm{Ph}_{2} \mathrm{~B}\left({ }^{t} \mathrm{BuIm}\right)_{2} \mathrm{FeNDipp}\right]\left[\mathrm{K}(18-\mathrm{C}-6) \mathrm{THF}_{2}\right]$ (5) in THF- $d_{8}$. 


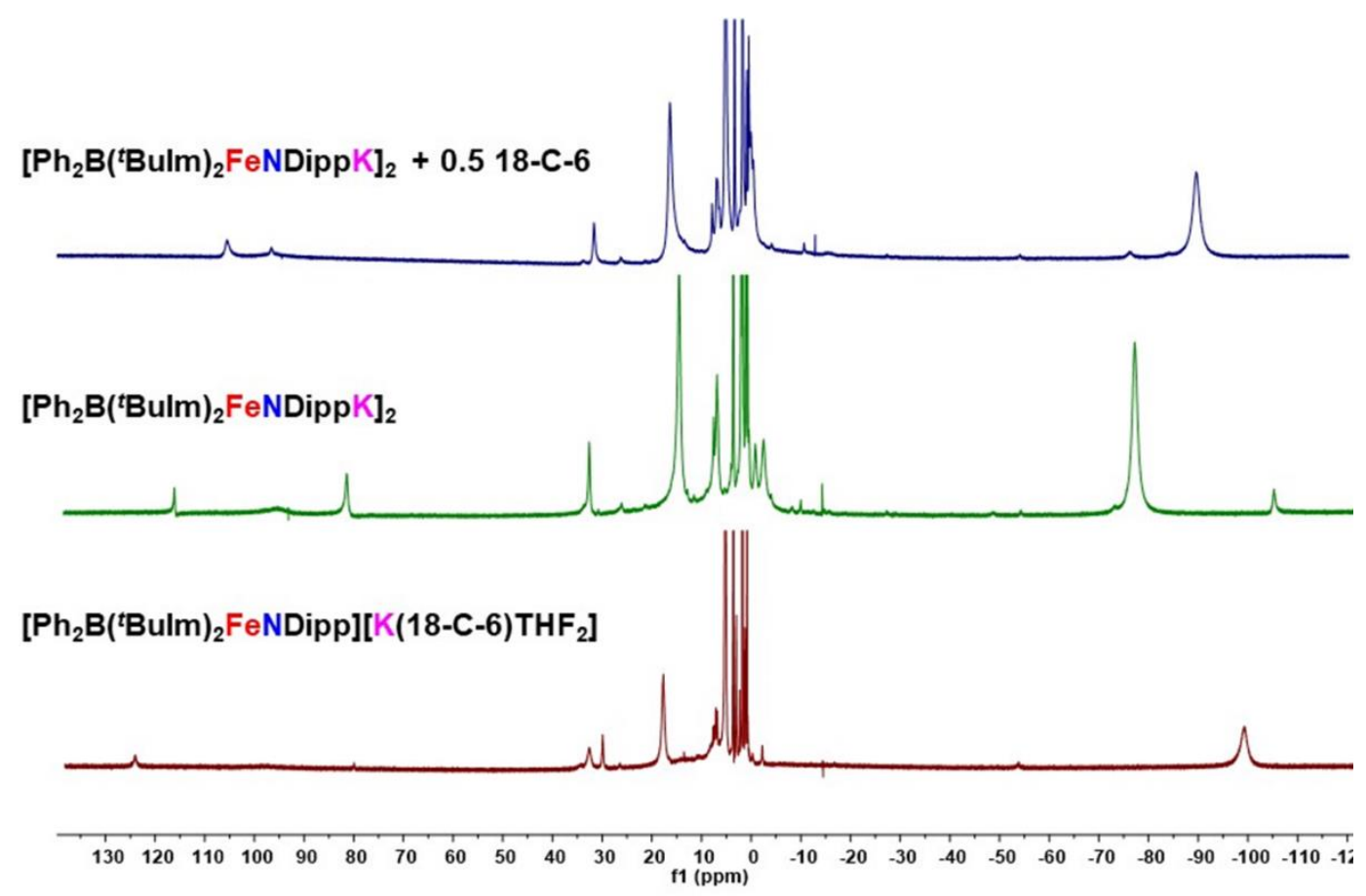

Figure S10. Comparison of ${ }^{1} \mathrm{H}$ NMR spectra for $\left[\mathrm{Ph}_{2} \mathrm{~B}\left({ }^{t} \mathrm{BuIm}\right)_{2} \mathrm{FeNDippK}\right]_{2}(4)+0.5$ eq. 18-C-6, $\left[\mathrm{Ph}_{2} \mathrm{~B}\left({ }^{t} \mathrm{BuIm}\right)_{2} \mathrm{FeNDippK}\right]_{2}$ (4) and $\left[\mathrm{Ph}_{2} \mathrm{~B}\left({ }^{t} \mathrm{BuIm}\right)_{2} \mathrm{FeNDipp}\right]\left[\mathrm{K}(18-\mathrm{C}-6) \mathrm{THF}_{2}\right]$ (5) in $\mathrm{THF}-d_{8}$, indicating that $\mathrm{K}^{+}$is fast on the NMR timescale.

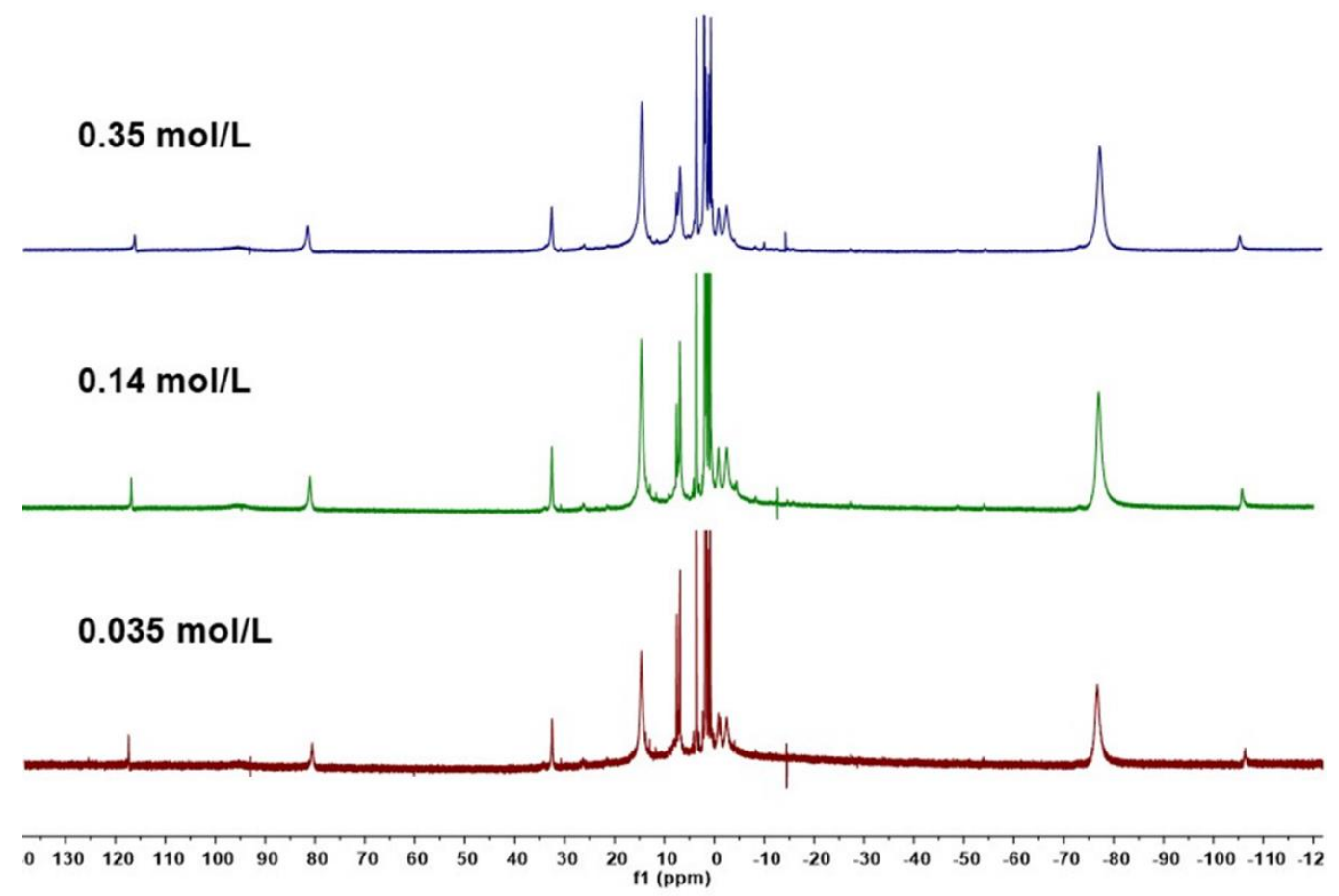

Figure S11. The ${ }^{1} \mathrm{H}$ NMR spectrum of $\left[\mathrm{Ph}_{2} \mathrm{~B}\left({ }^{t} \mathrm{BuIm}\right)_{2} \mathrm{FeNDippK}\right]_{2}$ (4) in THF- $d_{8}$ with different concentrations. The spectral invariance reveals that there are no equilibria involving $\mathrm{K}^{+}$exchange. 


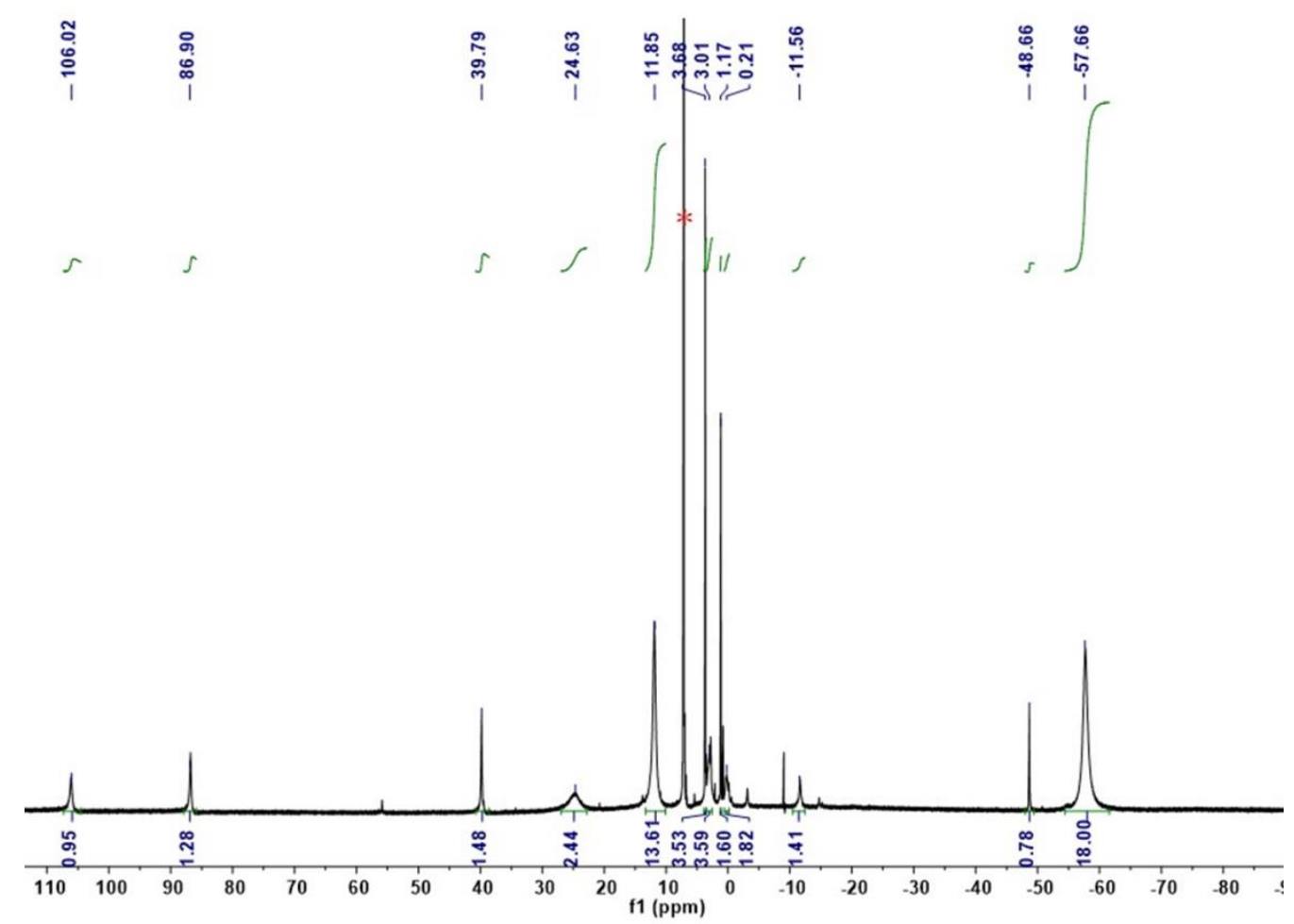

Figure S12. ${ }^{1} \mathrm{H}$ NMR spectrum $\left(400 \mathrm{MHz}, 25{ }^{\circ} \mathrm{C}, \mathrm{C}_{6} \mathrm{D}_{6}(*)\right)$ of $\left[\mathrm{Ph}_{2} \mathrm{~B}\left({ }^{t} \mathrm{BuIm}\right)_{2} \mathrm{FeNHDipp}\right](\mathbf{6})$.

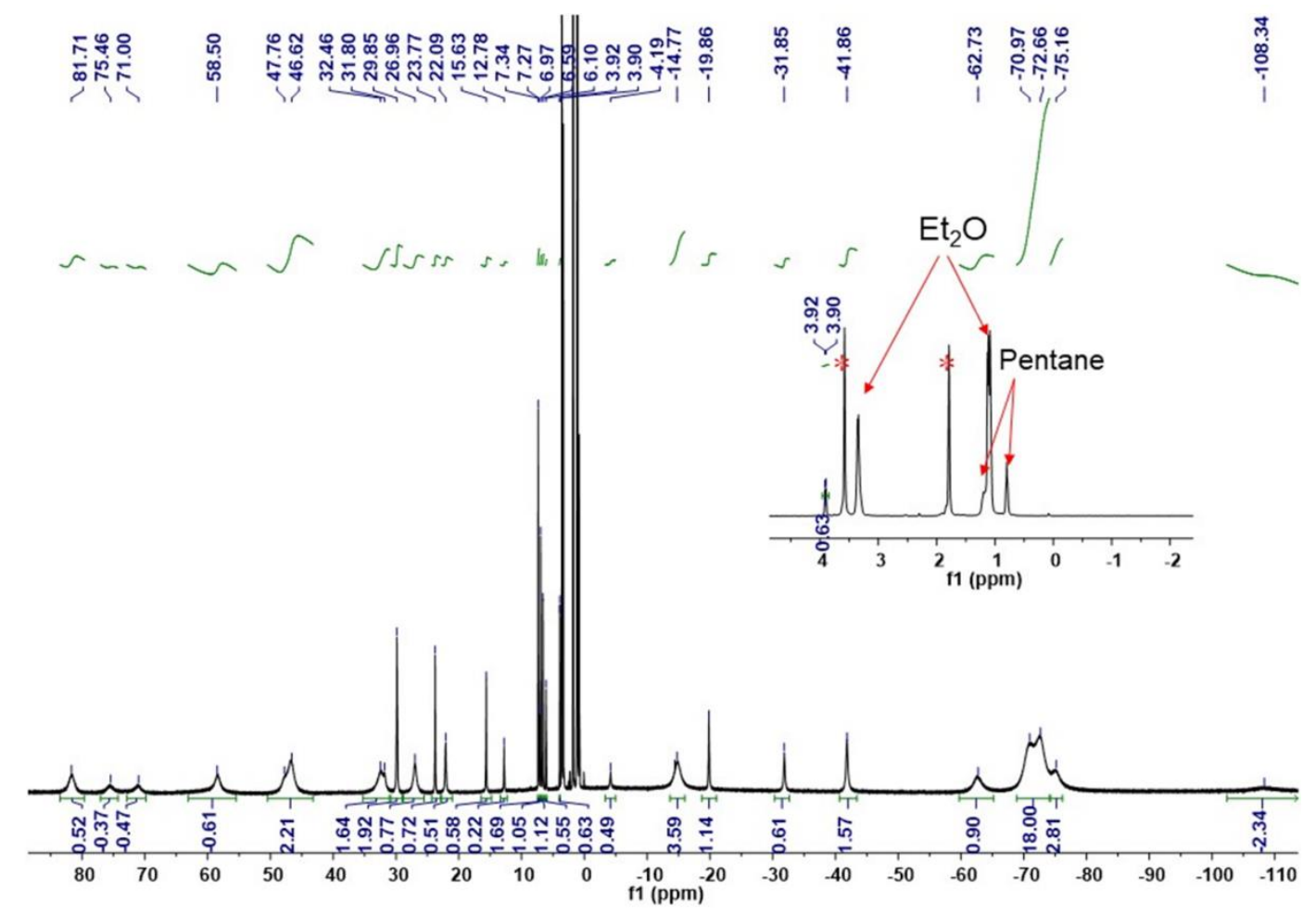

$\begin{array}{lllllllllll}\text { Figure } & \text { S13. } & { }^{1} \mathrm{H} & \mathrm{NMR} & \text { spectrum } & (400 & \mathrm{MHz}, & 25 & { }^{\circ} \mathrm{C}, & \mathrm{THF}-d_{8} & (*))\end{array}$ $\left[\mathrm{Ph}_{2} \mathrm{~B}\left({ }^{t} \mathrm{BuIm}\right)_{2} \mathrm{FeN}\left(\mathrm{CH}_{2} \mathrm{Ph}\right) \mathrm{Dipp}\right](7)$. 


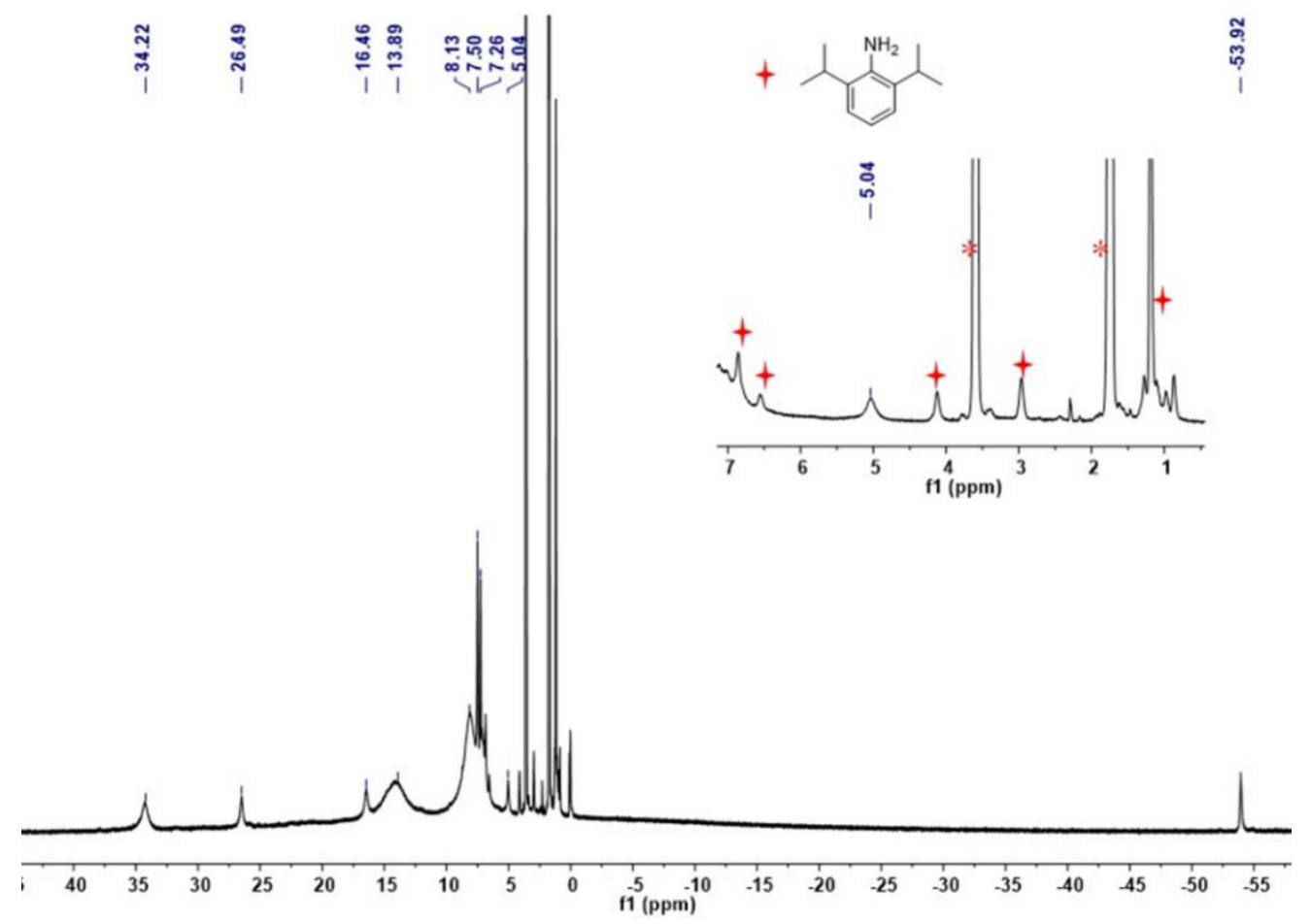

Figure S14. ${ }^{1} \mathrm{H}$ NMR spectrum $\left(400 \mathrm{MHz}, 25{ }^{\circ} \mathrm{C}, d_{8}-\mathrm{THF}\left({ }^{*}\right)\right)$ of $\left[\mathrm{Ph}{ }_{2} \mathrm{~B}\left({ }^{t} \mathrm{BuIm}\right)_{2} \mathrm{Fe}(\mathrm{NHDipp}){ }_{2} \mathrm{~K}\right]$ $(\mathbf{8}-\mathbf{K})$.

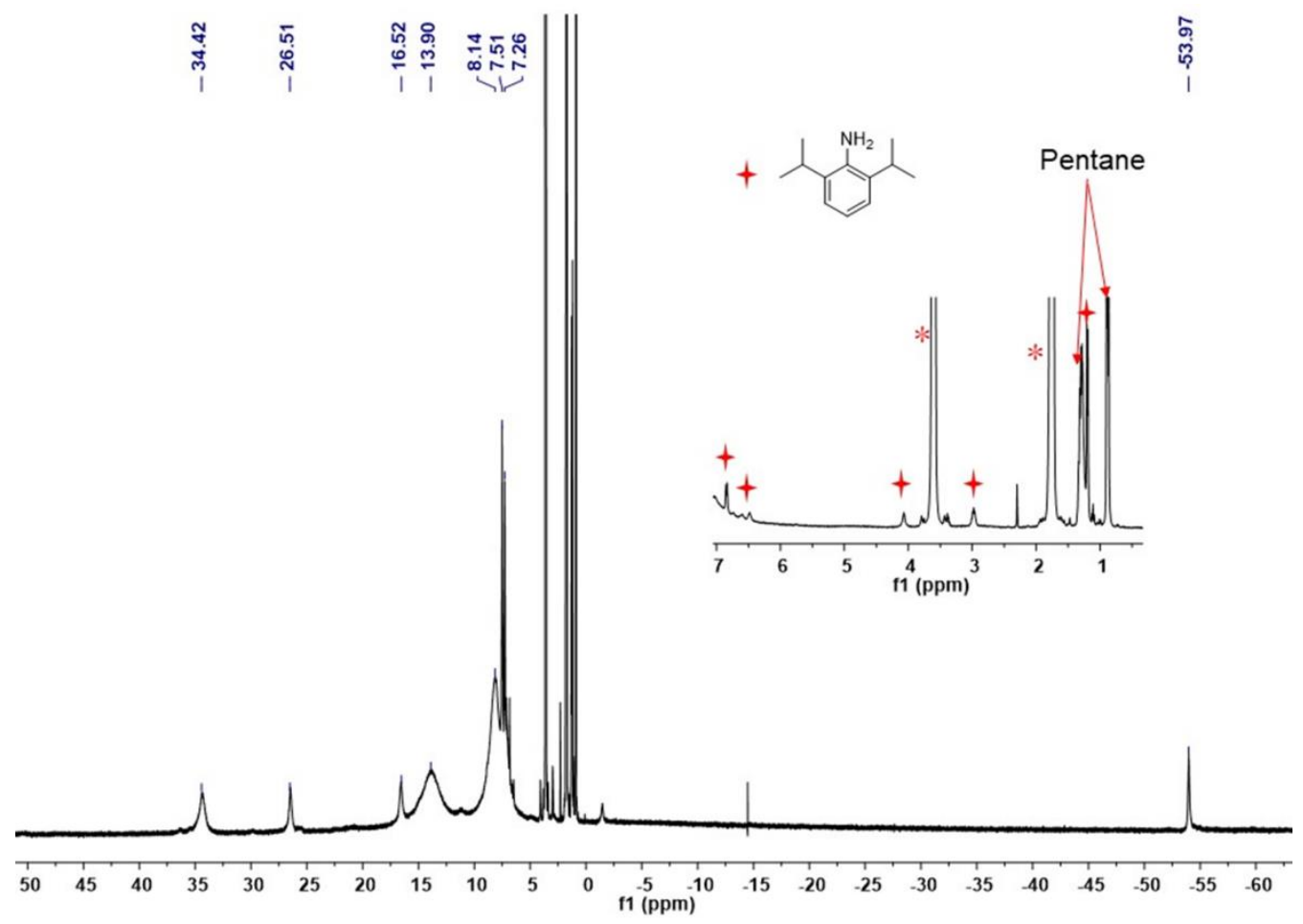

Figure S15. ${ }^{1} \mathrm{H}$ NMR spectrum $\left(400 \mathrm{MHz}, 25{ }^{\circ} \mathrm{C}, d_{8}-\mathrm{THF}(*)\right)$ of $\left[\mathrm{Ph}{ }_{2} \mathrm{~B}\left({ }^{t} \mathrm{BuIm}\right)_{2} \mathrm{Fe}(\mathrm{NHDipp}){ }_{2} \mathrm{Na}\right]$ (8-Na). 


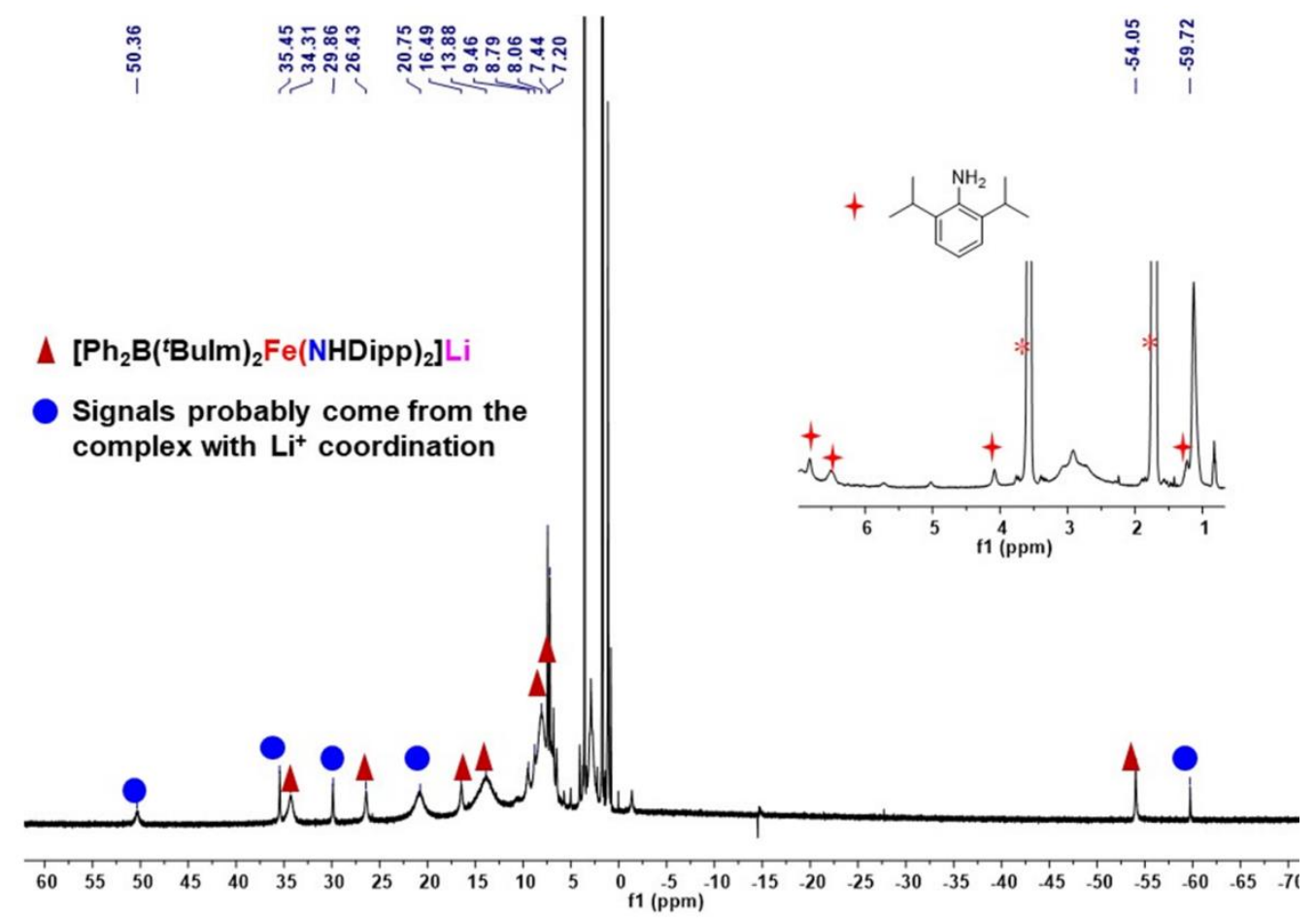

Figure S16. ${ }^{1} \mathrm{H}$ NMR spectrum $\left(400 \mathrm{MHz}, 25{ }^{\circ} \mathrm{C}\right.$, $d_{8}$-THF $\left.\left({ }^{*}\right)\right)$ of $\left[\mathrm{Ph}{ }_{2} \mathrm{~B}\left({ }^{t} \mathrm{BuIm}\right)_{2} \mathrm{Fe}(\mathrm{NHDipp})_{2} \mathrm{Li}\right]$ (8-Li).

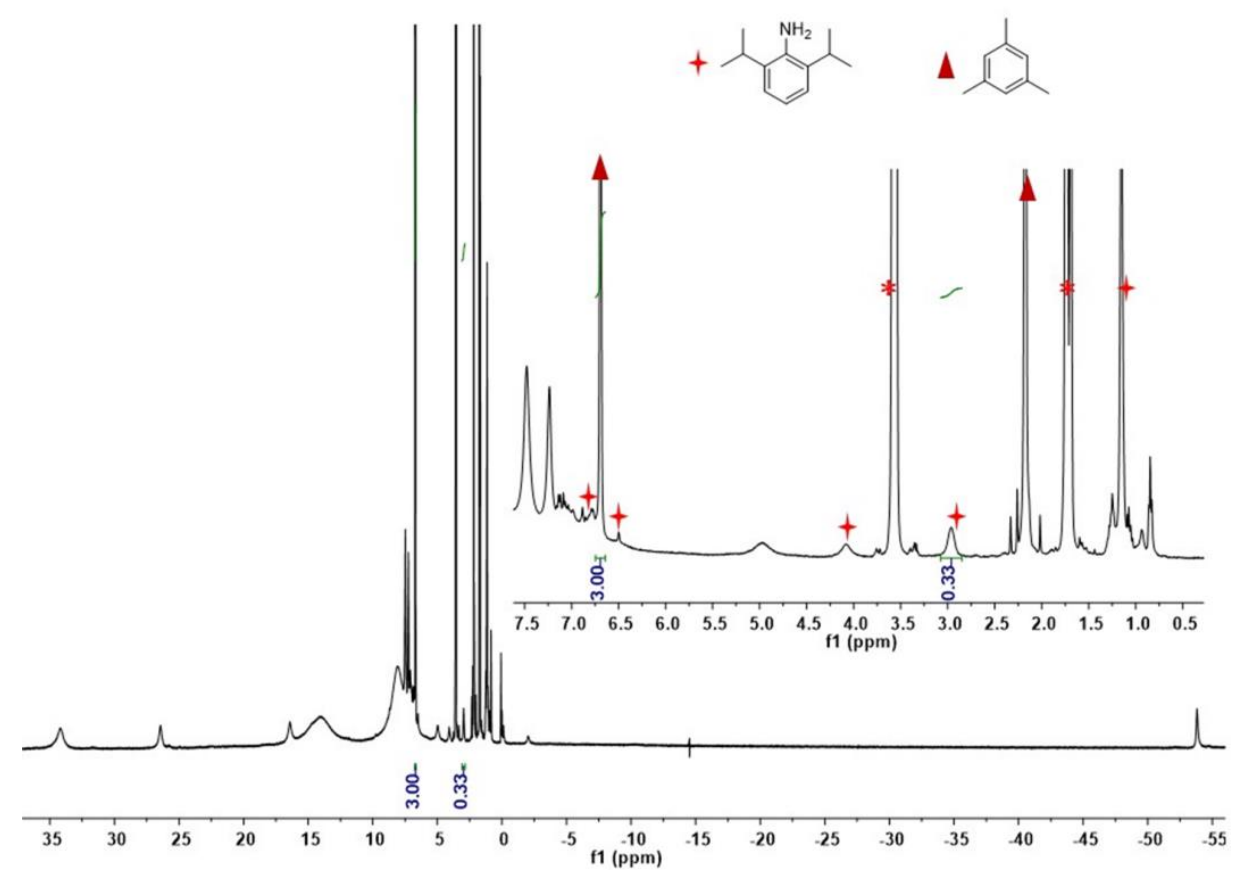

Figure S17. ${ }^{1} \mathrm{H}$ NMR spectrum $\left(400 \mathrm{MHz}, 25{ }^{\circ} \mathrm{C}, d_{8}\right.$-THF $\left.(*)\right)$ for equilibrium constant determination of $\left[\mathrm{Ph}_{2} \mathrm{~B}\left({ }^{t} \mathrm{BuIm}\right)_{2} \mathrm{Fe}(\mathrm{NHDipp})_{2} \mathrm{~K}\right](\mathbf{8}-\mathbf{K}) .0 .023 \mathrm{mmol}$ of $\mathbf{8}-\mathbf{K}$ and $0.042 \mathrm{mmol}$ of mesitylene were used. The calculated $K_{\text {eq }}=5.9 \times 10^{-3} \mathrm{~mol} / \mathrm{L}$. 


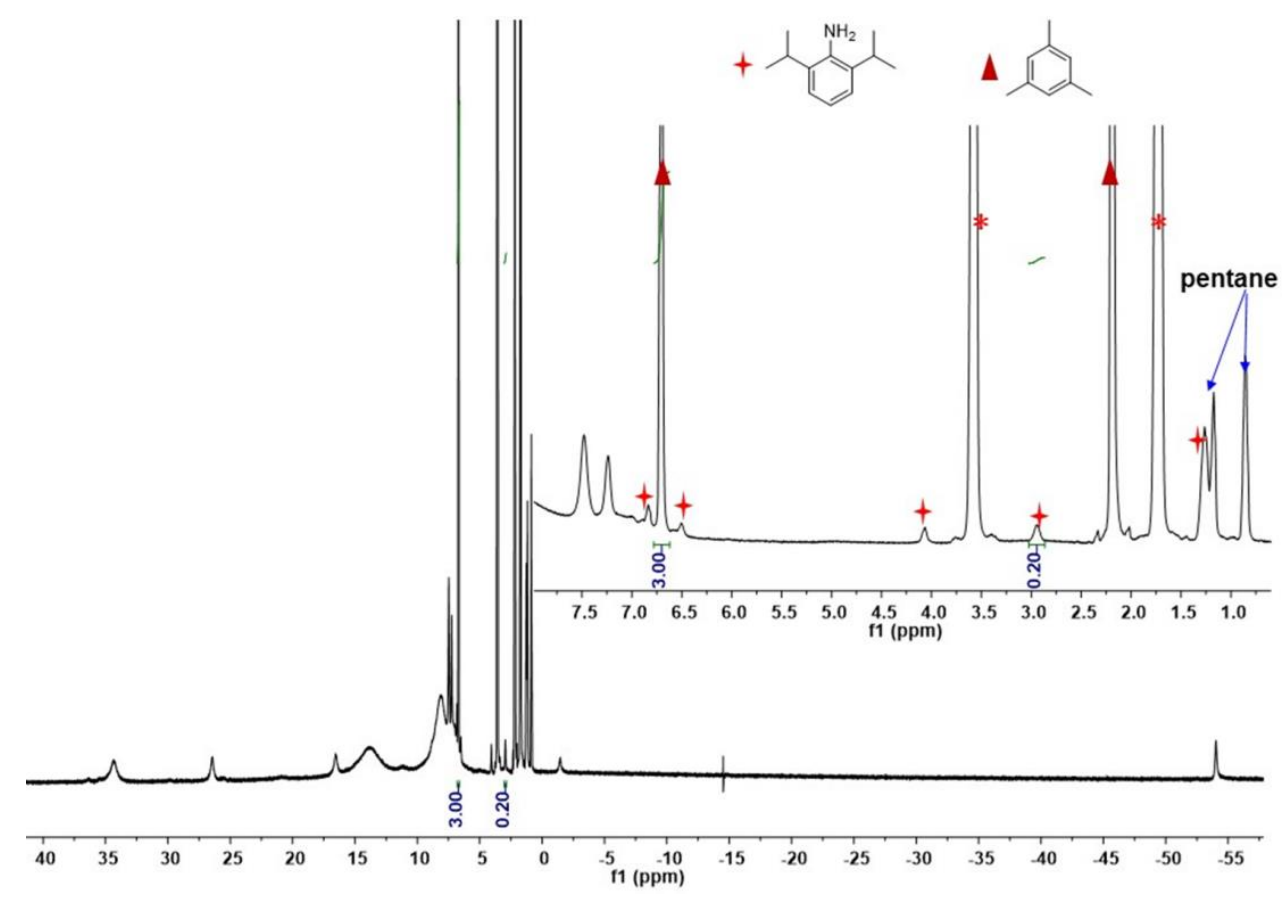

Figure S18. ${ }^{1} \mathrm{H}$ NMR spectrum $\left(400 \mathrm{MHz}, 25{ }^{\circ} \mathrm{C}, d_{8}\right.$-THF $\left.\left({ }^{*}\right)\right)$ for equilibrium constant determination of $\left[\mathrm{Ph}_{2} \mathrm{~B}\left({ }^{t} \mathrm{BuIm}\right)_{2} \mathrm{Fe}(\mathrm{NHDipp})_{2} \mathrm{Na}\right](\mathbf{8}-\mathrm{Na}) .0 .023 \mathrm{mmol}$ of 8-Na and $0.05 \mathrm{mmol}$ of mesitylene were used. The calculated $K_{\text {eq }}=2.9 \times 10^{-3} \mathrm{~mol} / \mathrm{L}$.

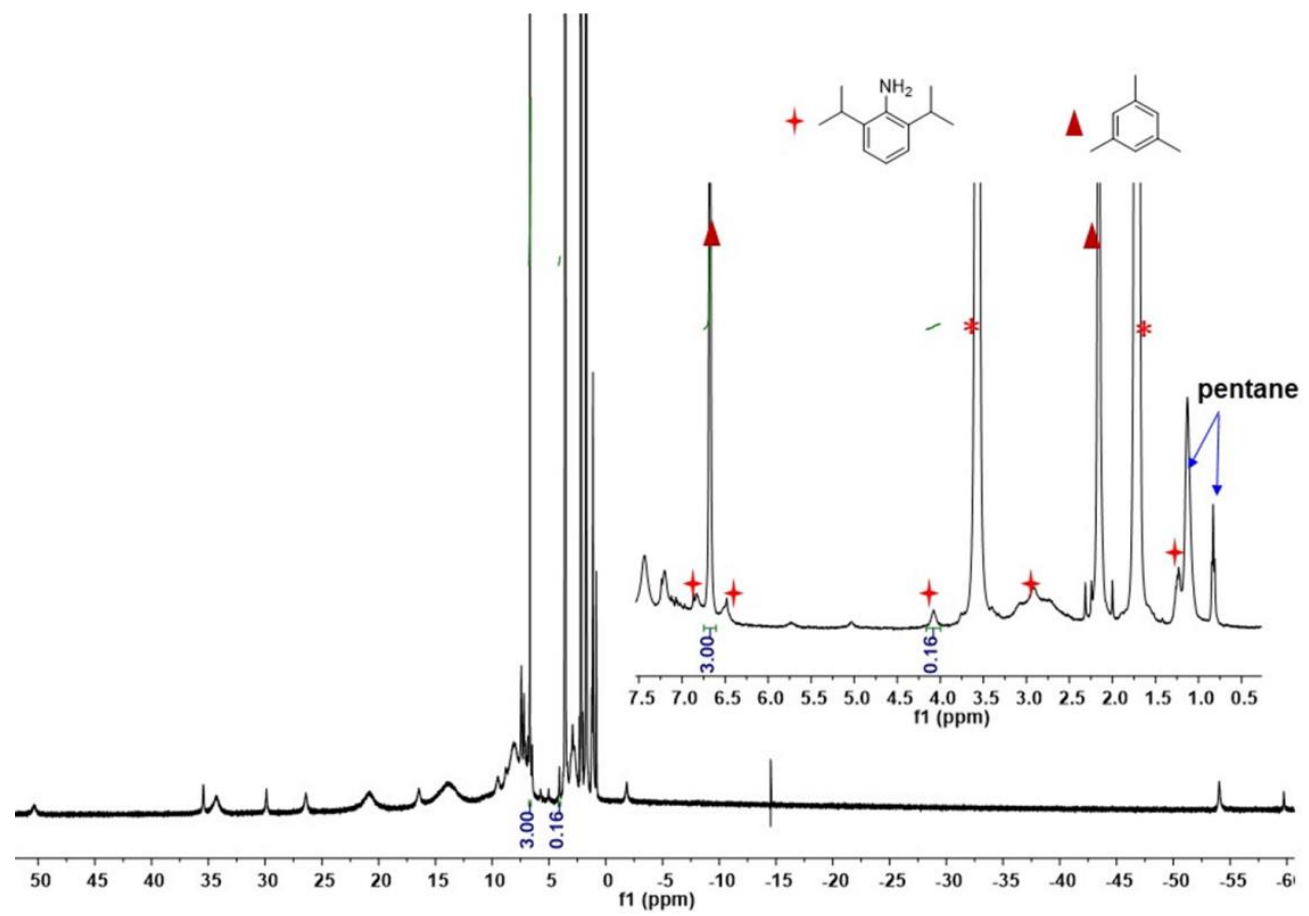

Figure S19. ${ }^{1} \mathrm{H}$ NMR spectrum $\left(400 \mathrm{MHz}, 25{ }^{\circ} \mathrm{C}, d_{8}\right.$-THF $\left.(*)\right)$ for equilibrium constant determination of $\left[\mathrm{Ph}_{2} \mathrm{~B}\left({ }^{t} \mathrm{BuIm}\right)_{2} \mathrm{Fe}(\mathrm{NHDipp})_{2} \mathrm{Li}\right]$ (8-Li). $0.023 \mathrm{mmol}$ of $\mathbf{8}-\mathbf{L i}$ and $0.05 \mathrm{mmol}$ of mesitylene were used. The calculated $K_{\text {eq }}=1.8 \times 10^{-3} \mathrm{~mol} / \mathrm{L}$. 


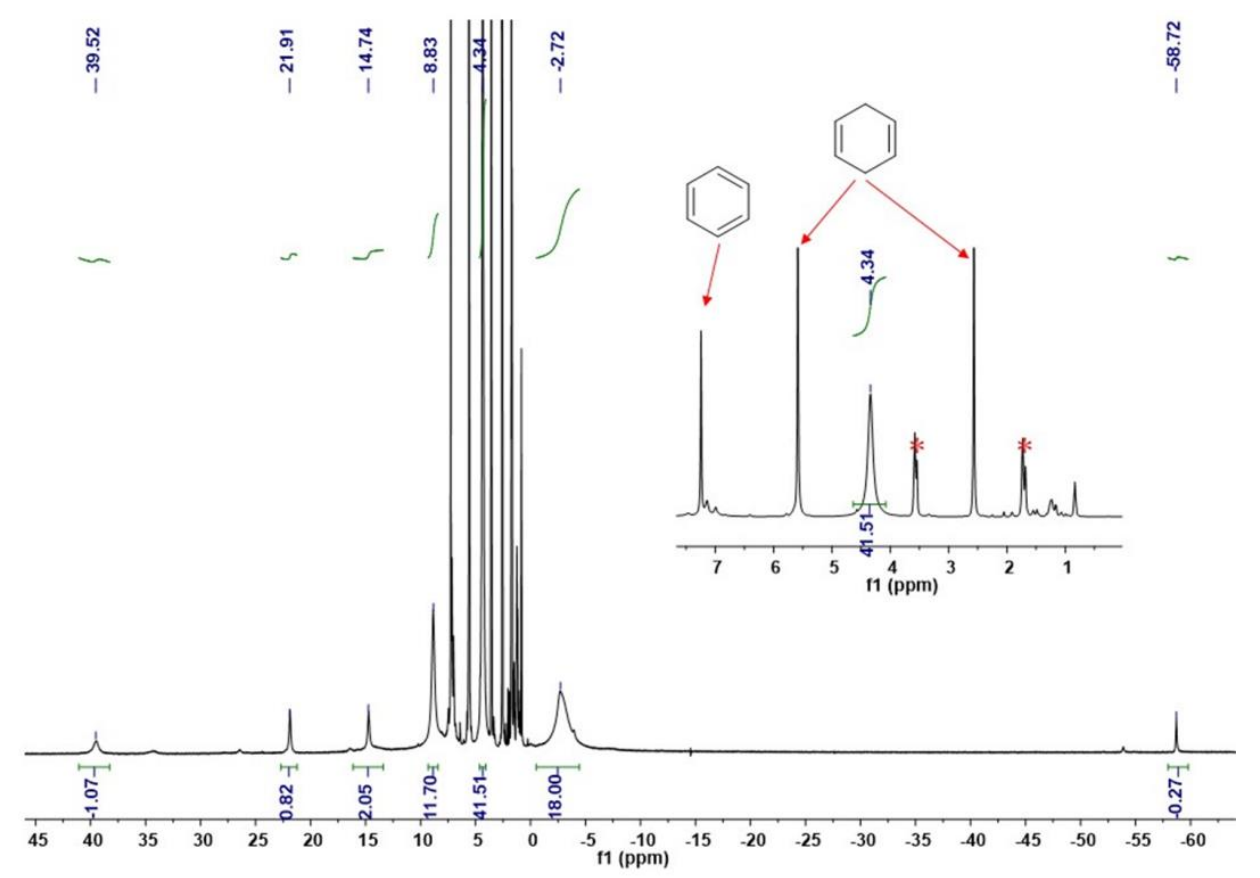

Figure S20. $\quad{ }^{1} \mathrm{H} \quad \mathrm{NMR}$ spectrum $\left(400 \quad \mathrm{MHz}, \quad 25 \quad{ }^{\circ} \mathrm{C}, \quad \mathrm{THF}-d_{8} \quad(*)\right) \quad$ of $\left[\mathrm{Ph}_{2} \mathrm{~B}\left({ }^{t} \mathrm{BuIm}\right)_{2} \mathrm{FeNHDipp}\right]\left[\mathrm{K}(18-\mathrm{C}-6) \mathrm{THF}_{2}\right](\mathbf{9}-[\mathbf{K}(\mathbf{1 8 - C}-\mathbf{6})])$ from the NMR tube reaction of $\left[\mathrm{Ph}_{2} \mathrm{~B}\left({ }^{t} \mathrm{BuIm}\right){ }_{2} \mathrm{FeNDipp}\right]\left[\mathrm{K}(18-\mathrm{C}-6) \mathrm{THF}_{2}\right](5)$ with 1,4-cyclohexadiene.

$+\left[\mathrm{Ph}_{2} \mathrm{~B}\left({ }^{\mathrm{t}} \mathrm{Bulm}\right)_{2} \mathrm{Fe}(\mathrm{NHDipp})_{2} \mathrm{~K}\right]$

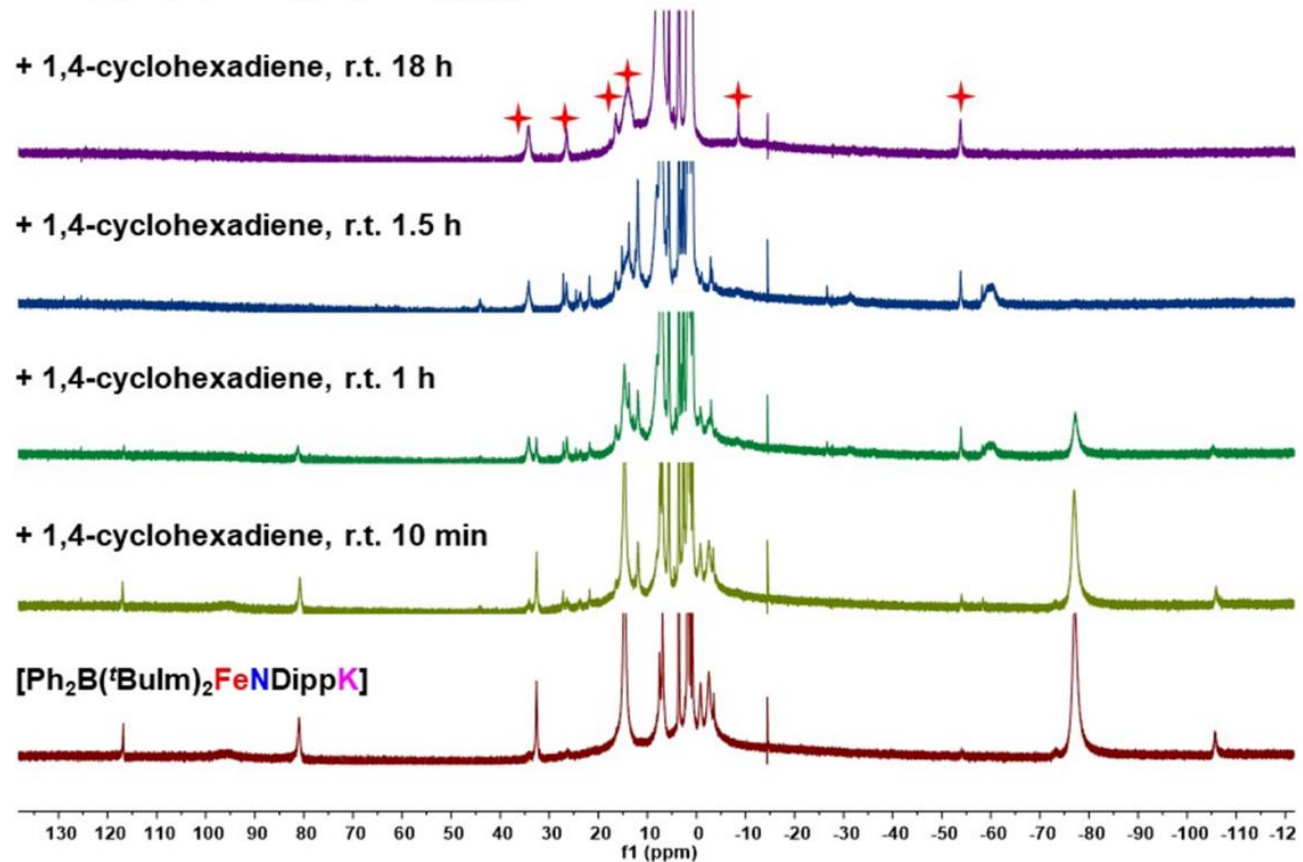

Figure S21. ${ }^{1} \mathrm{H}$ NMR spectrum $\left(400 \mathrm{MHz}, 25{ }^{\circ} \mathrm{C}, d_{8}\right.$-THF $)$ of the reaction of $\left[\mathrm{Ph}_{2} \mathrm{~B}\left({ }^{t} \mathrm{BuIm}\right)_{2} \mathrm{FeNDippK}\right]_{2}$ (4) with 1,4-cyclohexadiene. 
$+\left[\mathrm{Ph}_{2} \mathrm{~B}\left({ }^{\mathrm{t}} \mathrm{Bulm}\right)_{2} \mathrm{Fe}(\mathrm{NHDipp})_{2} \mathrm{Na}\right]$

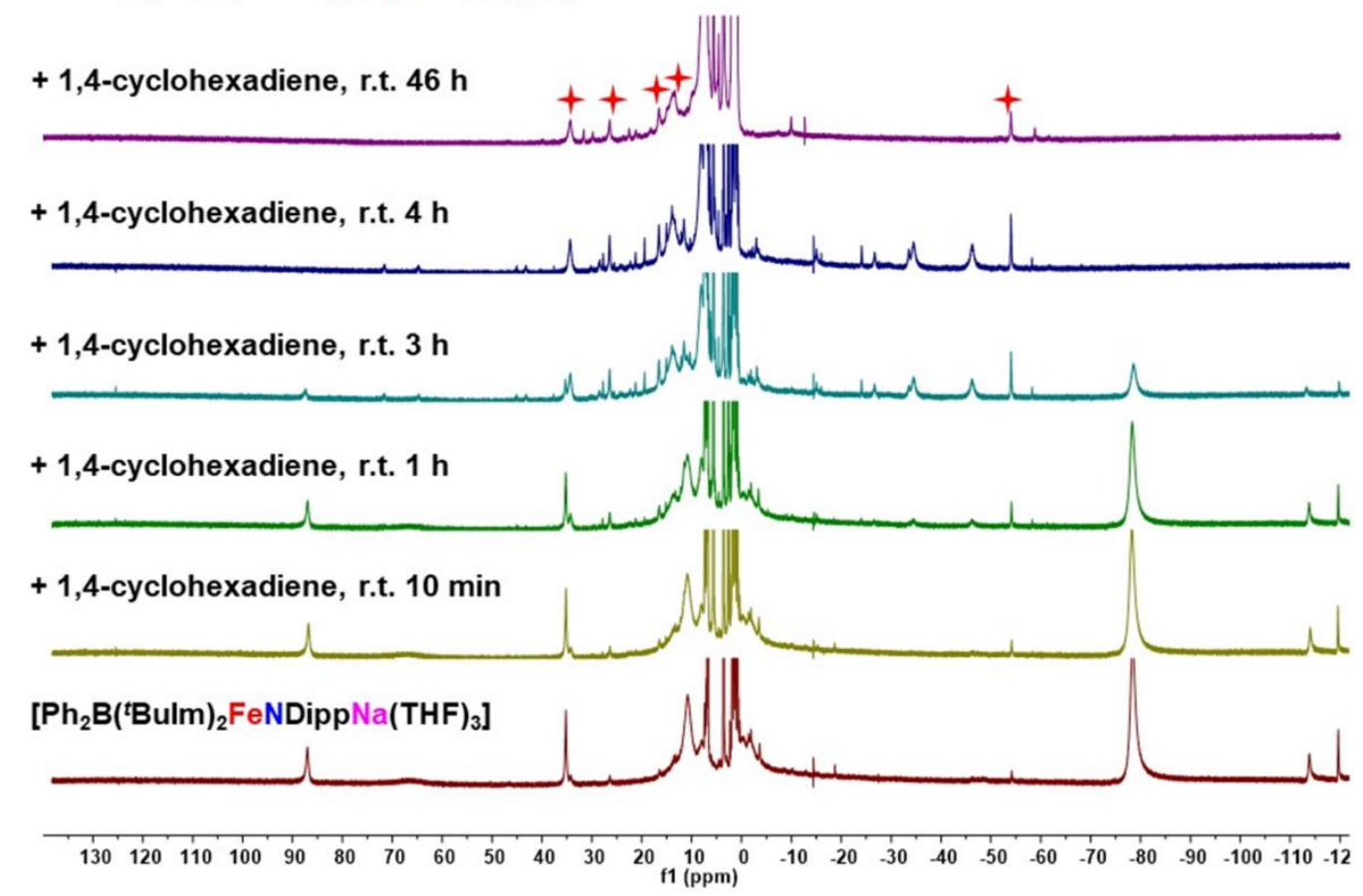

Figure S22. ${ }^{1} \mathrm{H}$ NMR spectrum $\left(400 \mathrm{MHz}, 25{ }^{\circ} \mathrm{C}, d_{8}-\mathrm{THF}\right)$ of the reaction of $\left[\mathrm{Ph}_{2} \mathrm{~B}\left({ }^{t} \mathrm{BuIm}\right)_{2} \mathrm{FeNDippNa}(\mathrm{THF})_{3}\right]$ (3) with 1,4-cyclohexadiene.

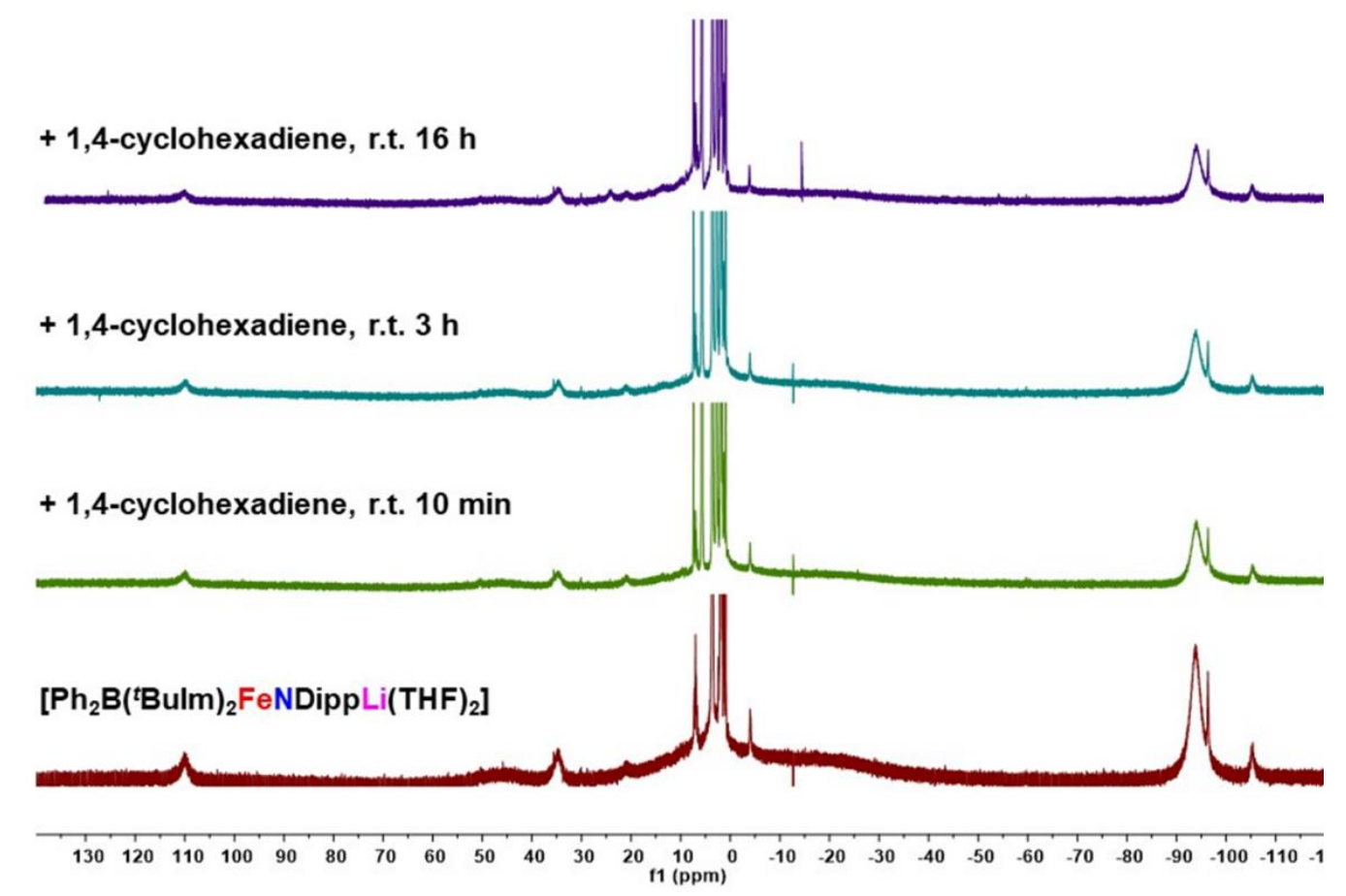

Figure S23. ${ }^{1} \mathrm{H}$ NMR spectrum $\left(400 \mathrm{MHz}, \quad 25{ }^{\circ} \mathrm{C}, d_{8}-\mathrm{THF}\right)$ of the reaction of $\left[\mathrm{Ph}_{2} \mathrm{~B}\left({ }^{t} \mathrm{BuIm}\right)_{2} \mathrm{FeNDippLi}(\mathrm{THF})_{2}\right](2)$ with 1,4-cyclohexadiene. 


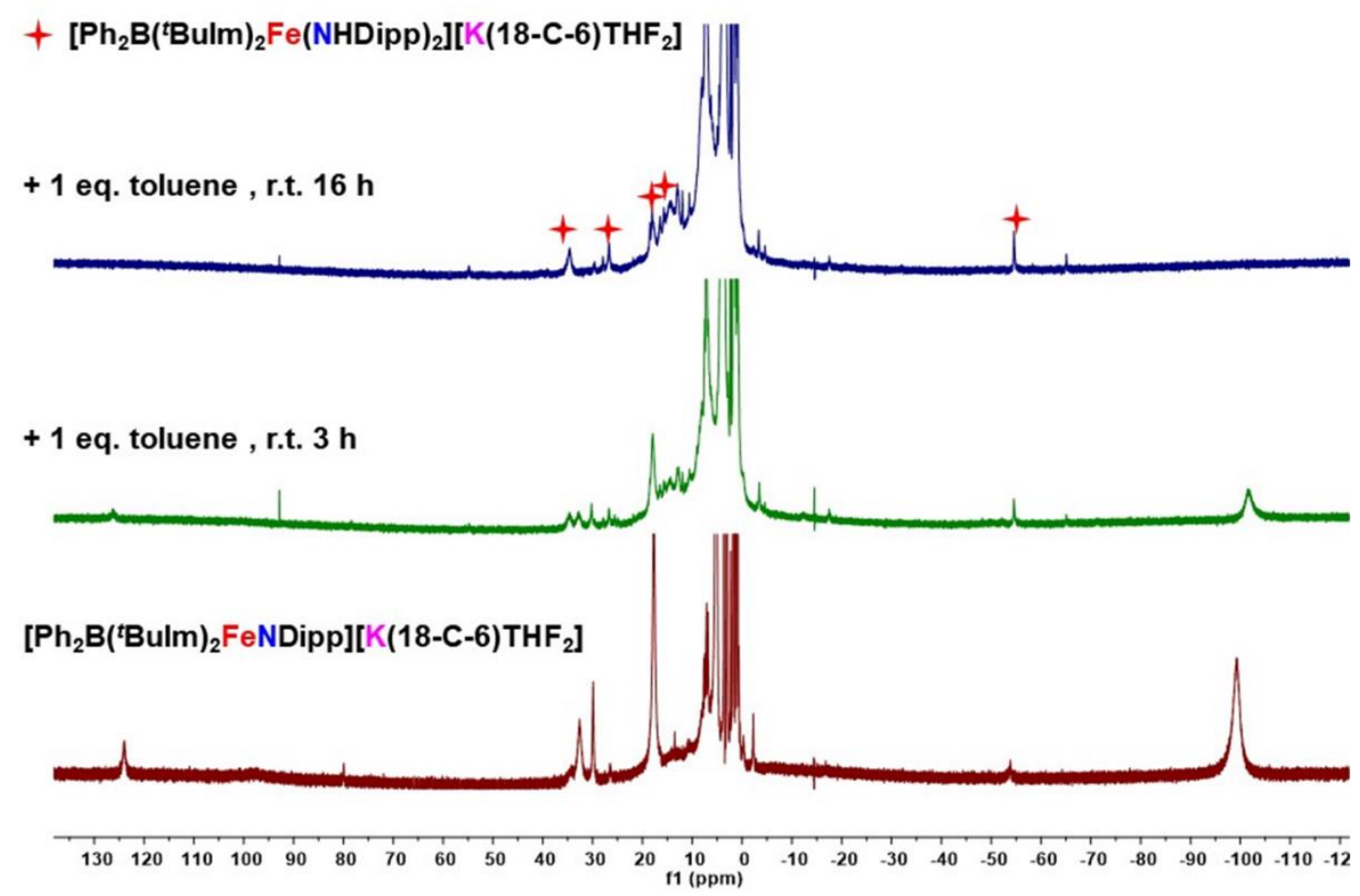

Figure S24. ${ }^{1} \mathrm{H}$ NMR spectrum (400 MHz, $25{ }^{\circ} \mathrm{C}, d_{8}$-THF) of the reaction of $\left[\mathrm{Ph}_{2} \mathrm{~B}\left({ }^{t} \mathrm{BuIm}\right)_{2} \mathrm{FeNDipp}\right]\left[\mathrm{K}(18-\mathrm{C}-6) \mathrm{THF}_{2}\right](5)$ with toluene.

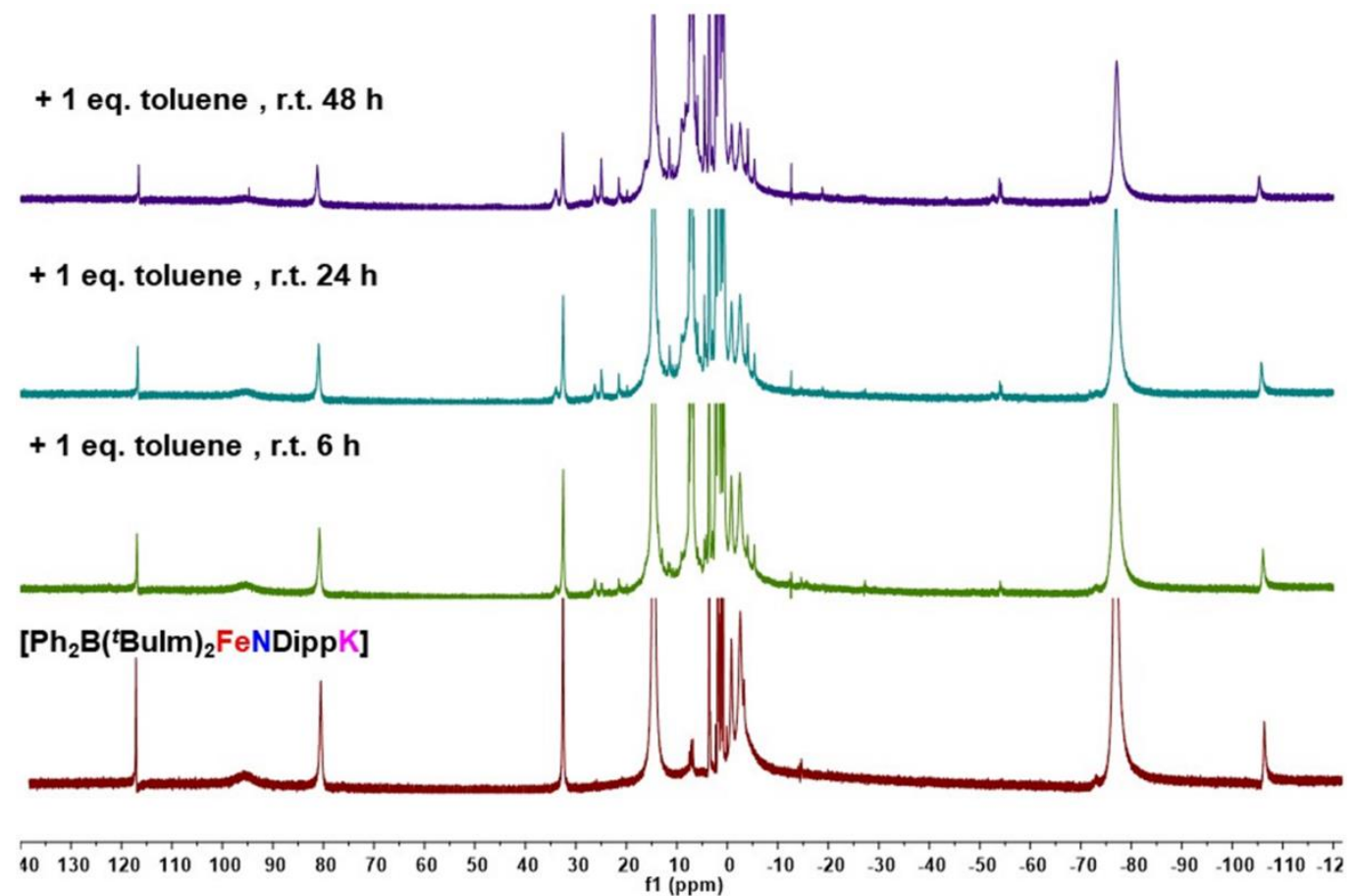

Figure S25. ${ }^{1} \mathrm{H}$ NMR spectrum $\left(400 \mathrm{MHz}, 25{ }^{\circ} \mathrm{C}, d_{8}\right.$-THF) of the reaction of $\left[\mathrm{Ph}_{2} \mathrm{~B}\left({ }^{t} \mathrm{BuIm}\right)_{2} \mathrm{FeNDippK}\right]_{2}$ (4) with toluene. 


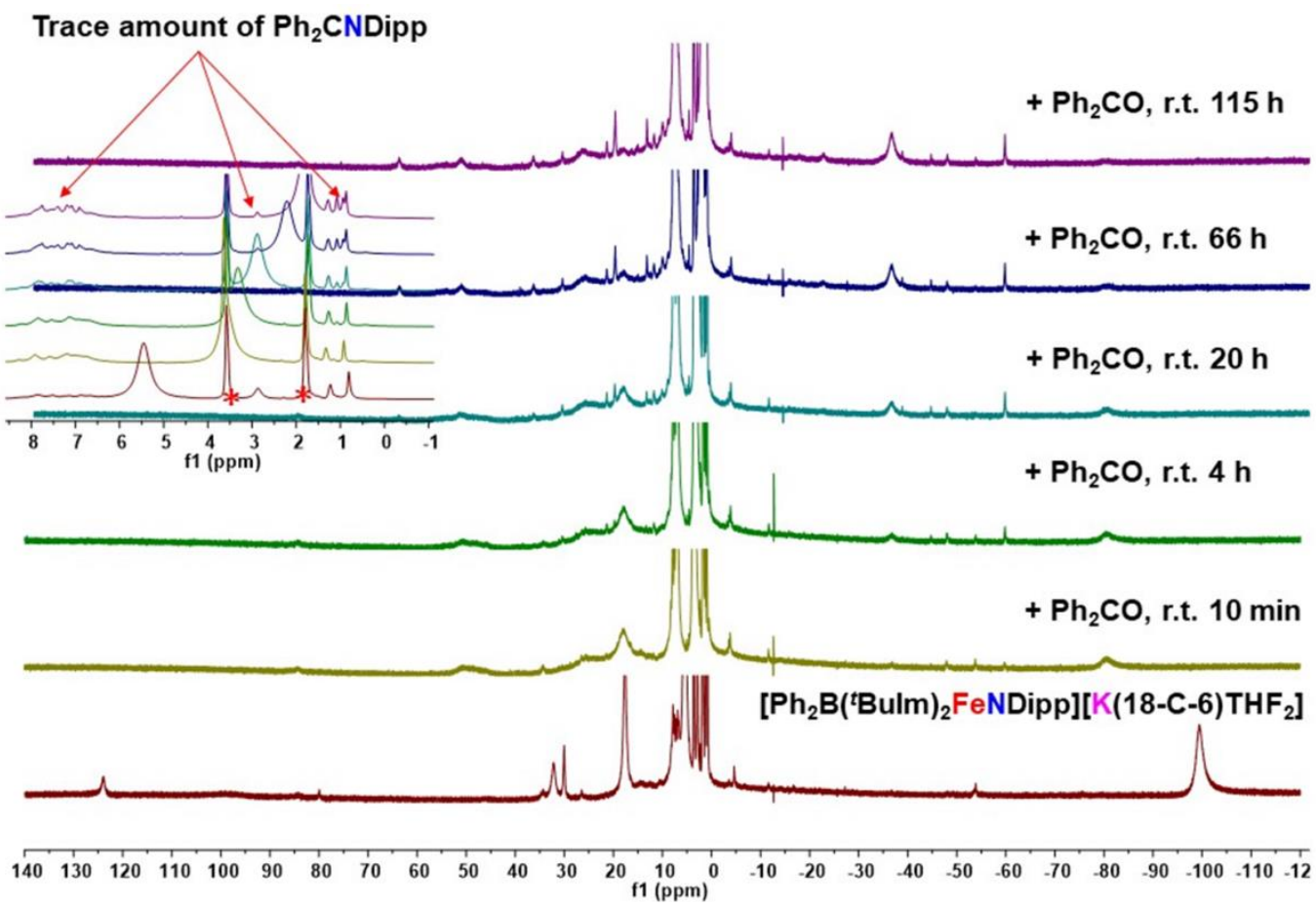

Figure S26. ${ }^{1} \mathrm{H}$ NMR spectrum (400 MHz, $25{ }^{\circ} \mathrm{C}$, THF- $\left.d_{8} \quad(*)\right)$ of the reaction of $\left[\mathrm{Ph}_{2} \mathrm{~B}\left({ }^{t} \mathrm{BuIm}\right)_{2} \mathrm{FeNDipp}\right]\left[\mathrm{K}(18-\mathrm{C}-6) \mathrm{THF}_{2}\right](5)$ with $\mathrm{Ph}_{2} \mathrm{CO}$.

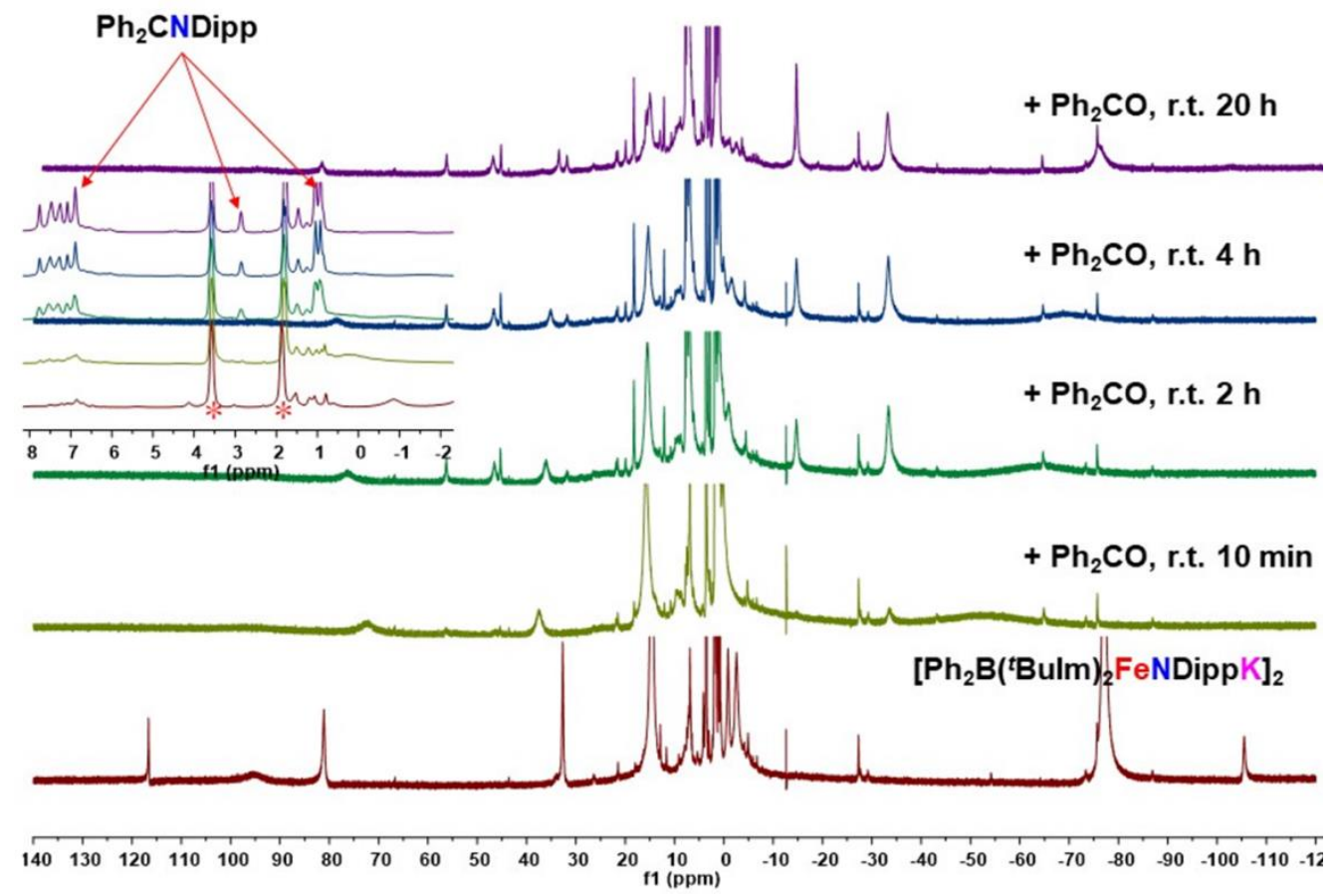

Figure S27. ${ }^{1} \mathrm{H}$ NMR spectrum $\left(400 \mathrm{MHz}, 25{ }^{\circ} \mathrm{C}\right.$, THF- $\left.d_{8}(*)\right)$ of the reaction of $\left[\mathrm{Ph}{ }_{2} \mathrm{~B}\left({ }^{t} \mathrm{BuIm}\right)_{2} \mathrm{FeNDippK}\right]_{2}$ (4) with $\mathrm{Ph}_{2} \mathrm{CO}$. 


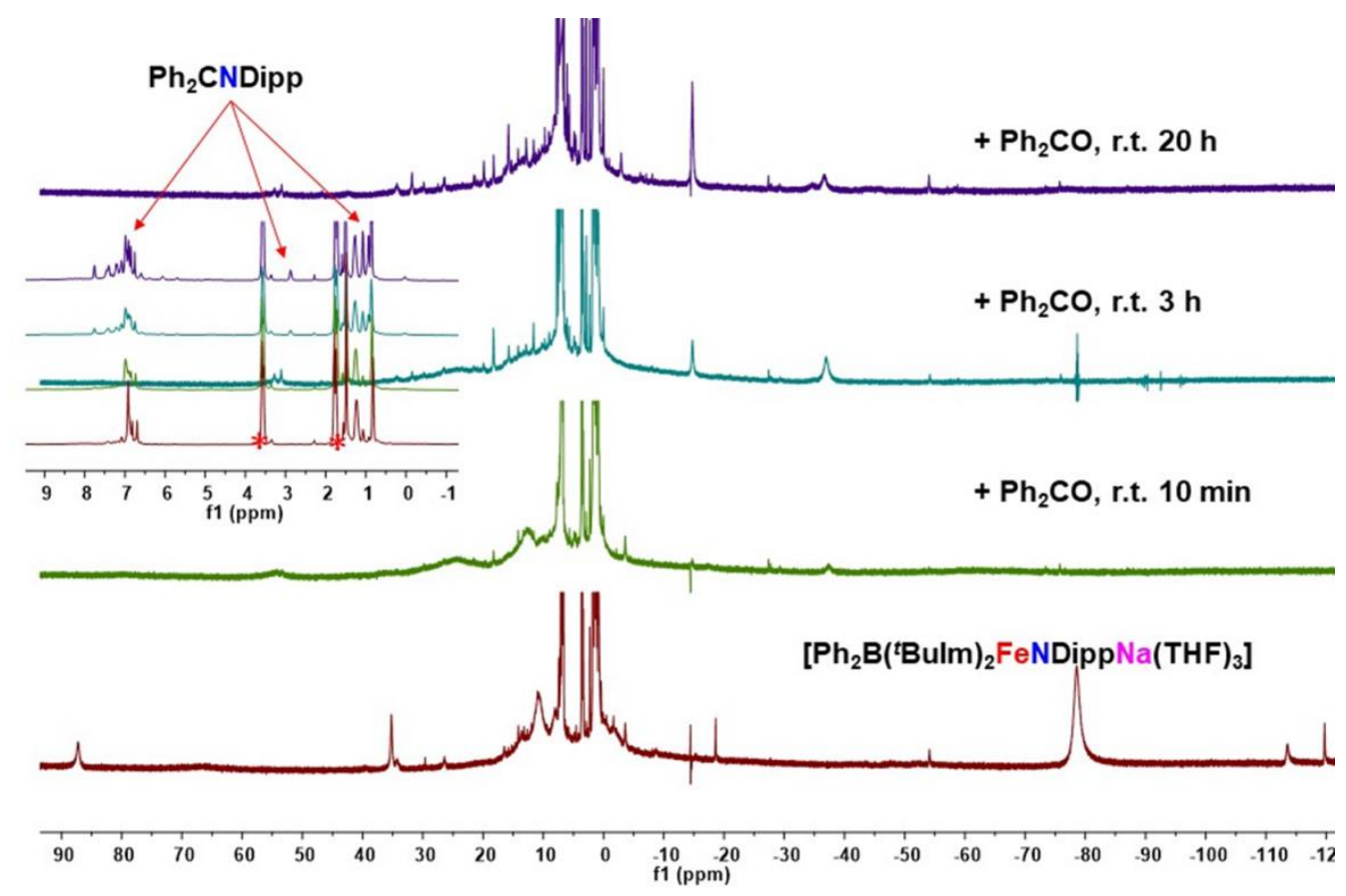

Figure S28. ${ }^{1} \mathrm{H}$ NMR spectrum $\left(400 \mathrm{MHz}, 25{ }^{\circ} \mathrm{C}\right.$, THF- $\left.d_{8}\left(^{*}\right)\right)$ of the reaction of $\left[\mathrm{Ph}_{2} \mathrm{~B}\left({ }^{t} \mathrm{BuIm}\right)_{2} \mathrm{FeNDippNa}(\mathrm{THF})_{2}\right](3)$ with $\mathrm{Ph}_{2} \mathrm{CO}$.

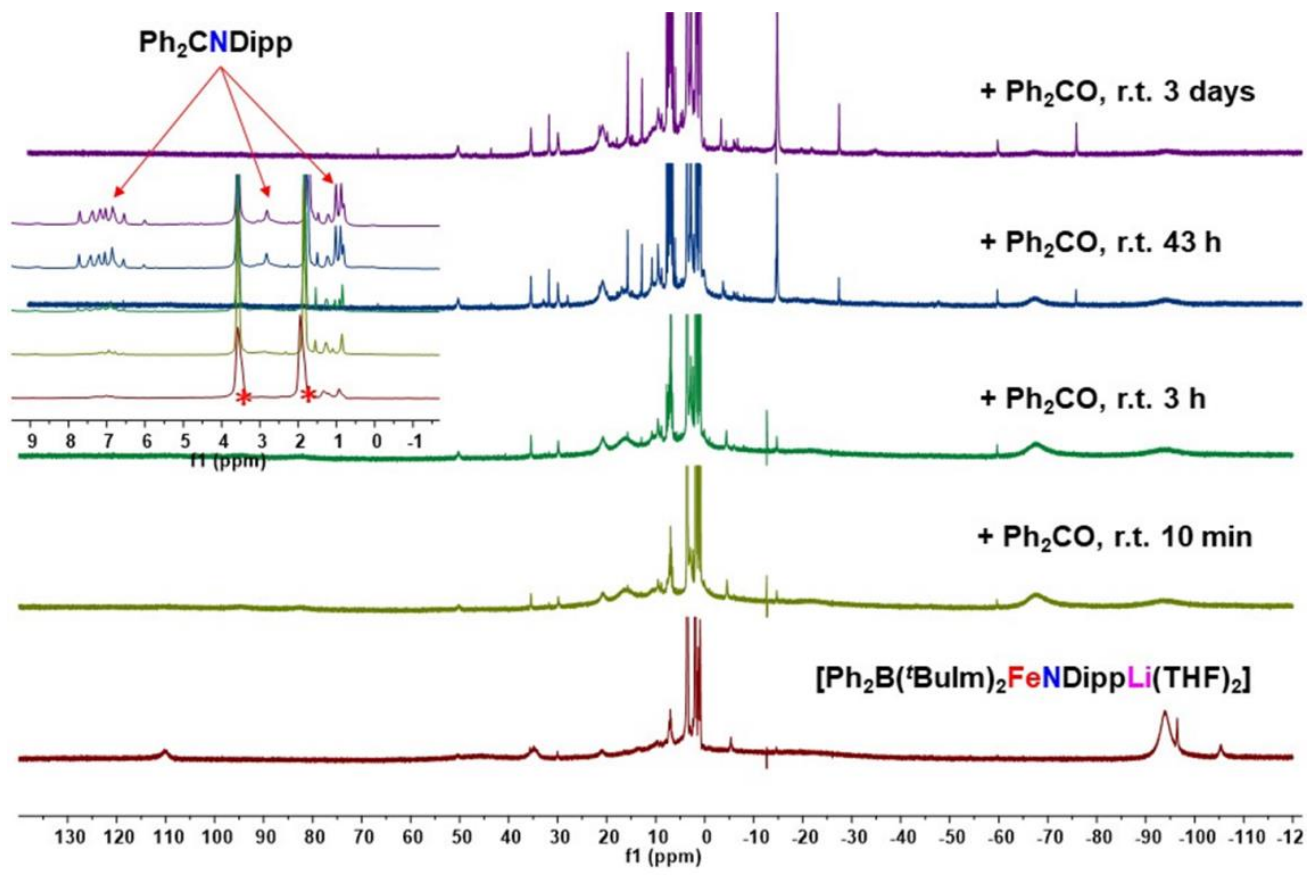

Figure S29. ${ }^{1} \mathrm{H}$ NMR spectrum $\left(400 \mathrm{MHz}, 25{ }^{\circ} \mathrm{C}\right.$, THF- $\left.d_{8}(*)\right)$ of the reaction of $\left[\mathrm{Ph}_{2} \mathrm{~B}\left({ }^{t} \mathrm{BuIm}\right)_{2} \mathrm{FeNDippLi}(\mathrm{THF})_{2}\right](2)$ with $\mathrm{Ph}_{2} \mathrm{CO}$. 


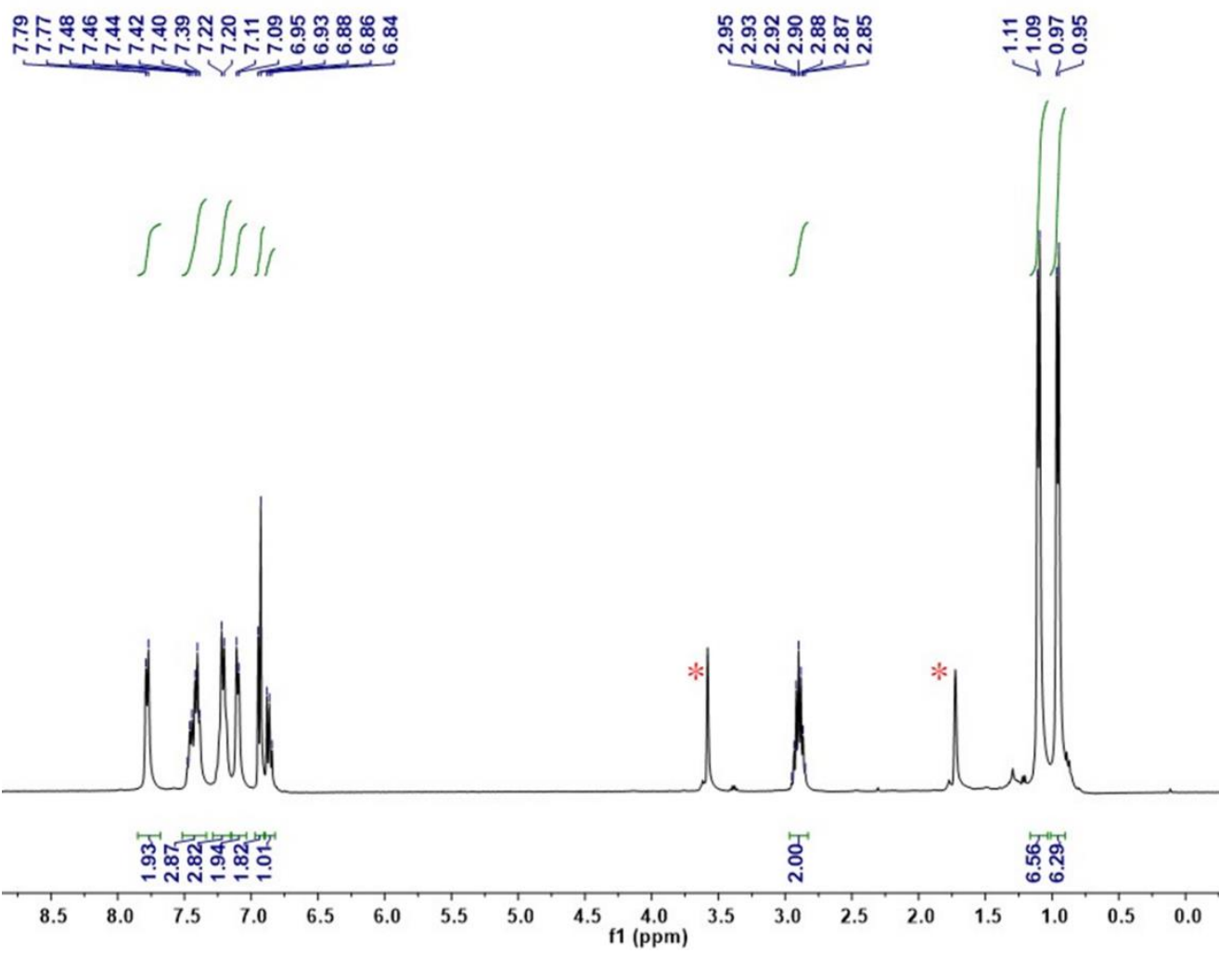

Figure S30. ${ }^{1} \mathrm{H}$ NMR spectrum $\left(400 \mathrm{MHz}, 25^{\circ} \mathrm{C}\right.$, THF- $\left.d_{8}(*)\right)$ of $\mathrm{Ph}_{2} \mathrm{CNDipp}$.
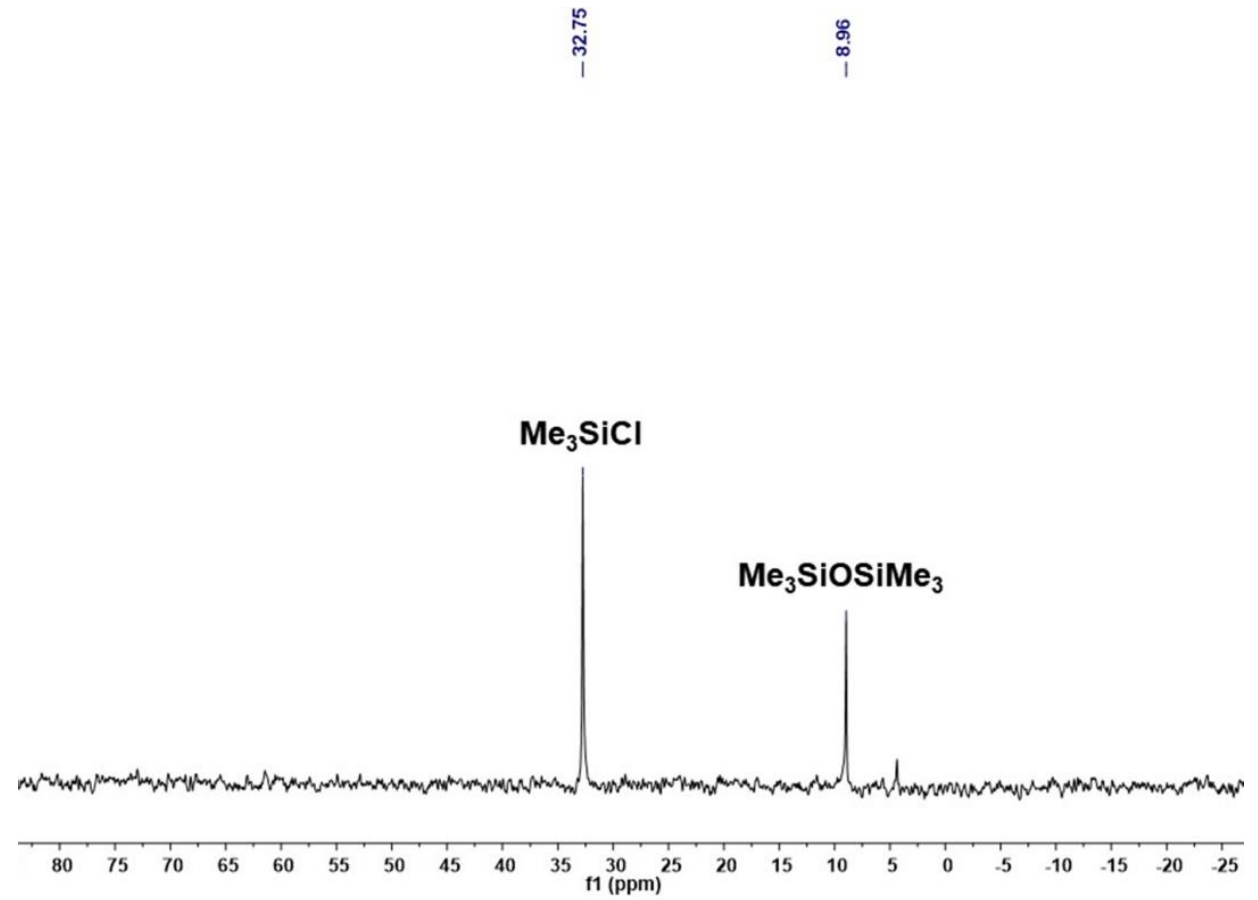

Figure S31. ${ }^{29} \mathrm{Si} \mathrm{NMR}$ spectrum $\left(99 \mathrm{MHz}, 25{ }^{\circ} \mathrm{C}\right.$, THF- $\left.d_{8}\right)$ of the reaction of the proposed ironoxo species with excess $\mathrm{Me}_{3} \mathrm{SiCl}$. 


\section{Kinetic plots}

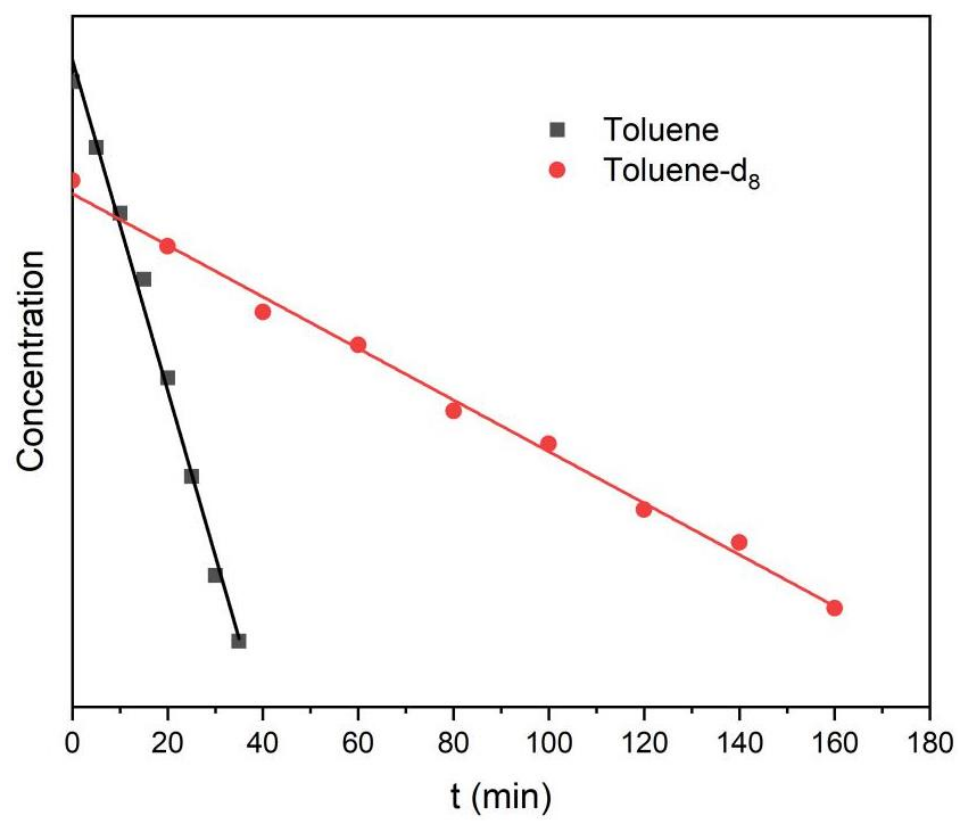

Figure S32. Plot of the concentration changes of $\left[\mathrm{Ph}_{2} \mathrm{~B}\left({ }^{t} \mathrm{BuIm}\right)_{2} \mathrm{FeNDipp}\right]\left[\mathrm{K}(18-\mathrm{C}-6) \mathrm{THF}_{2}\right](\mathbf{5})$ in the reaction with toluene/toluene- $d_{8}$ versus reaction time.The measured KIE is 6.4.

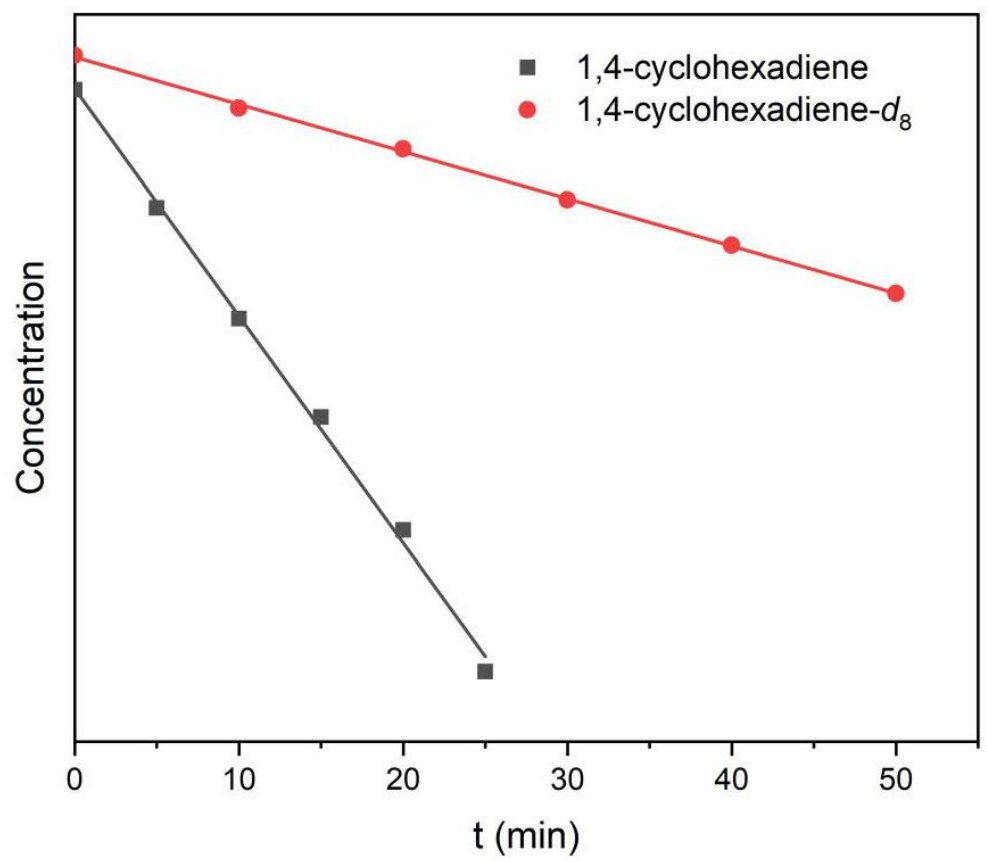

Figure S33. Plot of the concentration changes of $\left[\mathrm{Ph}{ }_{2} \mathrm{~B}\left({ }^{t} \mathrm{BuIm}\right)_{2} \mathrm{FeNDippK}\right]_{2}(4)$ in the reaction with 1,4-cyclohexadiene/1,4-cyclohexadiene- $d_{8}$ versus reaction time. The measured KIE is 4.8 . 


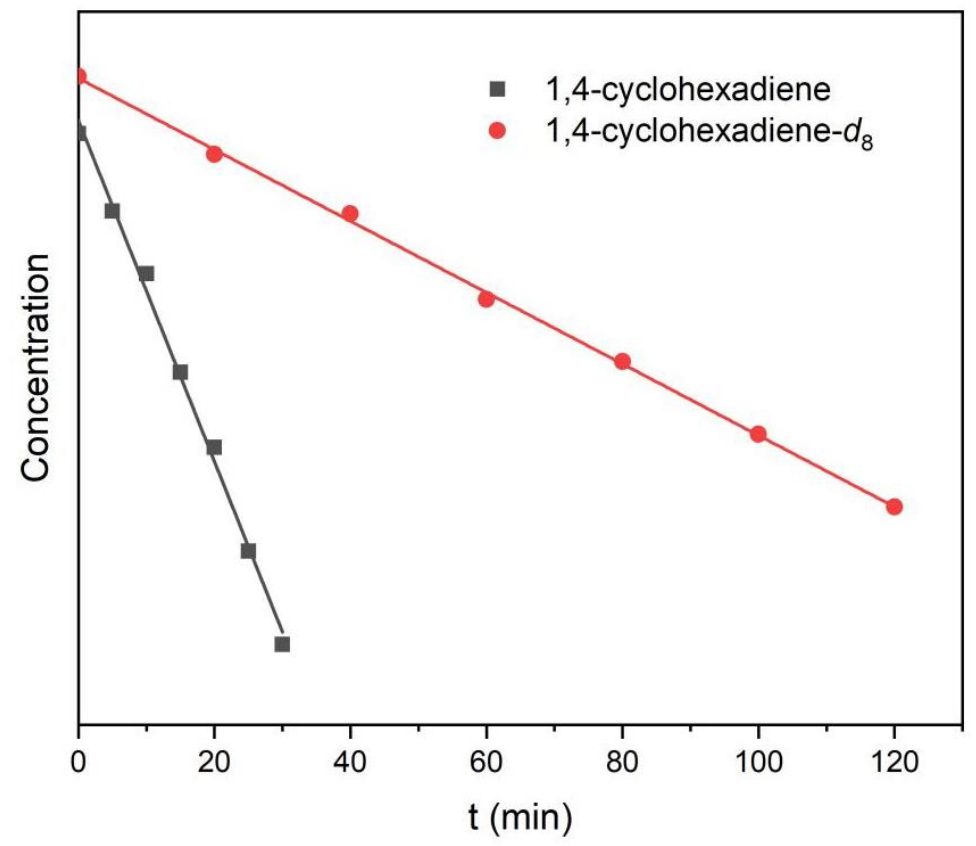

Figure S34. Plot of the concentration changes of $\left[\mathrm{Ph}{ }_{2} \mathrm{~B}\left({ }^{t} \mathrm{BuIm}\right)_{2} \mathrm{FeNDippNa}(\mathrm{THF})_{3}\right](3)$ in the reaction with 1,4-cyclohexadiene/1,4-cyclohexadiene- $d_{8}$ versus reaction time.The measured KIE is 4.8 . 


\section{Mössbauer spectra}

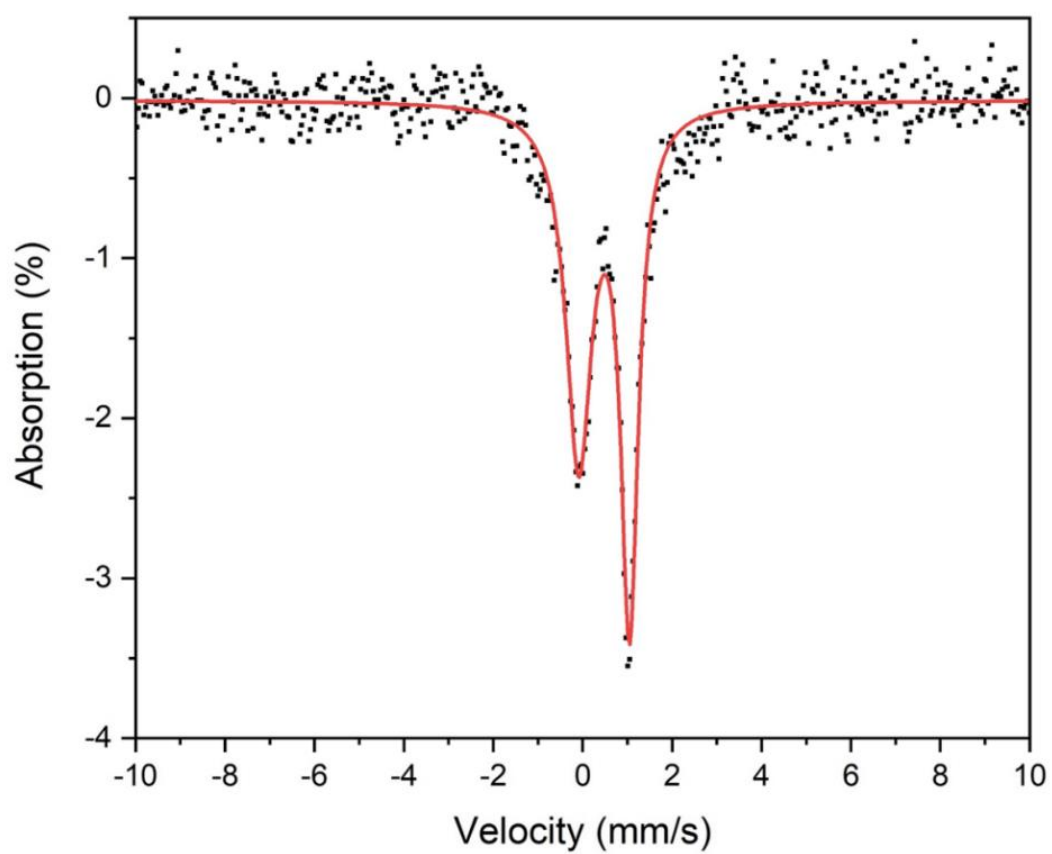

Figure S35. Zero-field ${ }^{57} \mathrm{Fe}$ Mössbauer spectrum of $\left[\mathrm{Ph}{ }_{2} \mathrm{~B}\left({ }^{t} \mathrm{BuIm}\right)_{2} \mathrm{FeNDippLi}(\mathrm{THF})_{2}\right]$ (2) measured at $80 \mathrm{~K}$. Parameters: $\delta=0.48 \mathrm{~mm} / \mathrm{s},\left|\Delta E_{\mathrm{Q}}\right|=1.14 \mathrm{~mm} / \mathrm{s}$.

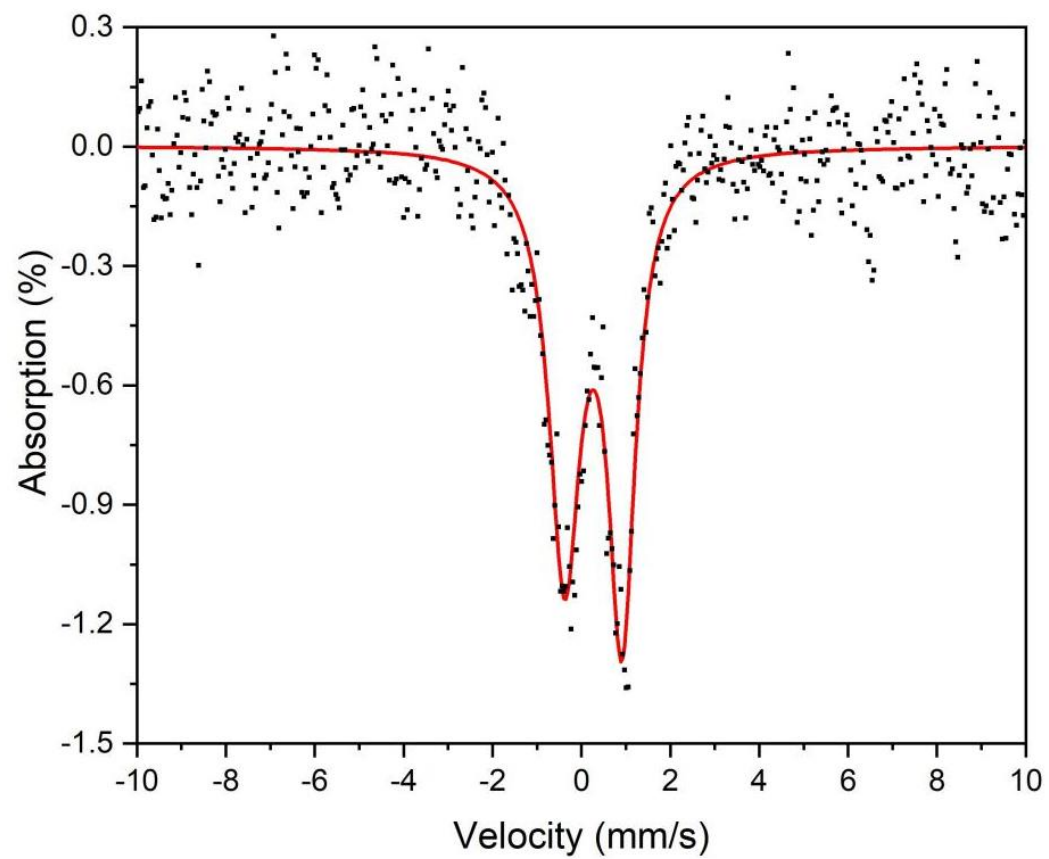

Figure S36. Zero-field ${ }^{57} \mathrm{Fe}$ Mössbauer spectrum of $\left[\mathrm{Ph}{ }_{2} \mathrm{~B}\left({ }^{t} \mathrm{BuIm}\right)_{2} \mathrm{FeNDippLi}(\mathrm{THF})_{2}\right]$ (2) measured at $220 \mathrm{~K}$. Parameters: $\delta=0.30 \mathrm{~mm} / \mathrm{s},\left|\Delta E_{\mathrm{Q}}\right|=1.28 \mathrm{~mm} / \mathrm{s}$. 


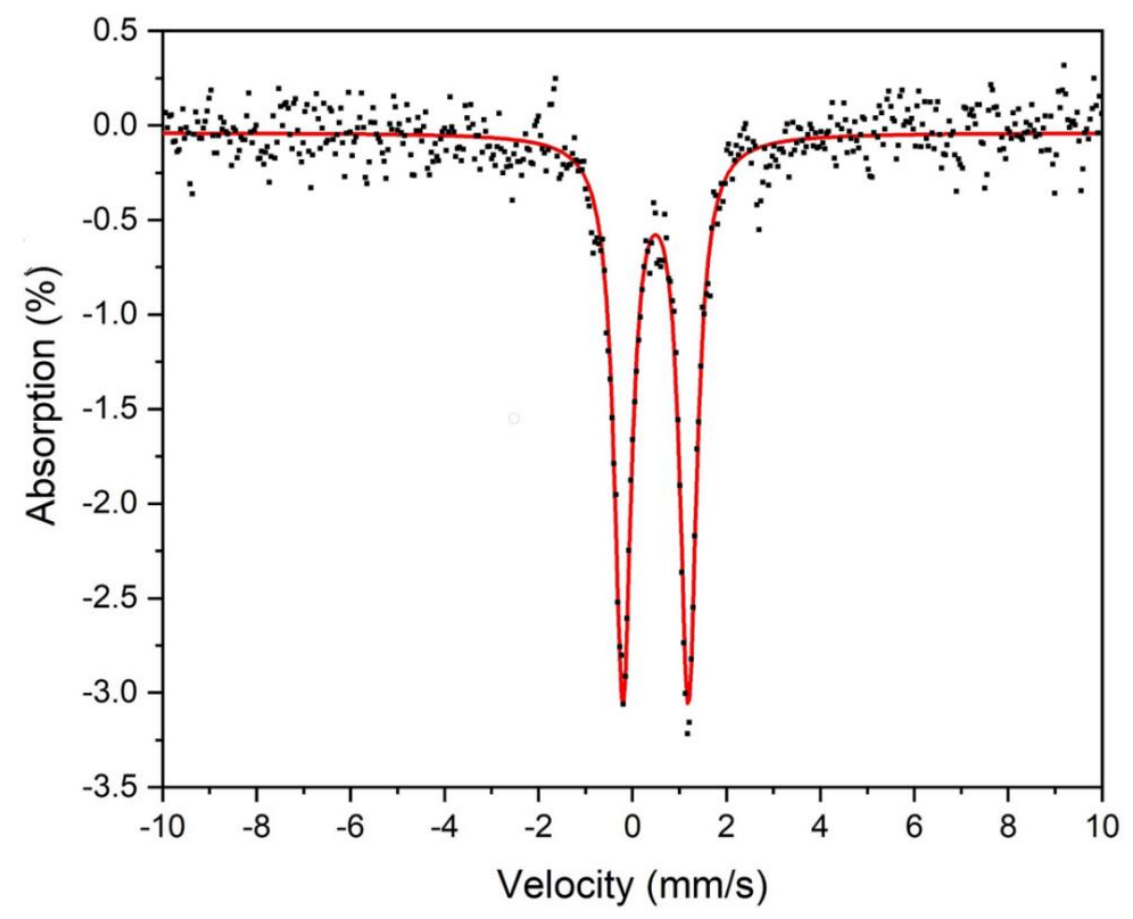

Figure S37. Zero-field ${ }^{57} \mathrm{Fe}$ Mössbauer spectrum of $\left[\mathrm{Ph}_{2} \mathrm{~B}\left({ }^{t} \mathrm{BuIm}\right)_{2} \mathrm{FeNDippNa}(\mathrm{THF})_{3}\right]$ (3) measured at $80 \mathrm{~K}$. Parameters: $\delta=0.49 \mathrm{~mm} / \mathrm{s},\left|\Delta E_{\mathrm{Q}}\right|=1.39 \mathrm{~mm} / \mathrm{s}$.

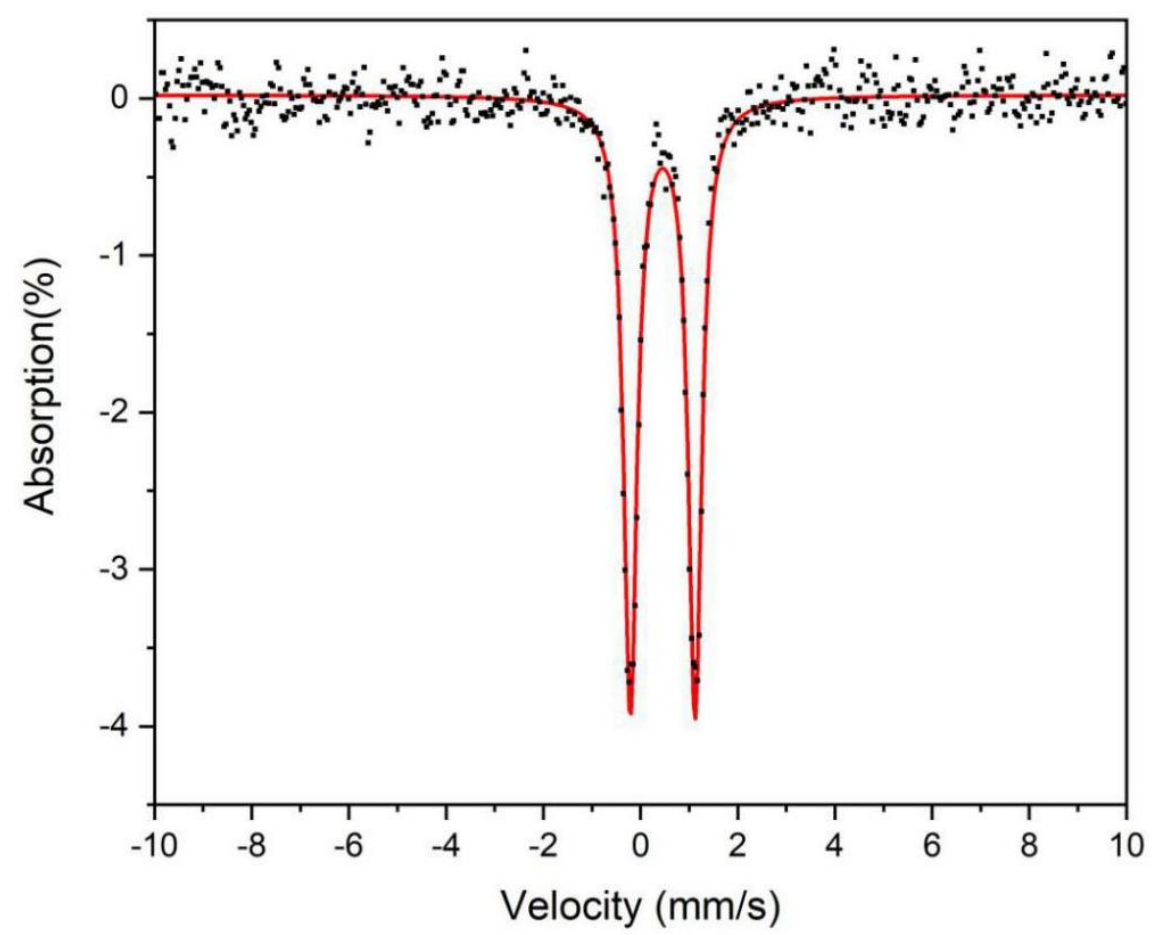

Figure S38. Zero-field ${ }^{57} \mathrm{Fe}$ Mössbauer spectrum of $\left[\mathrm{Ph}{ }_{2} \mathrm{~B}\left({ }^{t} \mathrm{BuIm}\right)_{2} \mathrm{FeNDippK}\right]_{2}(4)$ measured at $80 \mathrm{~K}$. Parameters: $\delta=0.46 \mathrm{~mm} / \mathrm{s},\left|\Delta E_{\mathrm{Q}}\right|=1.33 \mathrm{~mm} / \mathrm{s}$. 


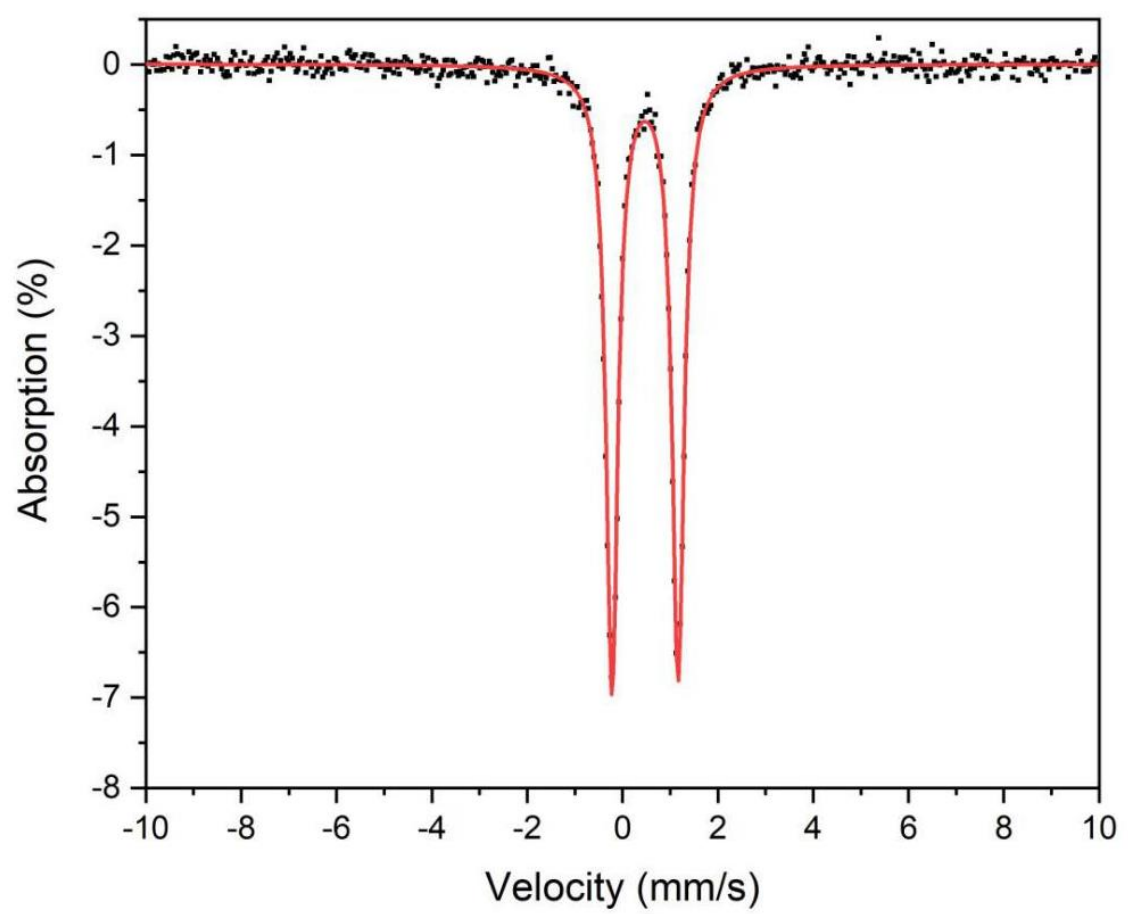

Figure S39. Zero-field ${ }^{57} \mathrm{Fe}$ Mössbauer spectrum of $\left[\mathrm{Ph}_{2} \mathrm{~B}\left({ }^{t} \mathrm{BuIm}\right)_{2} \mathrm{FeNHDipp}\right](\mathbf{6})$ measured at 80 K. Parameters: $\delta=0.47 \mathrm{~mm} / \mathrm{s},\left|\Delta E_{\mathrm{Q}}\right|=1.39 \mathrm{~mm} / \mathrm{s}$.

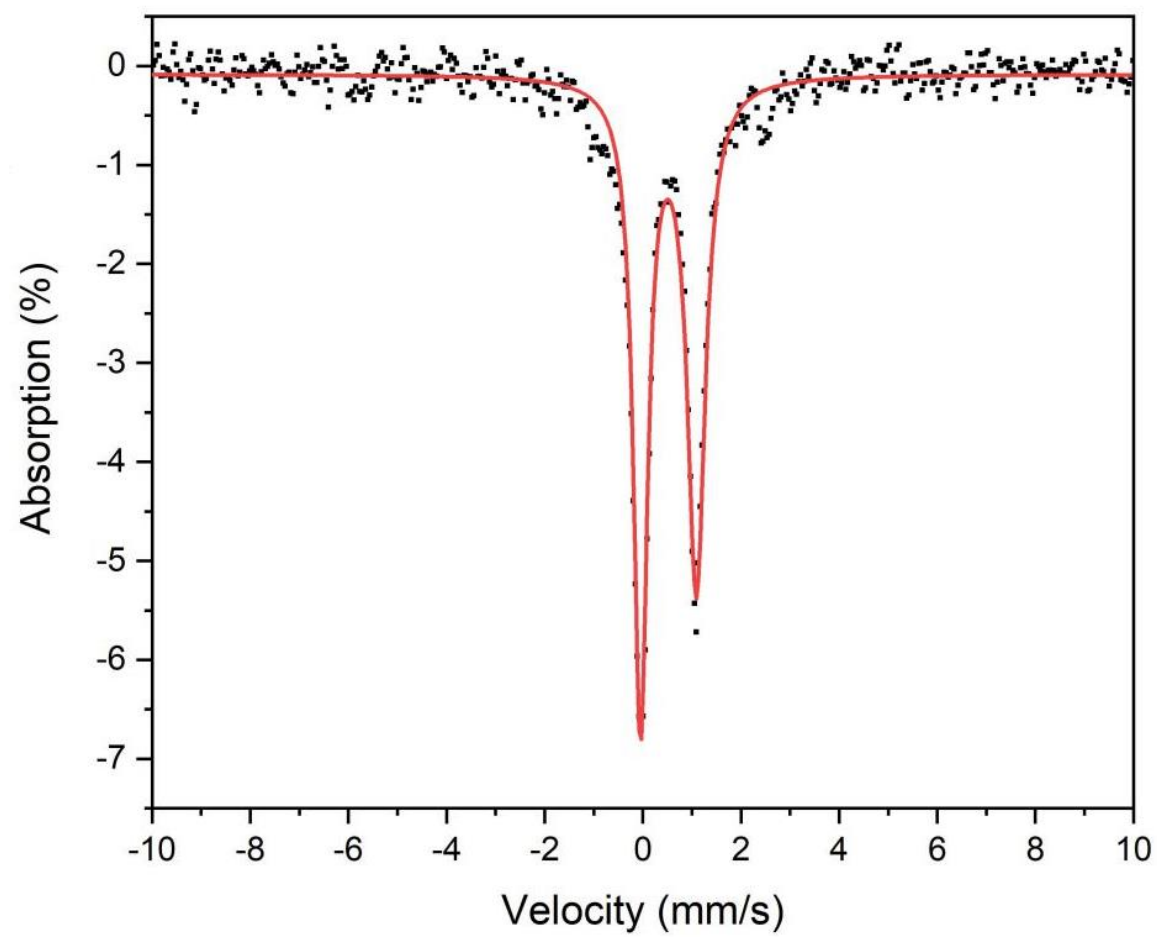

Figure S40. Zero-field ${ }^{57} \mathrm{Fe}$ Mössbauer spectrum of $\left[\mathrm{Ph}_{2} \mathrm{~B}\left({ }^{t} \mathrm{BuIm}\right)_{2} \mathrm{FeN}\left(\mathrm{CH}_{2} \mathrm{Ph}\right) \mathrm{Dipp}\right]$ (7) measured at $80 \mathrm{~K}$. Parameters: $\delta=0.52 \mathrm{~mm} / \mathrm{s},\left|\Delta E_{\mathrm{Q}}\right|=1.14 \mathrm{~mm} / \mathrm{s}$. 


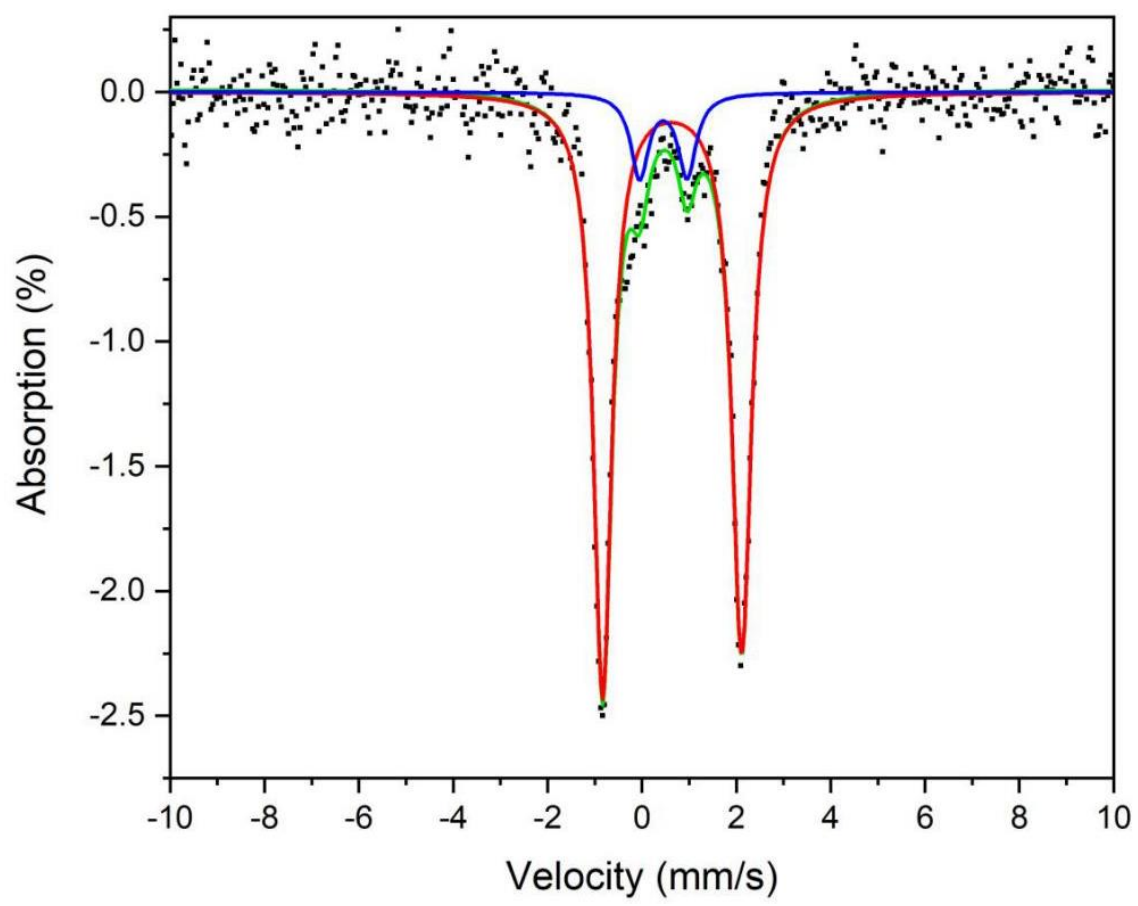

Figure S41. Zero-field ${ }^{57} \mathrm{Fe}$ Mössbauer spectrum of $\left[\mathrm{Ph}_{2} \mathrm{~B}\left({ }^{t} \mathrm{BuIm}\right)_{2} \mathrm{FeNHDipp}\right]\left[\mathrm{K}(18-\mathrm{C}-6) \mathrm{THF}_{2}\right]$ (9-[K(18-C-6)]) measured at $80 \mathrm{~K}$. Parameters: $\delta=0.64 \mathrm{~mm} / \mathrm{s},\left|\Delta E_{\mathrm{Q}}\right|=2.95 \mathrm{~mm} / \mathrm{s}$. The minor component (blue line) comes from the unreacted $\left[\mathrm{Ph} 2 \mathrm{~B}\left({ }^{t} \mathrm{BuIm}\right)_{2} \mathrm{FeNDipp}\right]\left[\mathrm{K}(18-\mathrm{C}-6) \mathrm{THF}_{2}\right](\mathbf{5})$.

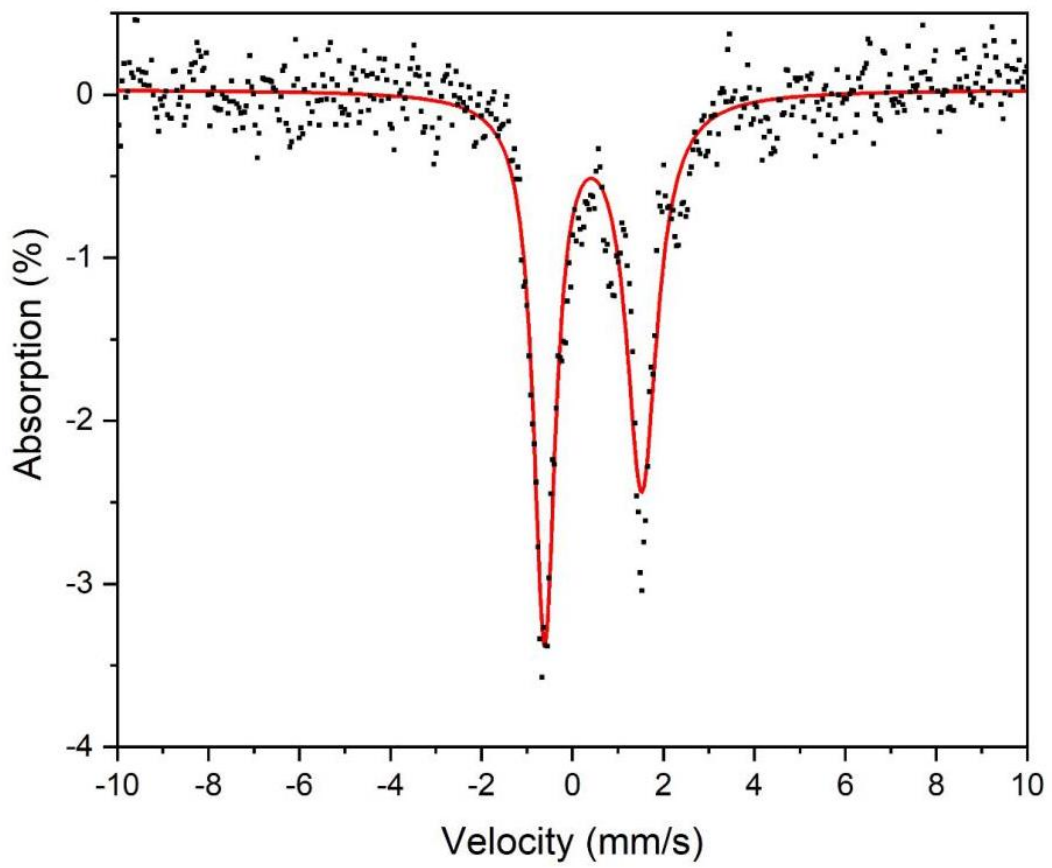

Figure S42. Zero-field ${ }^{57} \mathrm{Fe}$ Mössbauer spectrum of $\left[\mathrm{Ph}_{2} \mathrm{~B}\left({ }^{t} \mathrm{BuIm}\right)_{2} \mathrm{Fe}\left(\mathrm{N}(\mathrm{Dipp}) \mathrm{C}(\mathrm{Ph})_{2} \mathrm{O}\right)\right][\mathrm{K}(18-$ C-6)] (10) measured at $80 \mathrm{~K}$. Parameters: $\delta=0.46 \mathrm{~mm} / \mathrm{s},\left|\Delta E_{\mathrm{Q}}\right|=2.13 \mathrm{~mm} / \mathrm{s}$. 


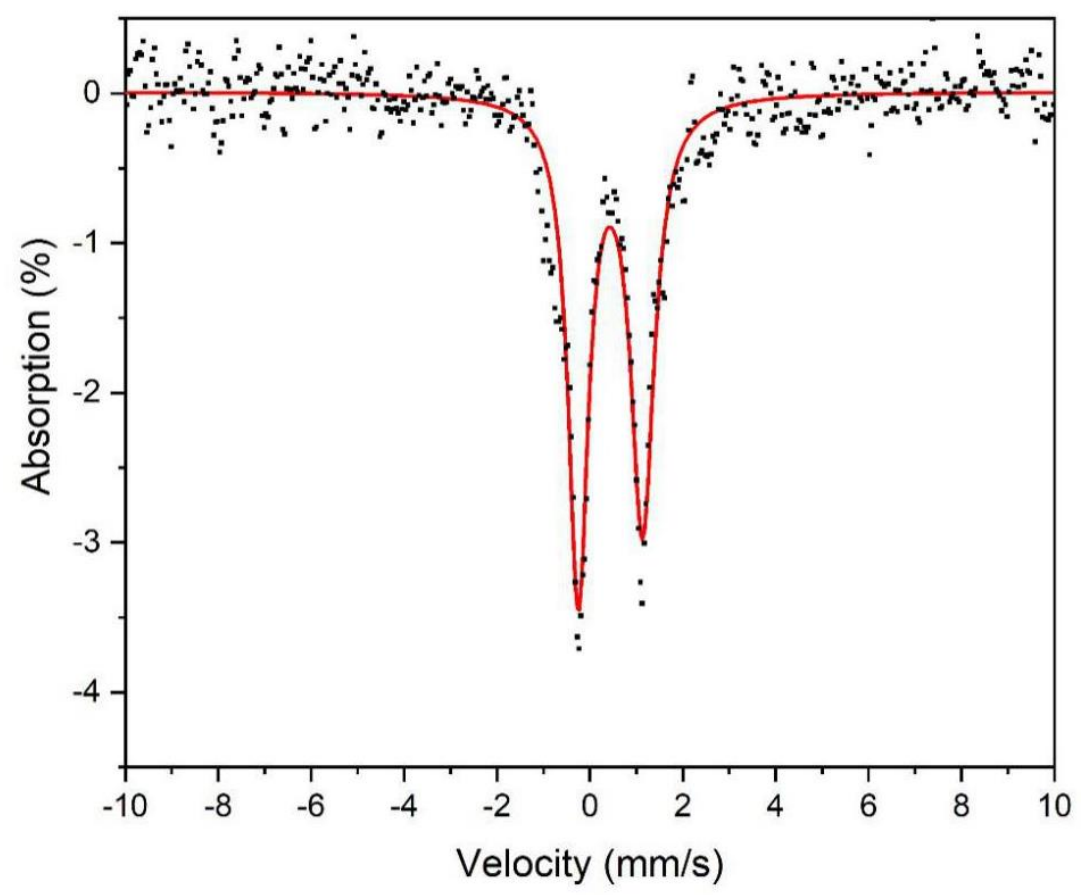

Figure S43. Zero-field ${ }^{57} \mathrm{Fe}$ Mössbauer spectrum of $\left[\mathrm{Ph}_{2} \mathrm{~B}\left({ }^{t} \mathrm{BuIm}\right)_{2} \mathrm{FeN}(\mathrm{Dipp}) \mathrm{C}(\mathrm{Ph})_{2} \mathrm{OK}(\mathrm{THF})_{\mathrm{x}}\right]$ (11) measured at $80 \mathrm{~K}$. Parameters: $\delta=0.44 \mathrm{~mm} / \mathrm{s},\left|\Delta E_{\mathrm{Q}}\right|=1.38 \mathrm{~mm} / \mathrm{s}$.

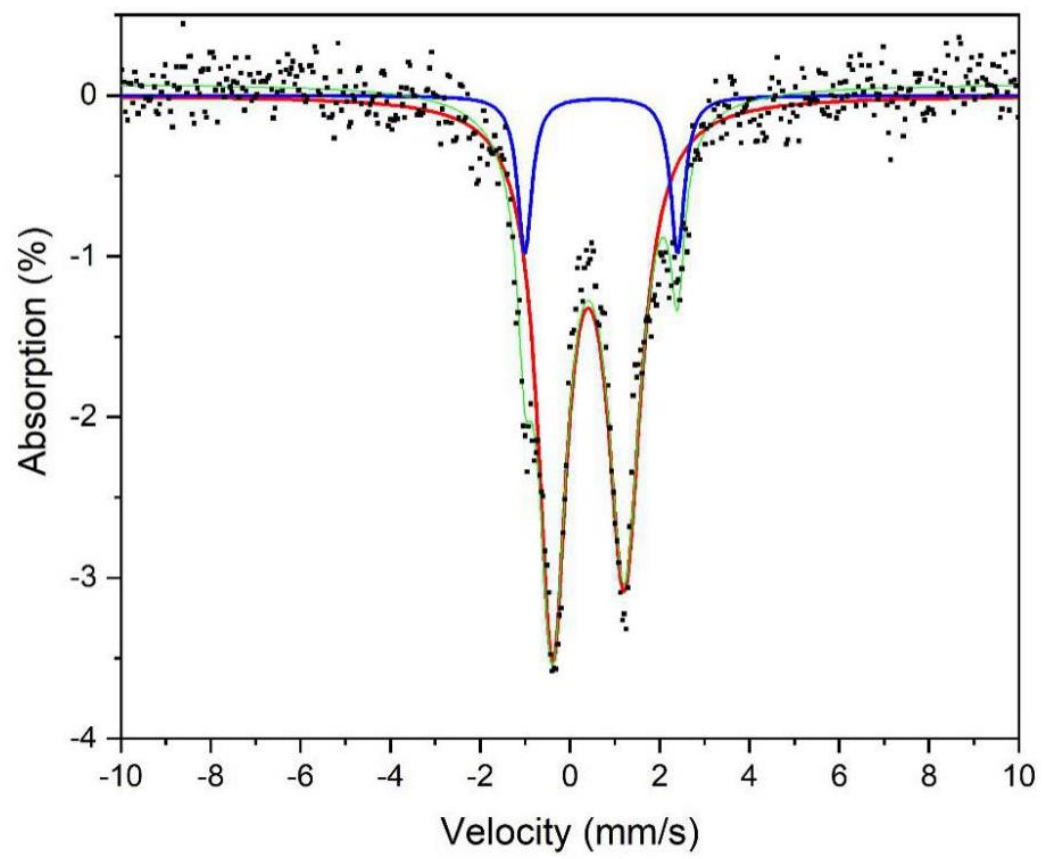

Figure S44. Zero-field ${ }^{57} \mathrm{Fe}$ Mössbauer spectrum of $\left[\mathrm{Ph} 2 \mathrm{~B}\left({ }^{t} \mathrm{BuIm}\right)_{2} \mathrm{FeN}(\mathrm{Dipp}) \mathrm{C}(\mathrm{Ph})_{2} \mathrm{ONa}(\mathrm{THF})_{\mathrm{x}}\right]$ (12) measured at $80 \mathrm{~K}$. Parameters: $\delta=0.41 \mathrm{~mm} / \mathrm{s},\left|\Delta E_{\mathrm{Q}}\right|=1.60 \mathrm{~mm} / \mathrm{s}$. The minor component (blue line $10 \%$ ) is an unidentified iron complex with $\delta=0.70 \mathrm{~mm} / \mathrm{s},\left|\Delta E_{\mathrm{Q}}\right|=3.40 \mathrm{~mm} / \mathrm{s}$. 


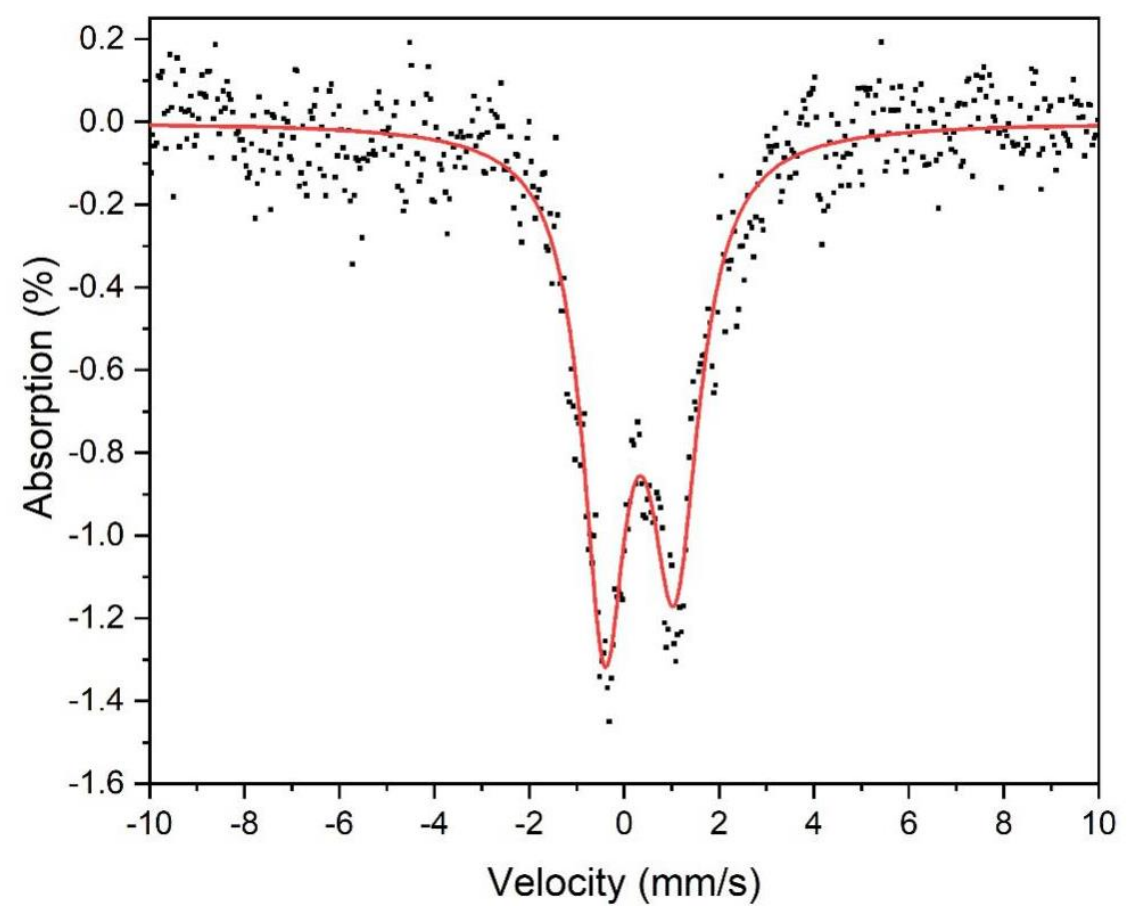

Figure S45. Zero-field ${ }^{57} \mathrm{Fe}$ Mössbauer spectrum of $\left[\mathrm{Ph}{ }_{2} \mathrm{~B}\left({ }^{t} \mathrm{BuIm}\right)_{2} \mathrm{FeN}(\mathrm{Dipp}) \mathrm{C}(\mathrm{Ph})_{2} \mathrm{OLi}(\mathrm{THF})_{\mathrm{x}}\right]$ (13) measured at $80 \mathrm{~K}$. Parameters: $\delta=0.32 \mathrm{~mm} / \mathrm{s},\left|\Delta E_{\mathrm{Q}}\right|=1.48 \mathrm{~mm} / \mathrm{s}$.

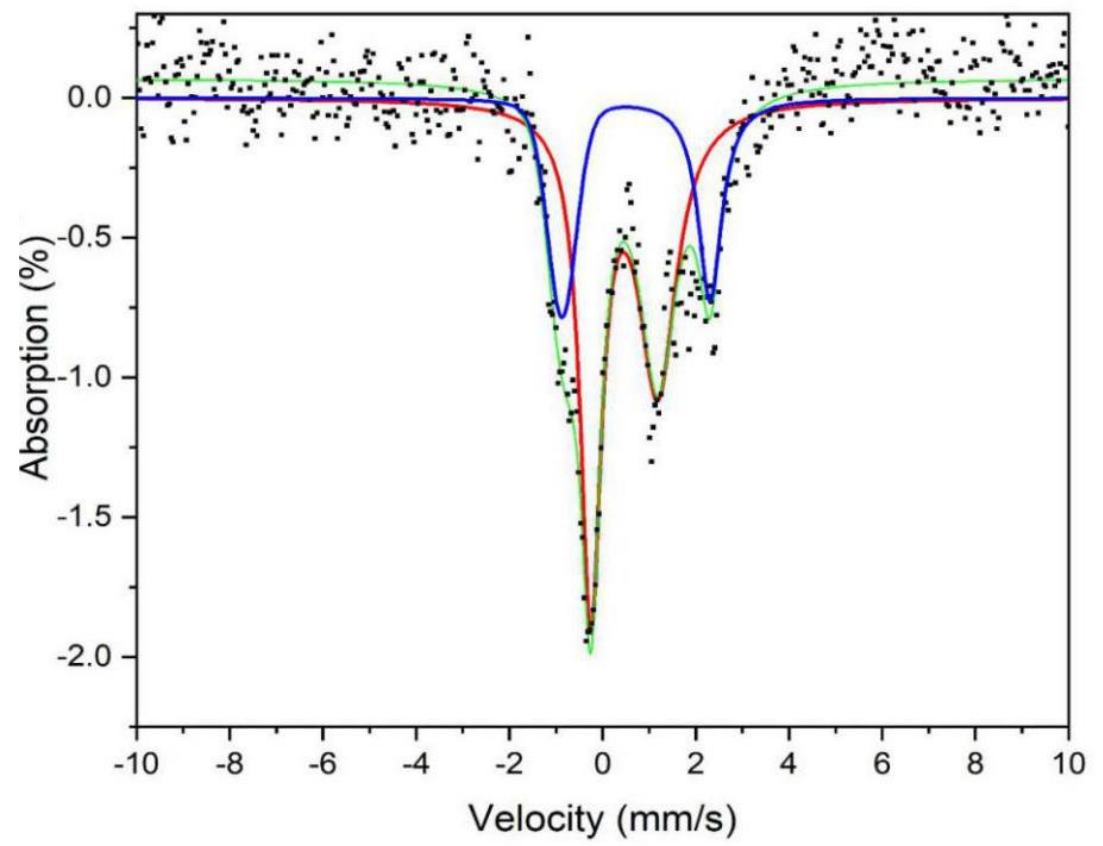

Figure S46. Zero-field ${ }^{57} \mathrm{Fe}$ Mössbauer spectrum of the proposed iron oxo species from the decomposition of $\left[\mathrm{Ph}_{2} \mathrm{~B}\left({ }^{t} \mathrm{BuIm}\right)_{2} \mathrm{FeN}(\mathrm{Dipp}) \mathrm{C}(\mathrm{Ph})_{2} \mathrm{OK}(\mathrm{THF})_{\mathrm{x}}\right](\mathbf{1 1})$ measured at $80 \mathrm{~K}$. The major component (red line $70 \%$ ), parameters: $\delta=0.47 \mathrm{~mm} / \mathrm{s},\left|\Delta E_{\mathrm{Q}}\right|=1.43 \mathrm{~mm} / \mathrm{s}$. The minor component (blue line 30\%), parameters: $\delta=0.72 \mathrm{~mm} / \mathrm{s},\left|\Delta E_{\mathrm{Q}}\right|=3.17 \mathrm{~mm} / \mathrm{s}$. 


\section{UV-vis spectra}

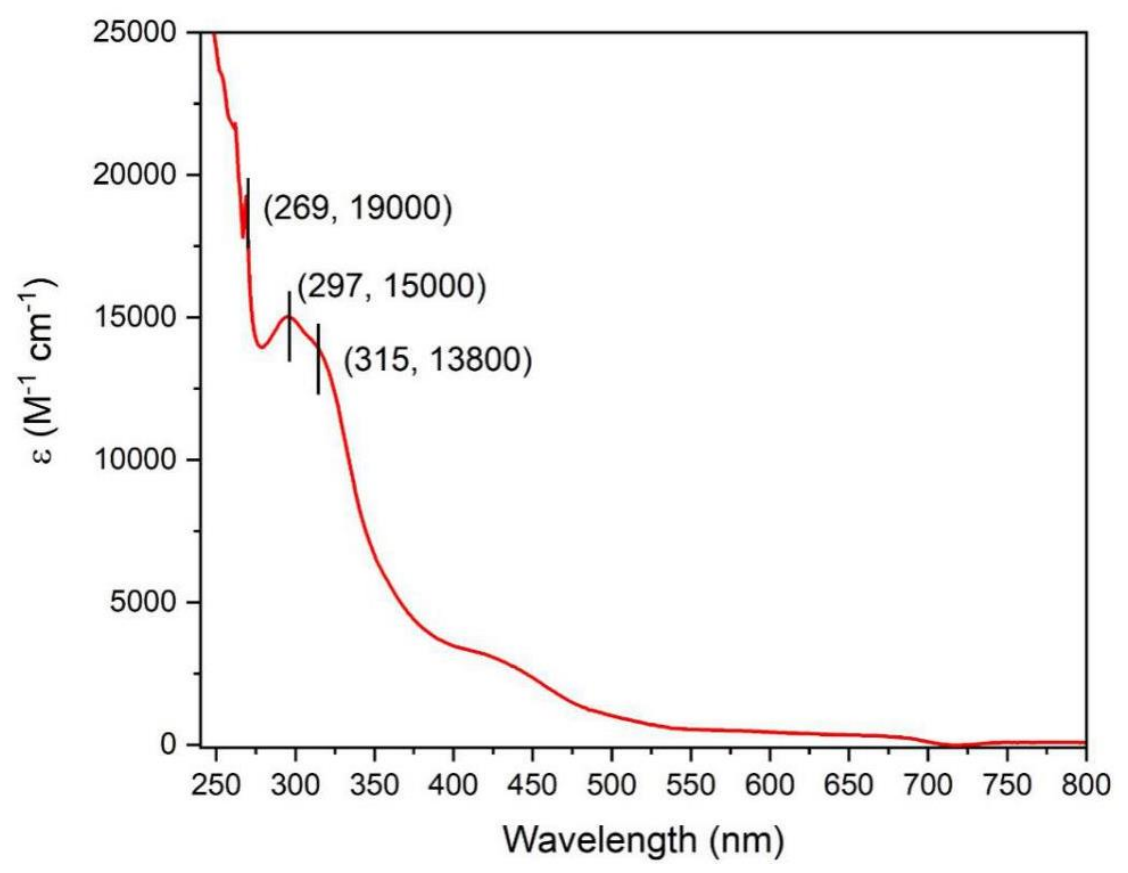

Figure S47. UV-vis spectrum of $\left[\mathrm{Ph}_{2} \mathrm{~B}\left({ }^{t} \mathrm{BuIm}\right)_{2} \mathrm{FeNDippLi}(\mathrm{THF})_{2}\right](2)$ measured at $25^{\circ} \mathrm{C}$ in $\mathrm{THF}$.

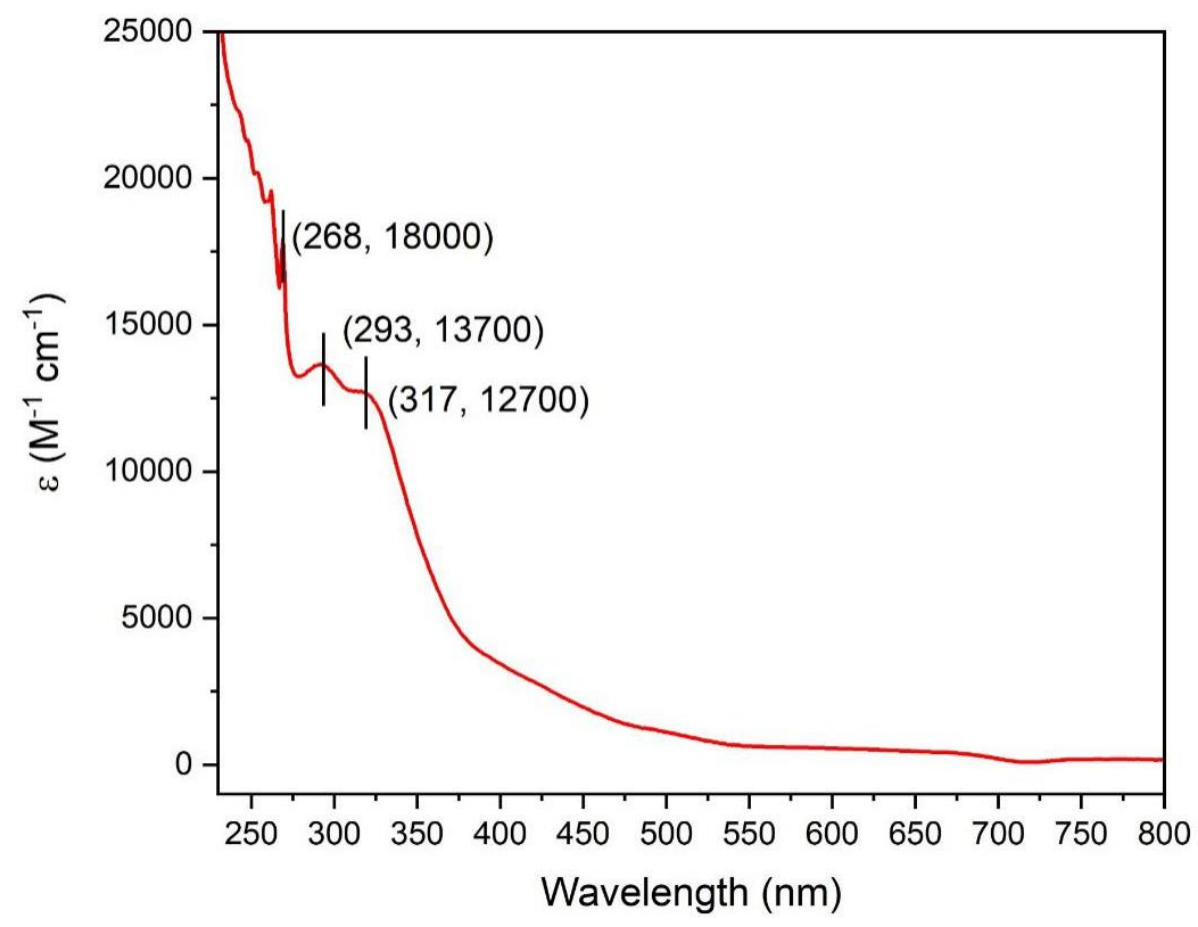

Figure S48. UV-vis spectrum of $\left[\mathrm{Ph}_{2} \mathrm{~B}\left({ }^{t} \mathrm{BuIm}\right)_{2} \mathrm{FeNDippNa}(\mathrm{THF})_{3}\right](\mathbf{3})$ measured at $25^{\circ} \mathrm{C}$ in $\mathrm{THF}$. 


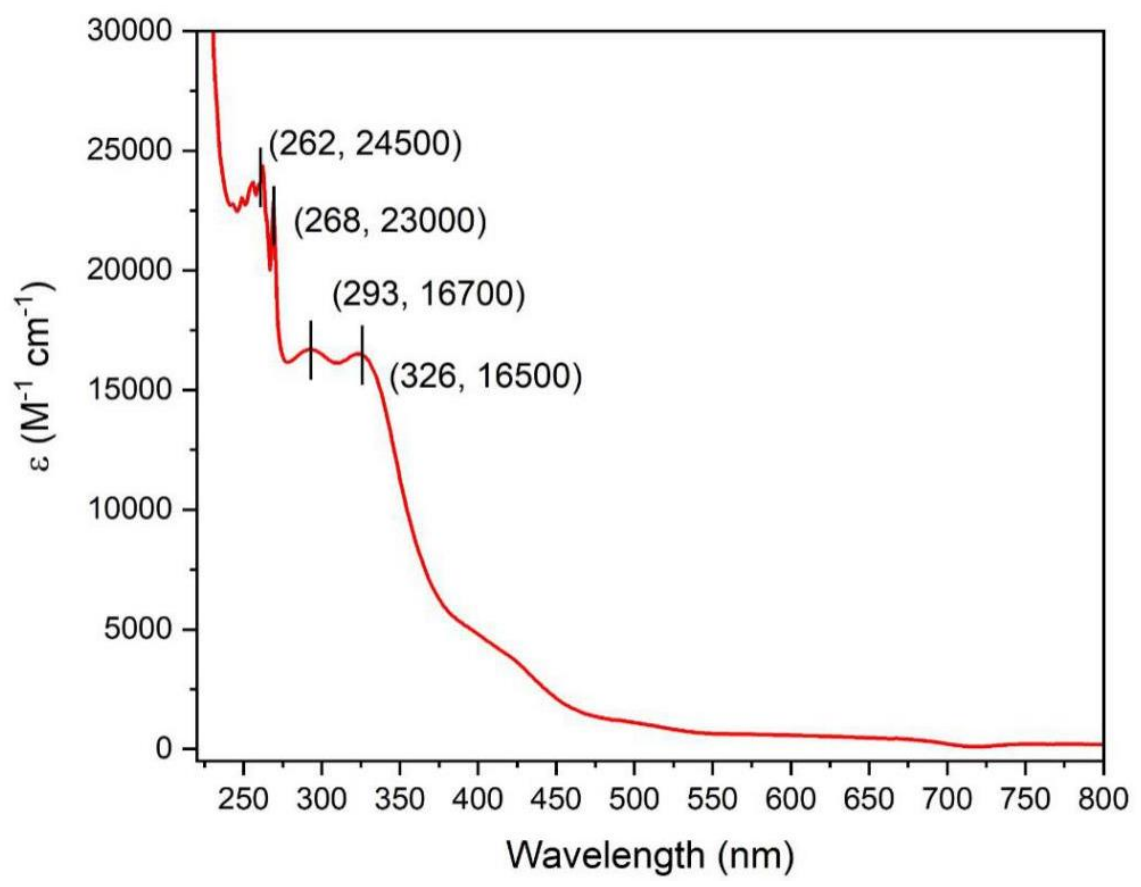

Figure S49. UV-vis spectrum of $\left[\mathrm{Ph}_{2} \mathrm{~B}\left({ }^{t} \mathrm{BuIm}\right)_{2} \mathrm{FeNDippK}\right]_{2}(4)$ measured at $25^{\circ} \mathrm{C}$ in $\mathrm{THF}$.

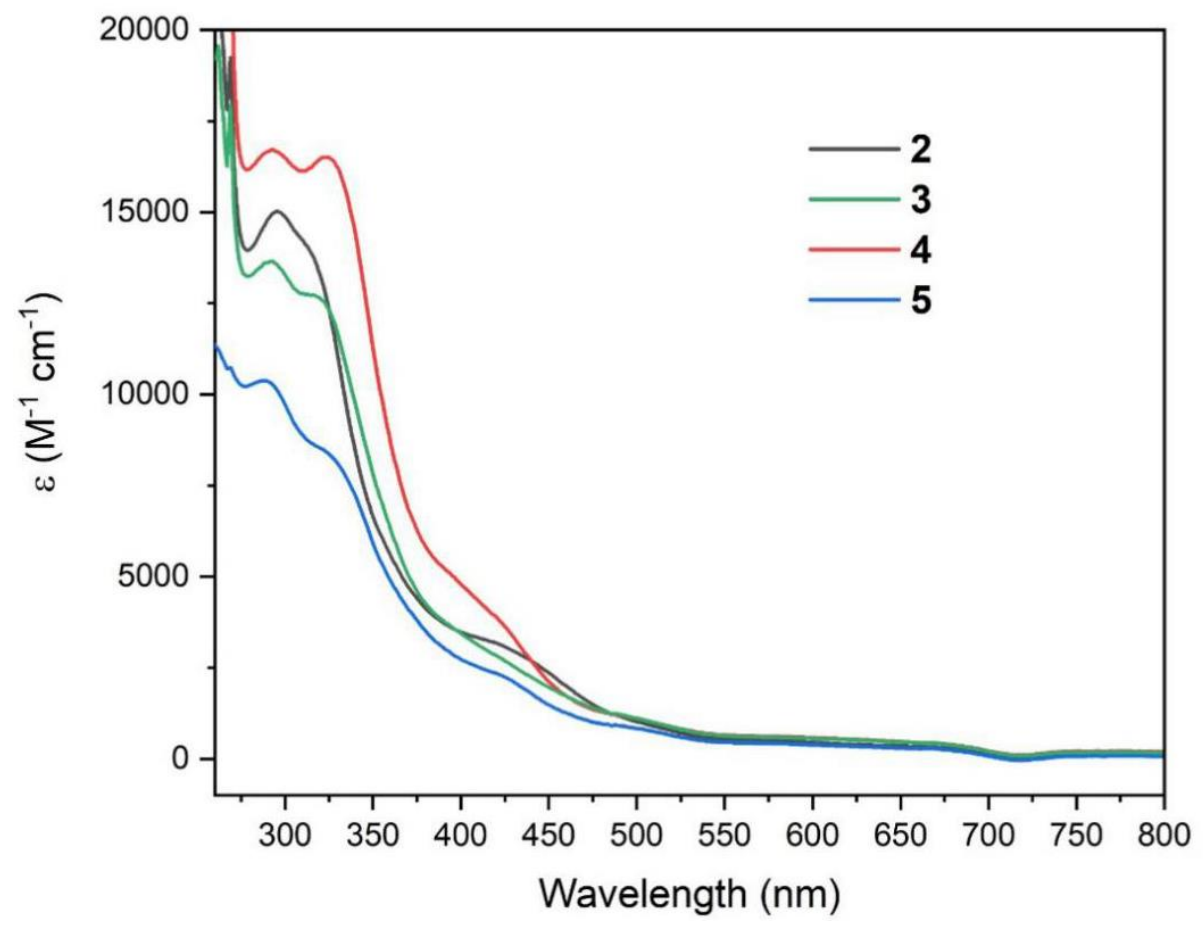

Figure S50. The comparision of UV-vis spectrum of $\left[\mathrm{Ph}_{2} \mathrm{~B}\left({ }^{t} \mathrm{BuIm}\right)_{2} \mathrm{FeNDippLi}(\mathrm{THF})_{2}\right]$ (2), $\left[\mathrm{Ph}_{2} \mathrm{~B}\left({ }^{t} \mathrm{BuIm}\right)_{2} \mathrm{FeNDippNa}(\mathrm{THF})_{3}\right]$ (3), $\left[\mathrm{Ph}{ }_{2} \mathrm{~B}\left({ }^{t} \mathrm{BuIm}\right)_{2} \mathrm{FeNDippK}\right]_{2}$

(4) and $\left[\mathrm{Ph}_{2} \mathrm{~B}\left({ }^{t} \mathrm{BuIm}\right)_{2} \mathrm{FeNDipp}\right]\left[\mathrm{K}(18-\mathrm{C}-6) \mathrm{THF}_{2}\right](5)$ measured at $25^{\circ} \mathrm{C}$ in THF. 


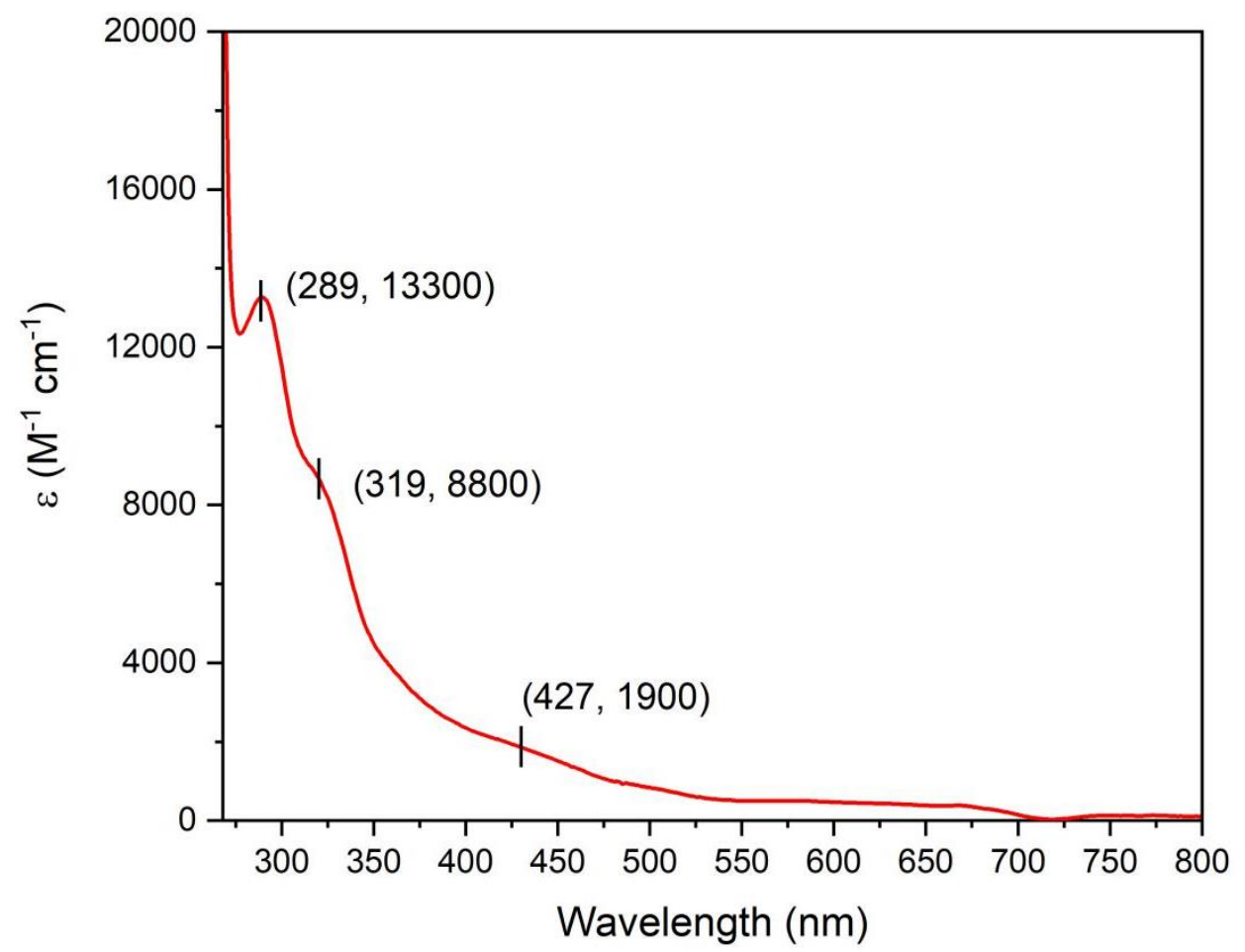

Figure S51. UV-vis spectrum of $\left[\mathrm{Ph}_{2} \mathrm{~B}\left({ }^{t} \mathrm{BuIm}\right)_{2} \mathrm{FeNHDipp}\right](6)$ measured at $25{ }^{\circ} \mathrm{C}$ in $\mathrm{THF}$.

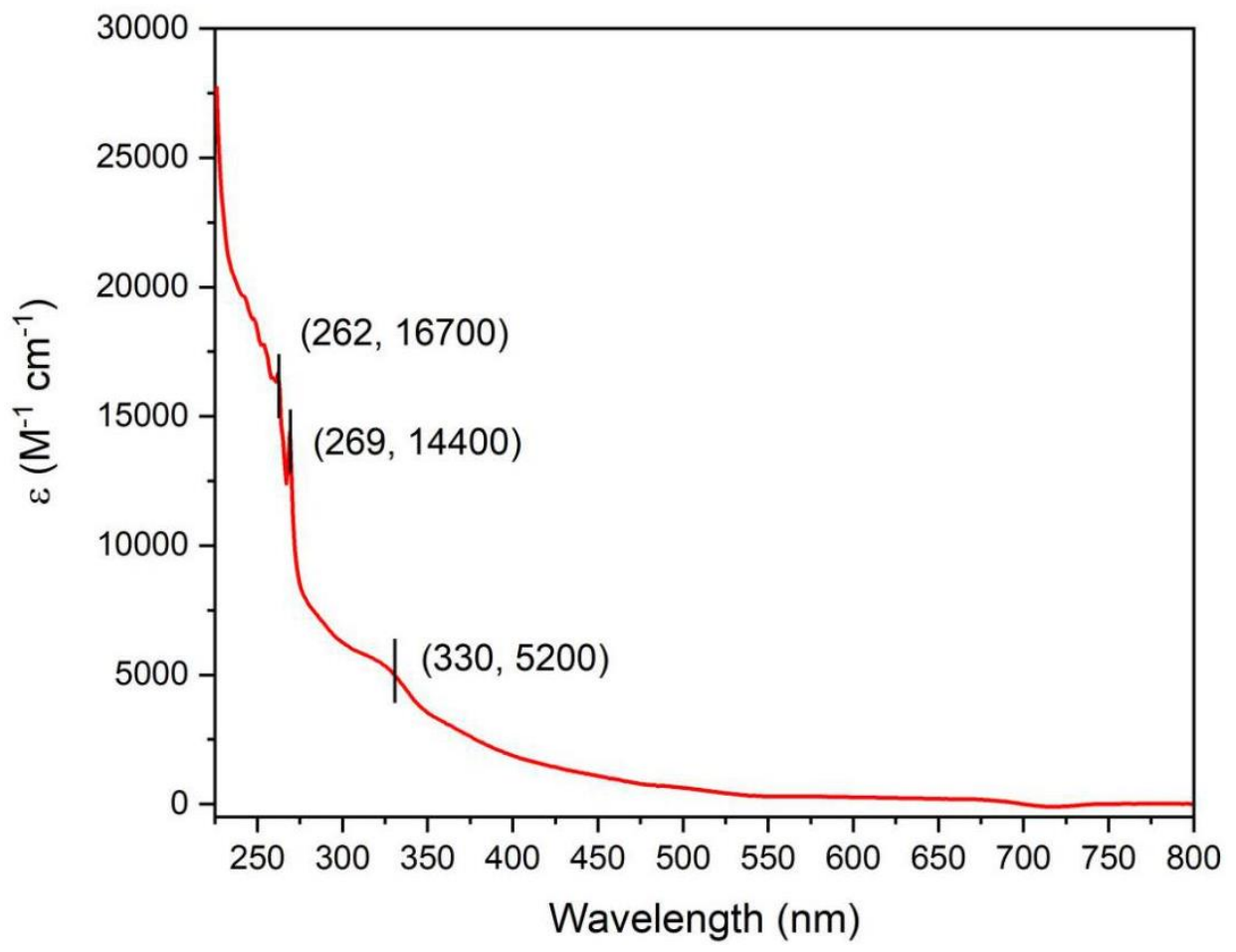

Figure S52. UV-vis spectrum of $\left[\mathrm{Ph}_{2} \mathrm{~B}\left({ }^{t} \mathrm{BuIm}\right)_{2} \mathrm{FeN}\left(\mathrm{CH}_{2} \mathrm{Ph}\right) \mathrm{Dipp}\right](7)$ measured at $25^{\circ} \mathrm{C}$ in $\mathrm{THF}$. 


\section{Cyclic voltammetry}

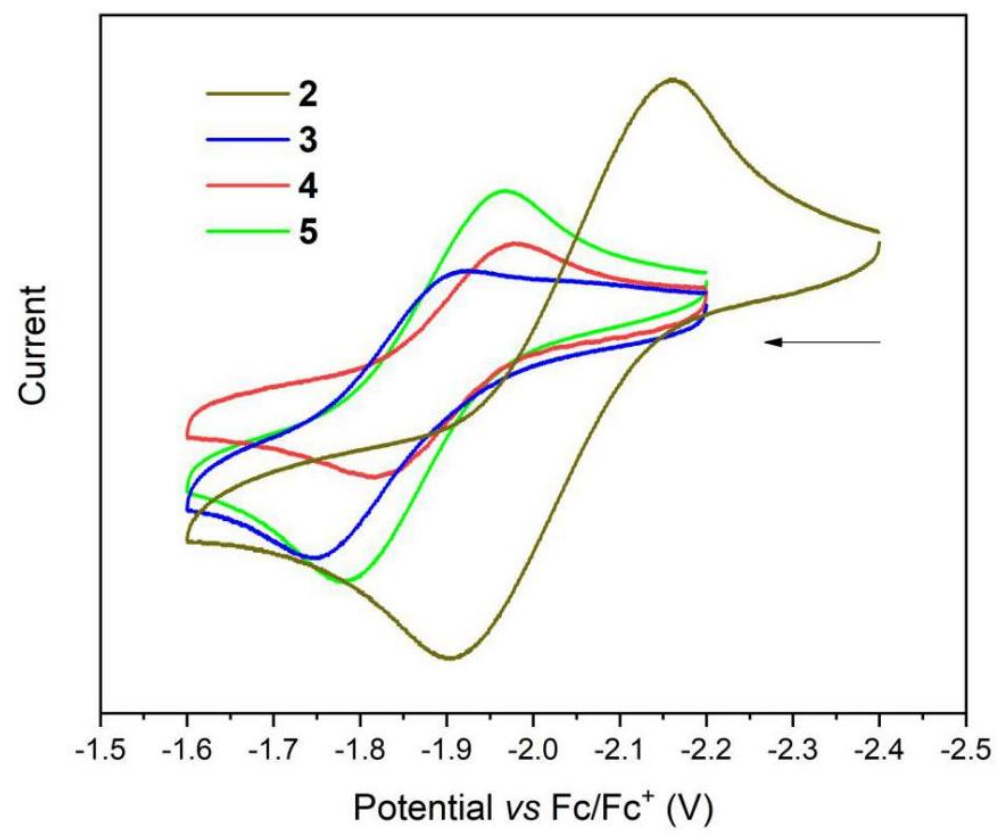

Figure S53. Cyclic voltammogram of the one electron oxidation of 2-5 measured in 0.1 M THF solution of $\left[{ }^{n} \mathrm{Bu}_{4} \mathrm{~N}\right]\left[\mathrm{PF}_{6}\right]$ under a $\mathrm{N}_{2}$ atmosphere with a scan rate of $200 \mathrm{mV} / \mathrm{s}$. The corresponding redox potentials are $\mathbf{2}, E_{1 / 2}=-2.03 \mathrm{~V} ; \mathbf{3}, E_{1 / 2}=-1.85 \mathrm{~V} ; \mathbf{4}, E_{1 / 2}=-1.90 \mathrm{~V} ; \mathbf{5}, E_{1 / 2}=-1.89 \mathrm{~V}$.

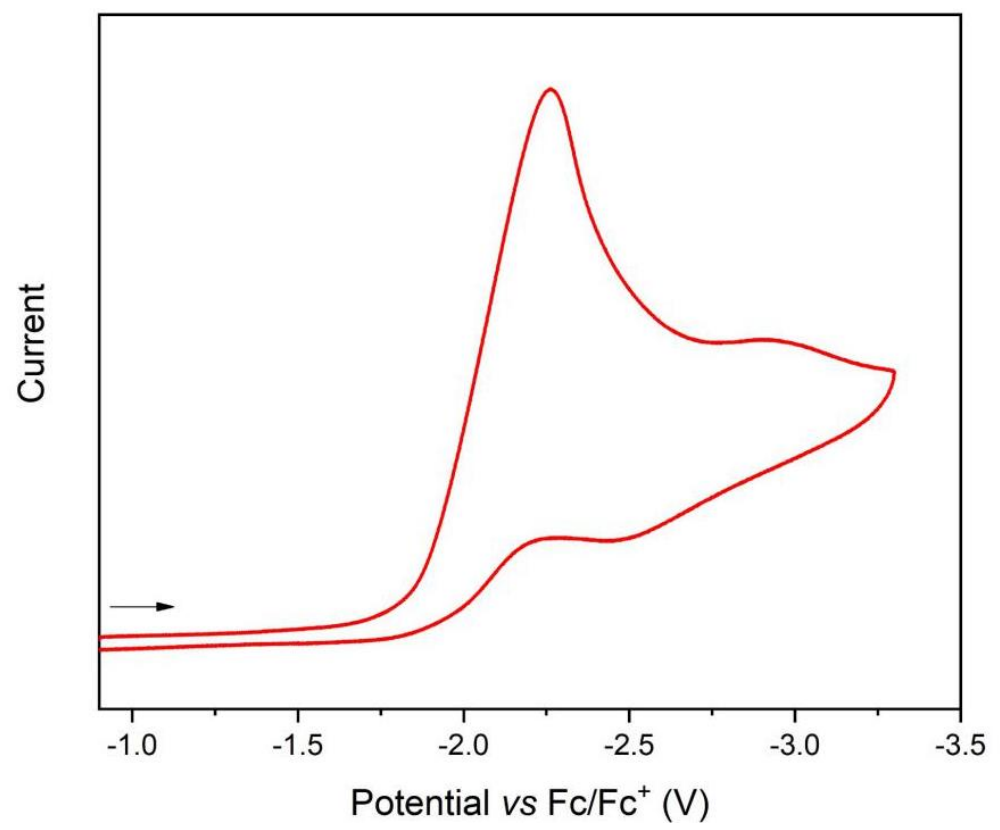

Figure S54. Cyclic voltammogram of $[\mathrm{LutH}][\mathrm{Cl}]$ measured in $0.1 \mathrm{M}$ THF solution of $\left[{ }^{n} \mathrm{Bu}_{4} \mathrm{~N}\right]\left[\mathrm{PF}_{6}\right]$ under a $\mathrm{N}_{2}$ atmosphere with a scan rate of $200 \mathrm{mV} / \mathrm{s}$. The peak potential $E_{\mathrm{p}, \mathrm{c}}=-2.26$ $\mathrm{V}$ and the half-peak potential $E_{\mathrm{p}, 1 / 2}=-2.00 \mathrm{~V}$. This gives $K_{\mathrm{eq}} \approx 0.01$ for the reaction with $\mathbf{5}$. 


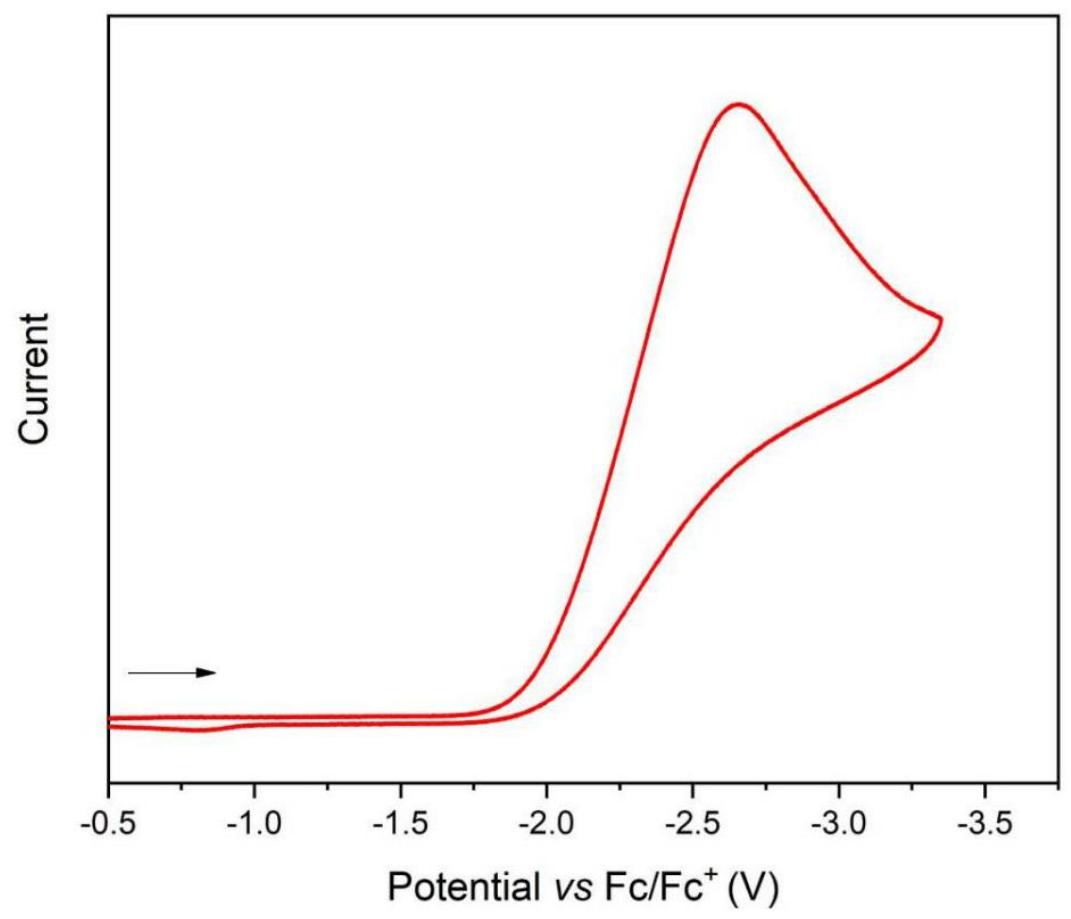

Figure S55. Cyclic voltammogram of $\mathrm{PhCH}_{2} \mathrm{Br}$ measured in $0.1 \mathrm{M}$ THF solution of $\left[{ }^{n} \mathrm{Bu}_{4} \mathrm{~N}\right]\left[\mathrm{PF}_{6}\right]$ under a $\mathrm{N}_{2}$ atmosphere with a scan rate of $200 \mathrm{mV} / \mathrm{s}$. The peak potential $E_{\mathrm{p}, \mathrm{c}}=-2.65 \mathrm{~V}$ and the half-peak potential $E_{\mathrm{p}, 1 / 2}=-2.27 \mathrm{~V}$. This gives $K_{\mathrm{eq}} \approx 3.7 \times 10^{-7}$ for the reaction with $\mathbf{5}$. 


\section{Computational Details}

All calculations were performed using density functional theory (DFT) as implemented in the Orca 4.2.1 computational software package. ${ }^{9}$ The structures of $\mathbf{2}, \mathbf{3}$ and model complexes $\mathbf{2}^{\prime}-\mathbf{4}^{\prime}(S=2)$ were optimized in the gas phase with the B3LYP functional and a hybrid def2-TZVP/SVP basis sets, including relativistic effects in zero order regular approximation (ZORA). ${ }^{10-11}$ Here, the Fe center and all atoms attached to Fe were treated with the def2-TZVP basis set for mathematical flexibility. Additionally, Grimme D3BJ dispersion corrections were included for all atoms. ${ }^{12-13}$ The structures of model complexes 2'-4' were verified to be minima on the potential energy surface by frequency calculations.

For N-H BDFE calculation, the structures of the model complexes $\mathrm{Me}_{2} \mathrm{~B}(\mathrm{MeIm}){ }_{2} \mathrm{Fe}-\mathrm{NHPh}(\mathrm{M})$ and $\mathrm{Me}_{2} \mathrm{~B}(\mathrm{MeIm})_{2} \mathrm{Fe}=\mathrm{NPh}(\mathrm{M})(\mathrm{M}=\mathrm{Li}, \mathrm{Na}, \mathrm{K})$ were optimized in the gas phase with the B3LYP functional, def2-SVP basis sets and Grimme D3BJ dispersion were included for all atoms. ${ }^{10-13}$ All structures were verified to be minima on the potential energy surface by frequency calculations. Reevaluation of the electronic energies (single point energy corrections) was done with the triple zeta basis set def2-TZVP. ${ }^{10-11}$ The gas phase $\mathrm{N}-\mathrm{H}$ BDFE for $\mathrm{Me}_{2} \mathrm{~B}(\mathrm{MeIm})_{2} \mathrm{Fe}-\mathrm{NHPh}(\mathrm{M})$ was determined using the ground state energy of the hydrogen atom ( -0.5 hartree) according to the following equation:

$$
\mathrm{Me}_{2} \mathrm{~B}(\mathrm{MeIm})_{2} \mathrm{Fe}=\mathrm{NPh}(\mathrm{M})+\mathrm{H}^{\bullet} \rightarrow \mathrm{Me}_{2} \mathrm{~B}(\mathrm{MeIm}){ }_{2} \mathrm{Fe}-\mathrm{NHPh}(\mathrm{M})
$$

For interaction energy calculations, the energies of alkali cations $\left(\mathrm{Li}^{+}, \mathrm{Na}^{+}\right.$and $\left.\mathrm{K}^{+}\right)$were obtained in the gas phase with the B3LYP functional and the def2-TZVP basis set, including relativistic effects in zero order regular approximation (ZORA). ${ }^{10-11}$

The natural bond orbital (NBO) analysis of the model complexes 2'-4' were performed using Gaussian 16 at the B3LYP/6-311G(d,p) level of theory based on the ORCA optimized structures. 


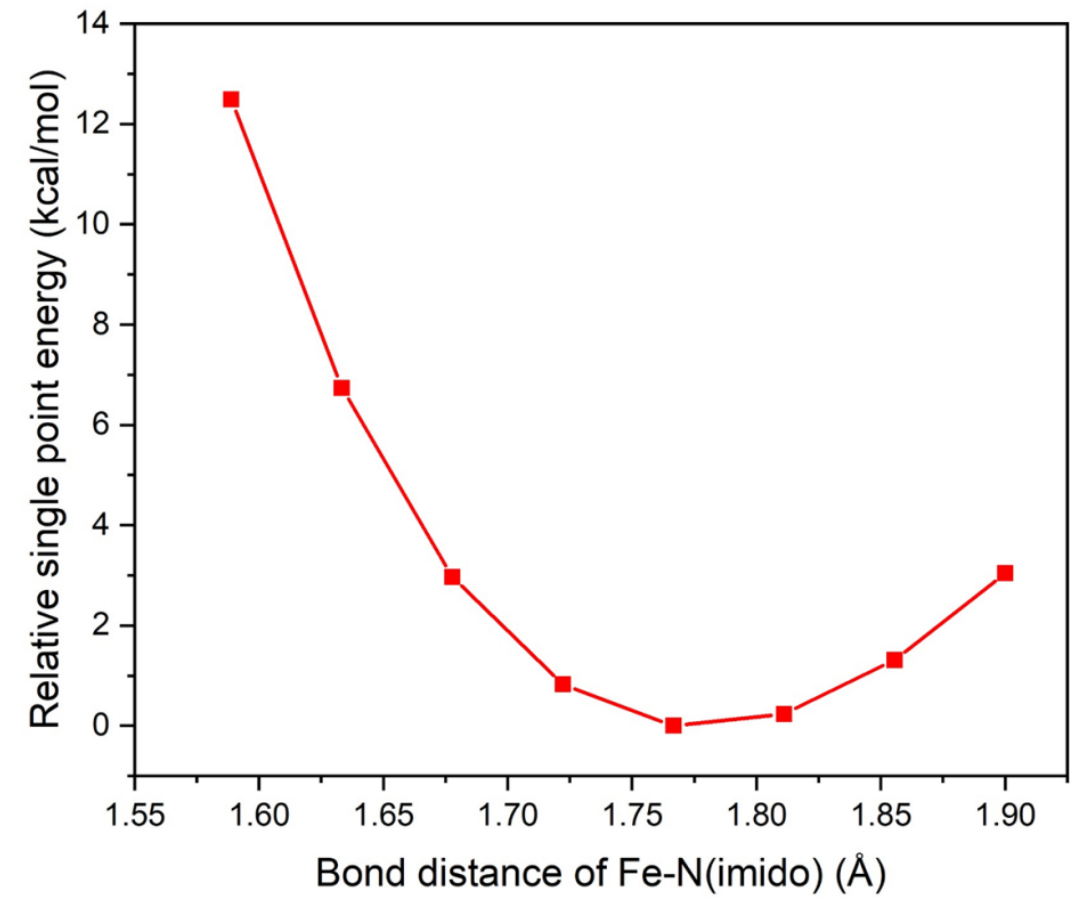

Figure S56. The DFT-calculated single point energy change of $\left[\mathrm{Ph}{ }_{2} \mathrm{~B}\left({ }^{t} \mathrm{BuIm}\right)_{2} \mathrm{Fe}=\mathrm{NDipp}\right]^{-}(S=2)$ (B3LYP/def2-SVP) as a function of Fe-N bond distance.

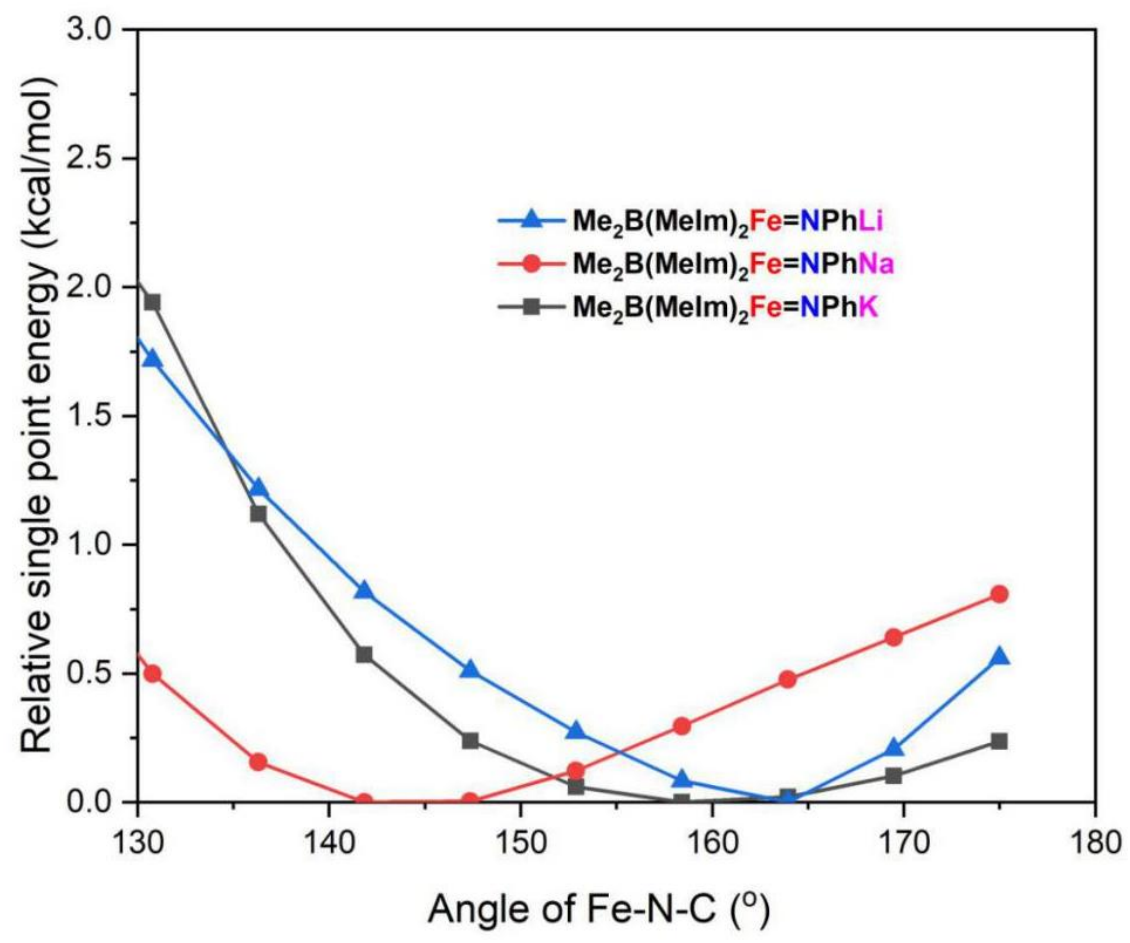

Figure S57. The DFT-calculated single point energy change of the model complexes $\left[\mathrm{Me}_{2} \mathrm{~B}(\mathrm{MeIm})_{2} \mathrm{Fe}=\mathrm{NPhM}\right](\mathrm{M}=\mathrm{Li}, \mathrm{Na}, \mathrm{K})(S=2)(\mathrm{B} 3 \mathrm{LYP} / \mathrm{def} 2-\mathrm{SVP})$ as a function of Fe-N-C angle. 


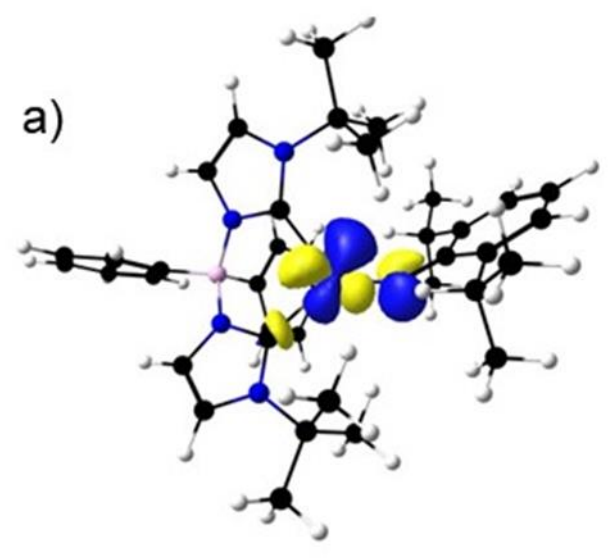

HOMO

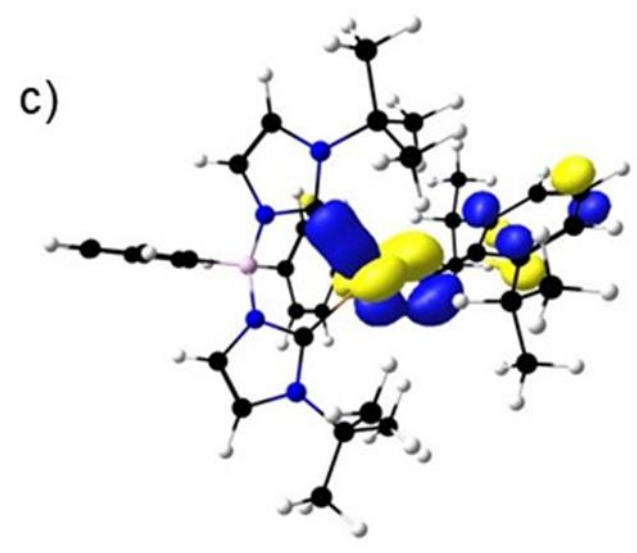

HOMO-5

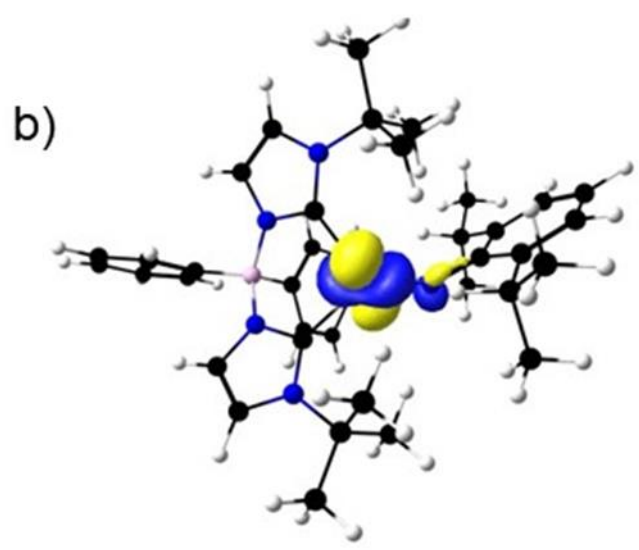

HOMO-1

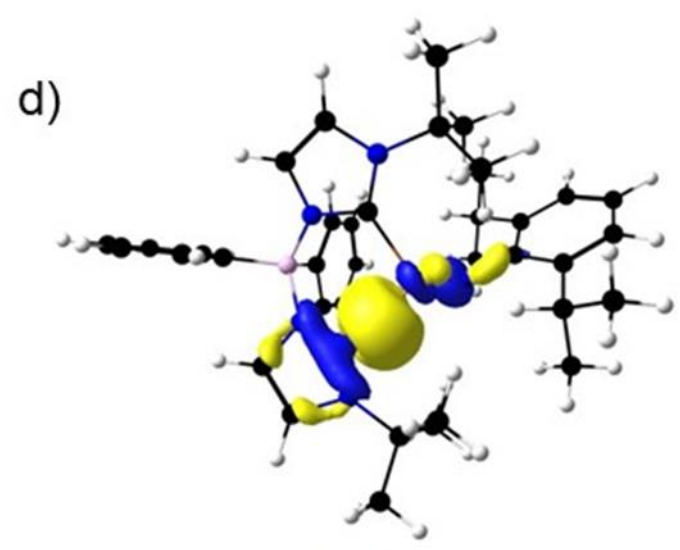

HOMO-7

Figure S58. Selected orbitals showing the $\mathrm{Fe}=\mathrm{N} \quad \sigma$ and $\pi$-interactions in [Ph $\left.{ }_{2} \mathrm{~B}\left({ }^{t} \mathrm{BuIm}\right)_{2} \mathrm{FeNDippLi}\right]\left(\mathbf{2}^{\prime}\right)(S=2)$; (a) $\pi^{*} \mathrm{HOMO}$; (b) $\pi^{*} \mathrm{HOMO}-1$; (c) $\pi$-bonding HOMO-5; (d) $\sigma$-bonding HOMO-7. Natural orbitals shown with isodensity $=0.05$. 
a)

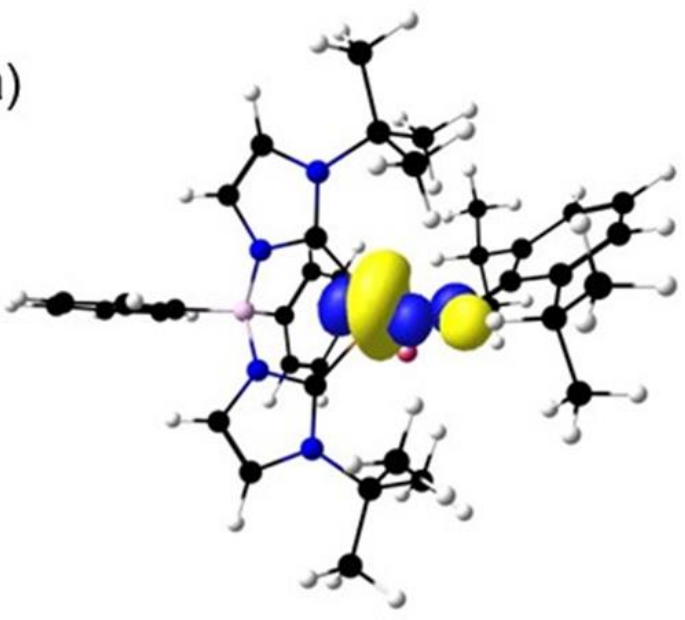

HOMO

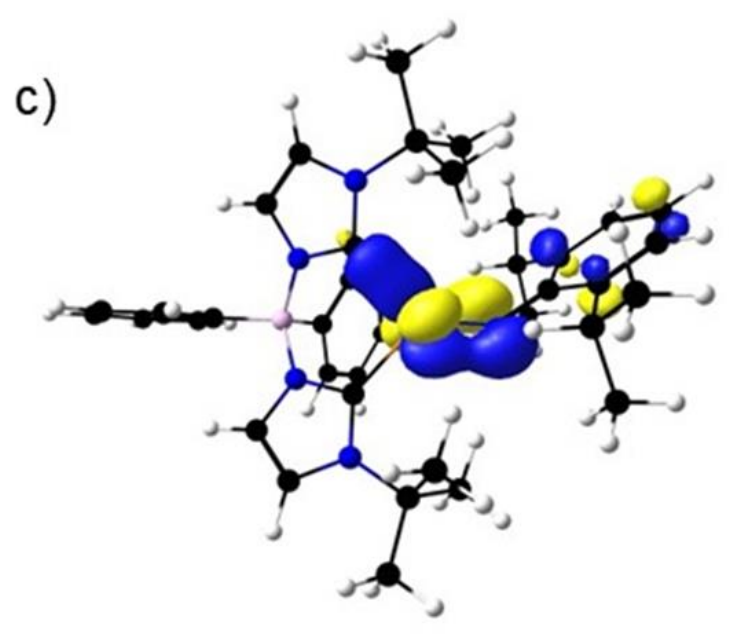

HOMO-5

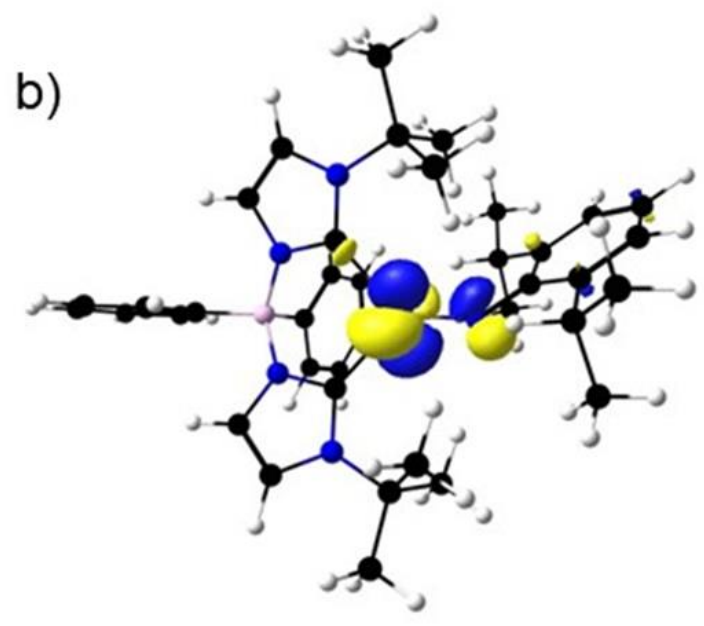

HOMO-1

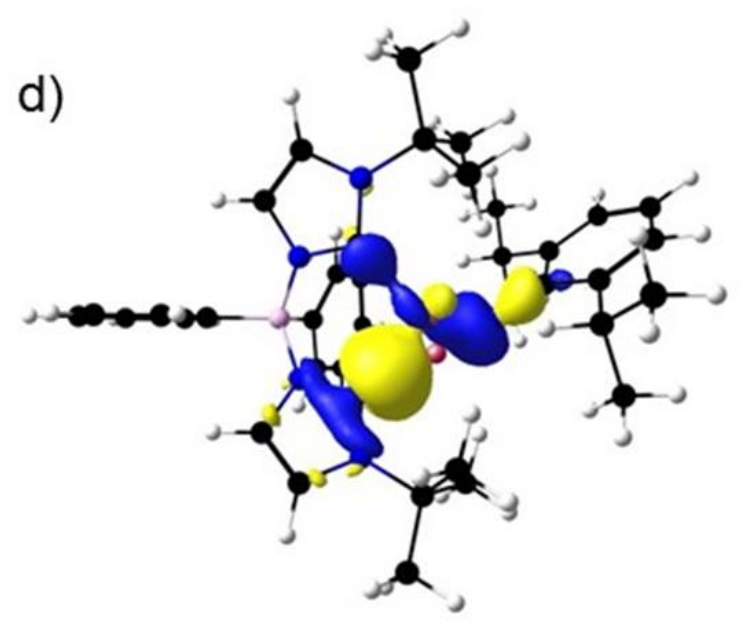

HOMO-7

Figure 559. Selected orbitals showing the $\mathrm{Fe}=\mathrm{N} \quad \sigma$ and $\pi$-interactions in $\left[\mathrm{Ph}_{2} \mathrm{~B}\left({ }^{t} \mathrm{BuIm}\right)_{2} \mathrm{FeNDippNa}\right]\left(3^{\prime}\right)(S=2)$; (a) $\pi^{*} \mathrm{HOMO}$; (b) $\pi^{*} \mathrm{HOMO}-1$; (c) $\pi$-bonding HOMO5; (d) $\sigma$-bonding HOMO-7. Natural orbitals shown with isodensity $=0.05$. 
a)

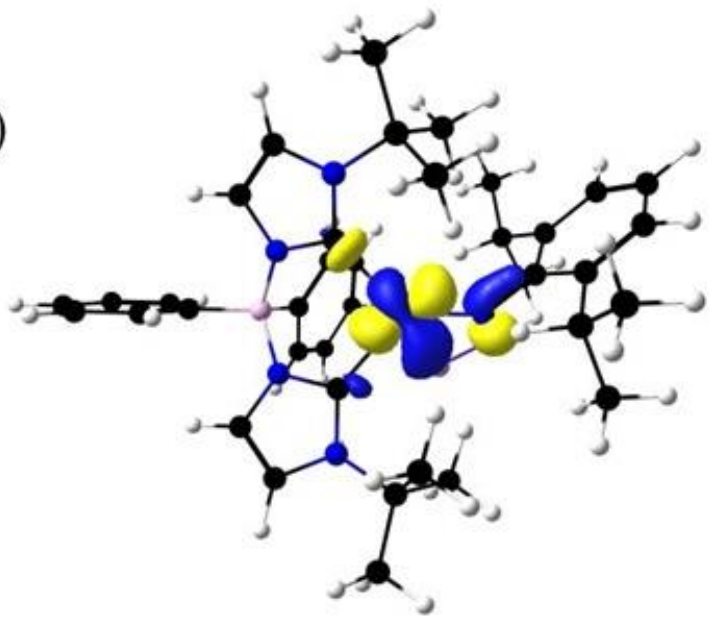

HOMO

c)

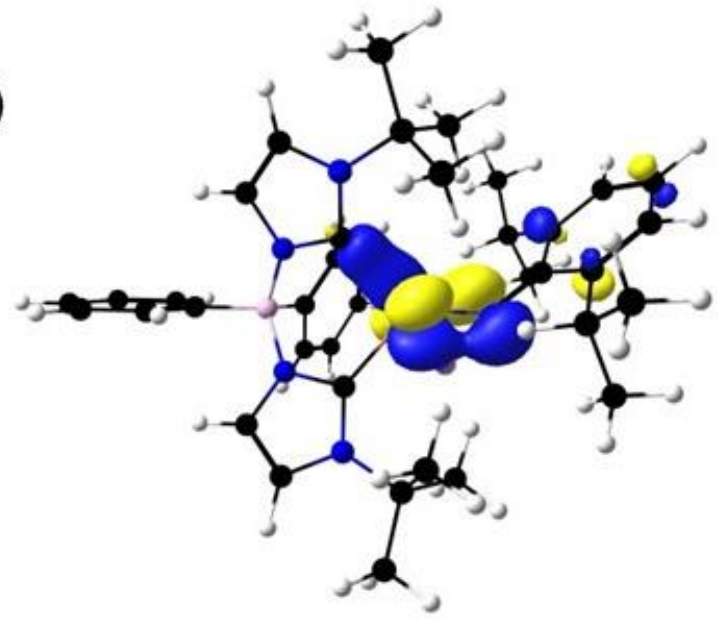

HOMO-5

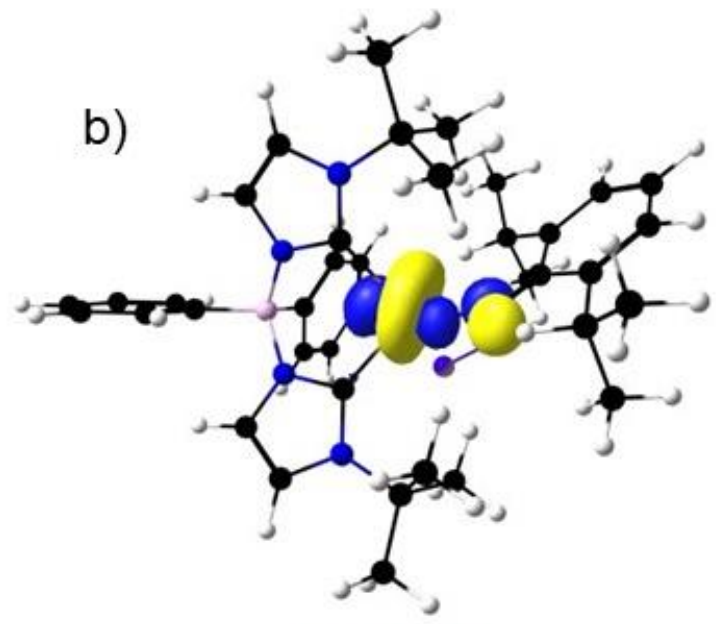

$\mathrm{HOMO}-1$

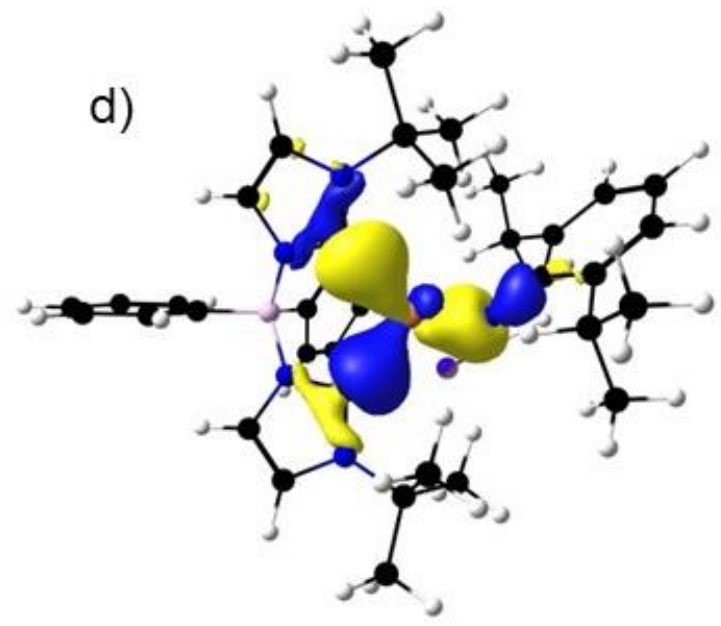

HOMO-7

Figure S60. Selected orbitals showing the $\mathrm{Fe}=\mathrm{N} \sigma$ and $\pi$-interactions in $\left[\mathrm{Ph}_{2} \mathrm{~B}\left({ }^{t} \mathrm{BuIm}\right)_{2} \mathrm{FeNDippK}\right]$ (4') $\left(S=2\right.$ ); (a) $\pi^{*}$ HOMO; (b) $\pi^{*}$ HOMO-1; (c) $\pi$-bonding HOMO-5; (d) $\sigma$-bonding HOMO-7. Natural orbitals shown with isodensity $=0.05$. 
a)

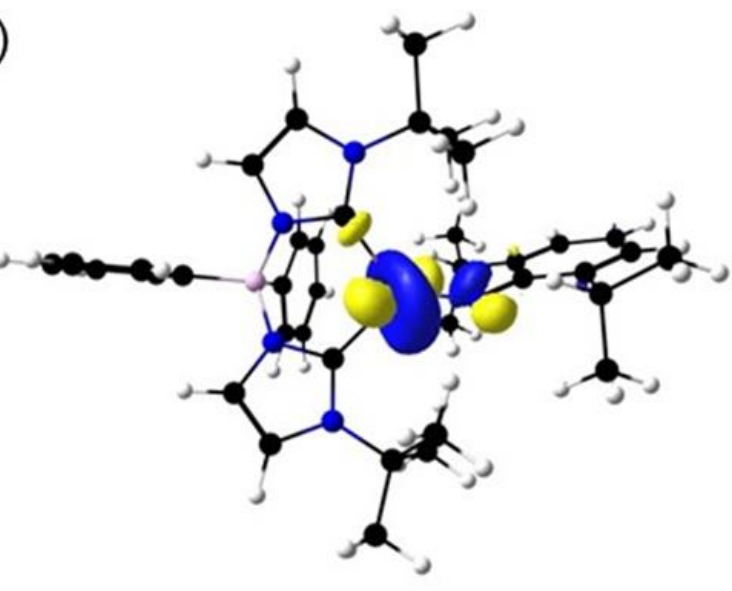

HOMO

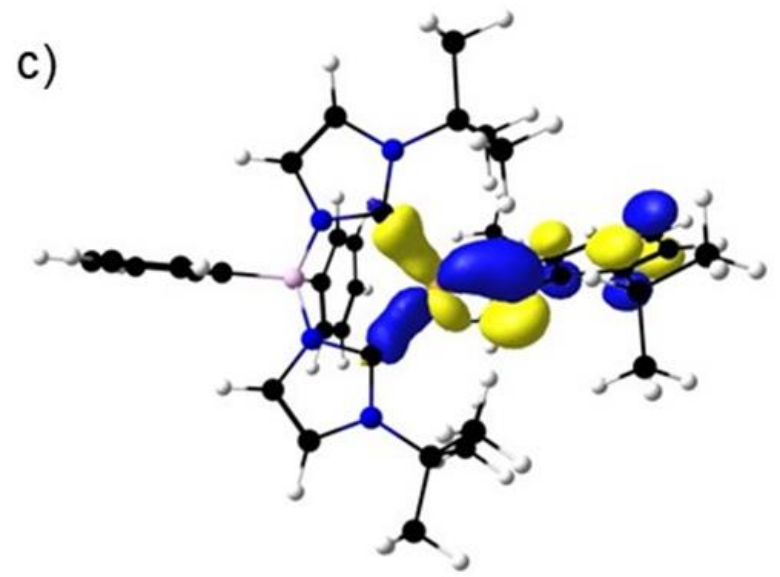

HOMO-6

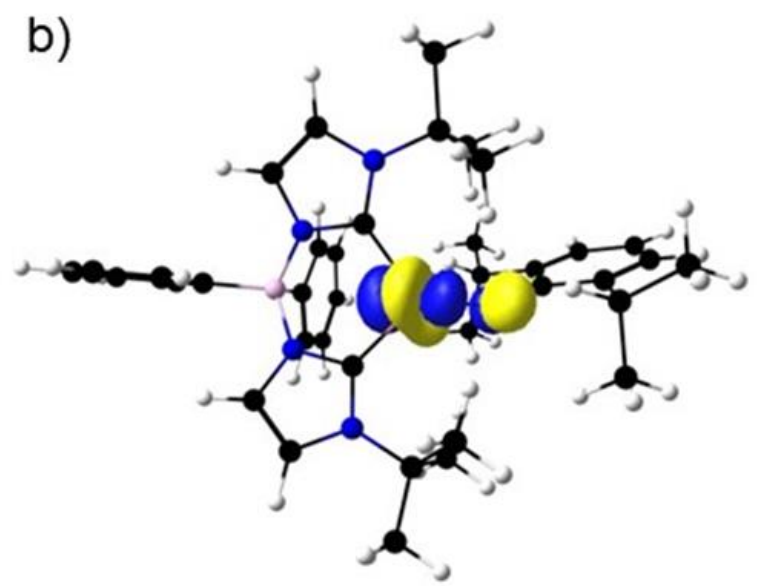

$\mathrm{HOMO}-1$

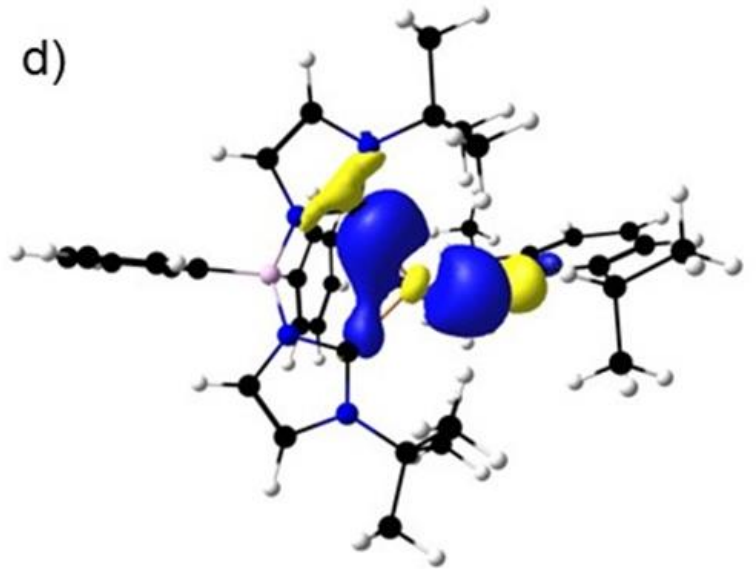

HOMO-7

Figure S61. Selected orbitals showing the $\mathrm{Fe}=\mathrm{N} \sigma$ and $\pi$-interactions in $\left[\mathrm{Ph}_{2} \mathrm{~B}\left({ }^{t} \mathrm{BuIm}\right)_{2} \mathrm{FeNDipp}\right]^{-}$ (5) $(S=2)$; (a) $\pi^{*}$ HOMO; (b) $\pi^{*}$ HOMO-1; (c) $\pi$-bonding HOMO-6; (d) $\sigma$-bonding HOMO-7. Natural orbitals shown with isodensity $=0.05 .^{2}$ Adapted with permission from: Gao, Y.; Carta, V.; Pink, M.; Smith, J. M. Catalytic Carbodiimide Guanylation by a Nucleophilic, High Spin Iron(II) Imido Complex. J. Am. Chem. Soc. 2021, 143, 5324-5329. 


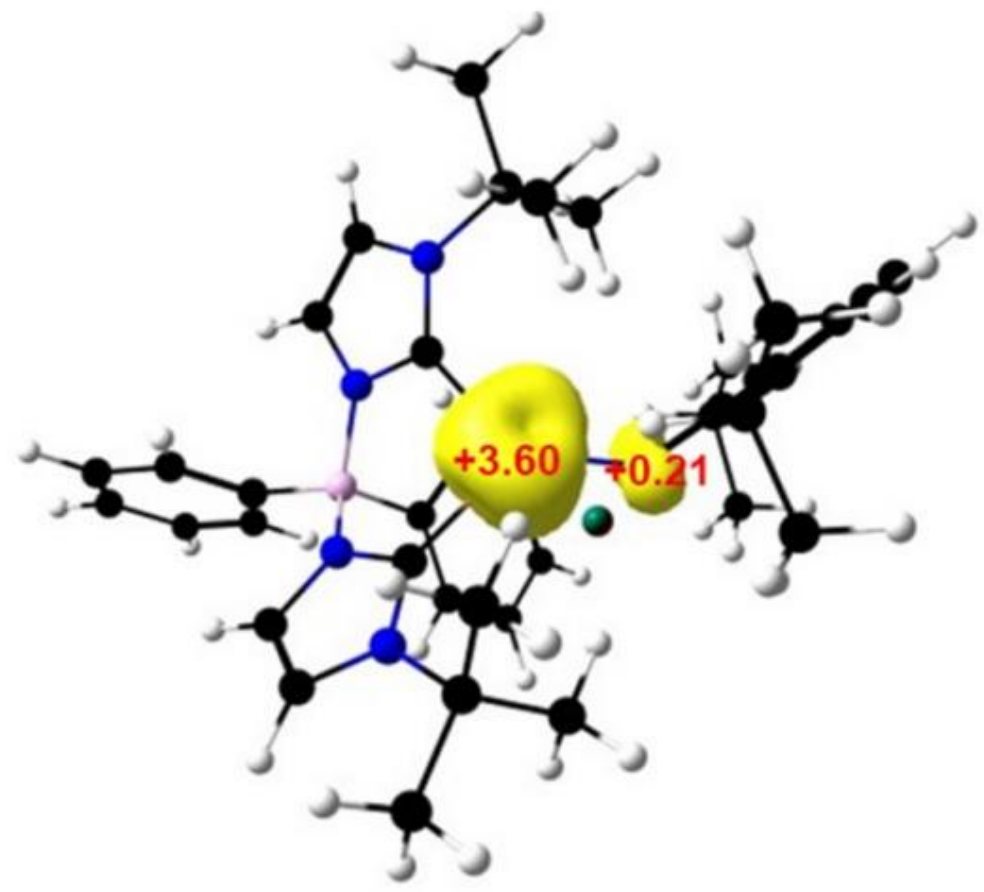

Figure S62. Mulliken atomic spin density distribution of $\left[\mathrm{Ph}_{2} \mathrm{~B}\left({ }^{t} \mathrm{BuIm}\right)_{2} \mathrm{FeNDippLi}\right]\left(\mathbf{2}^{\prime}\right)$ at its $S$ $=2$ state with isodensity $=0.01$.

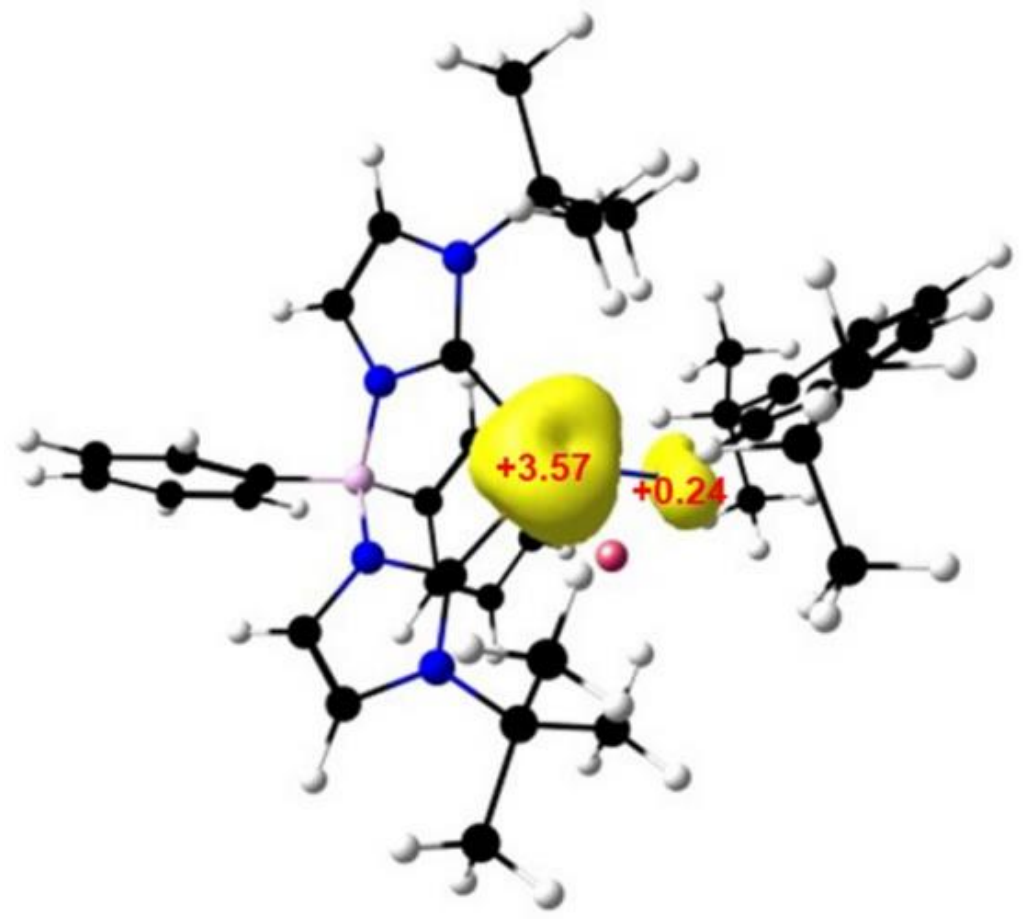

Figure S63. Mulliken atomic spin density distribution of $\left[\mathrm{Ph}{ }_{2} \mathrm{~B}\left({ }^{t} \mathrm{BuIm}\right)_{2} \mathrm{FeNDippNa}\right]\left(\mathbf{3}^{\prime}\right)$ at its $S$ $=2$ state with isodensity $=0.01$. 


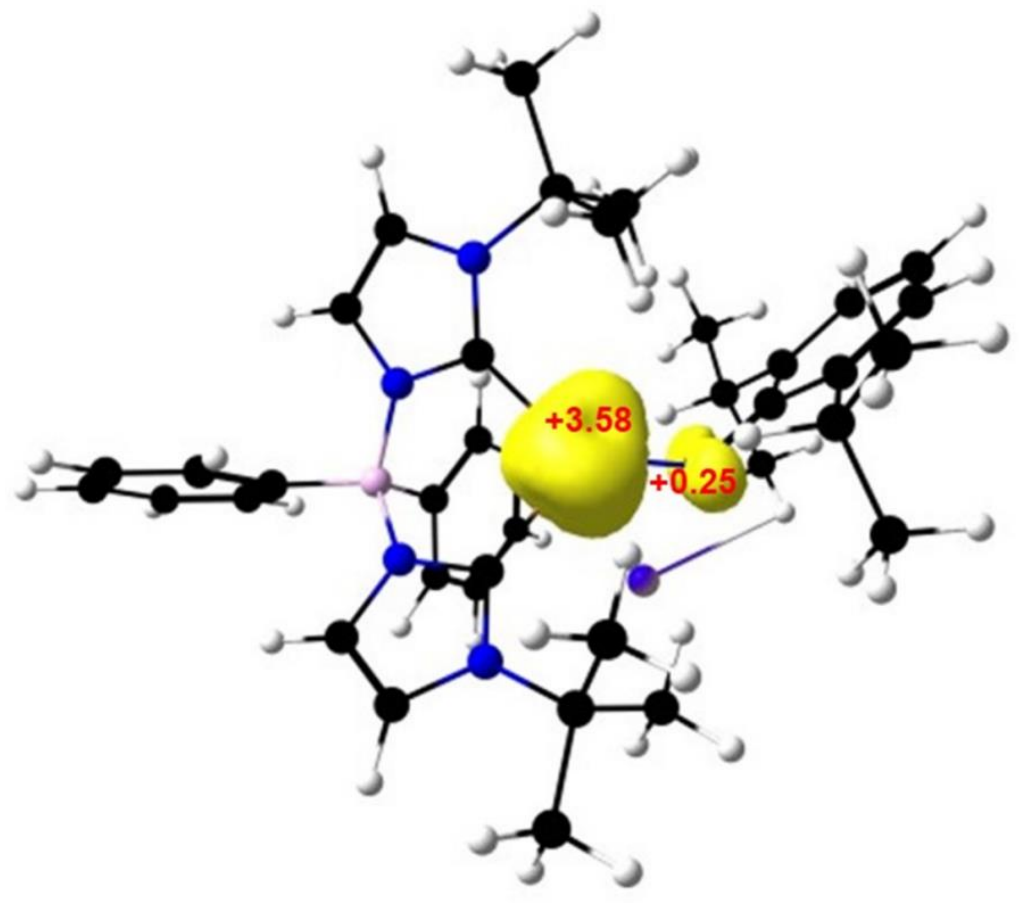

Figure S64. Mulliken atomic spin density distribution of $\left[\mathrm{Ph}_{2} \mathrm{~B}\left({ }^{t} \mathrm{BuIm}\right)_{2} \mathrm{FeNDippK}\right]\left(4^{\prime}\right)$ at its $S=$ 2 state with isodensity $=0.01$.

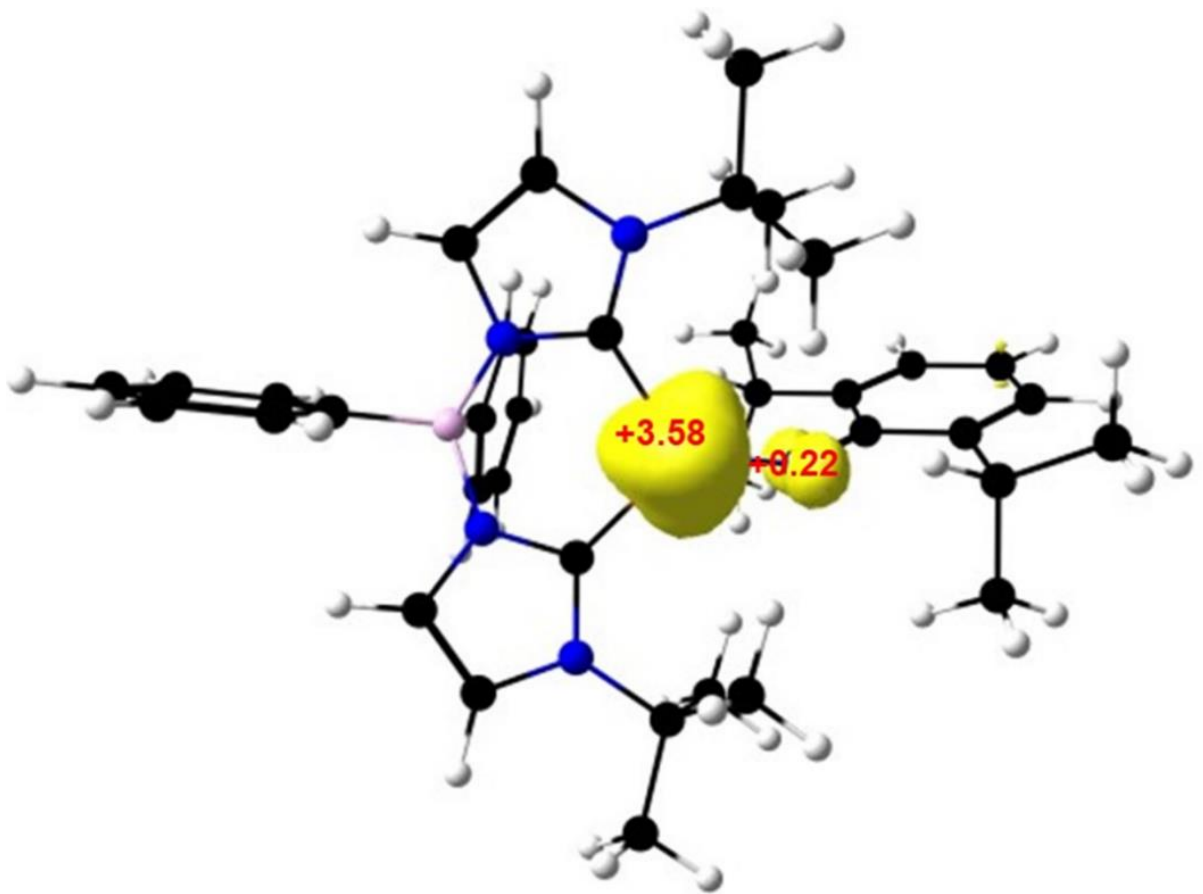

Figure S65. Mulliken atomic spin density distribution of $\left[\mathrm{Ph}_{2} \mathrm{~B}\left({ }^{t} \mathrm{BuIm}\right)_{2} \mathrm{FeNDipp}\right]^{-}(\mathbf{5})$ at its $S=2$ state with isodensity $=0.01 .^{2}$ Reprinted with permission from: Gao, Y.; Carta, V.; Pink, M.; Smith, J. M. Catalytic Carbodiimide Guanylation by a Nucleophilic, High Spin Iron(II) Imido Complex. J. Am. Chem. Soc. 2021, 143, 5324-5329. 


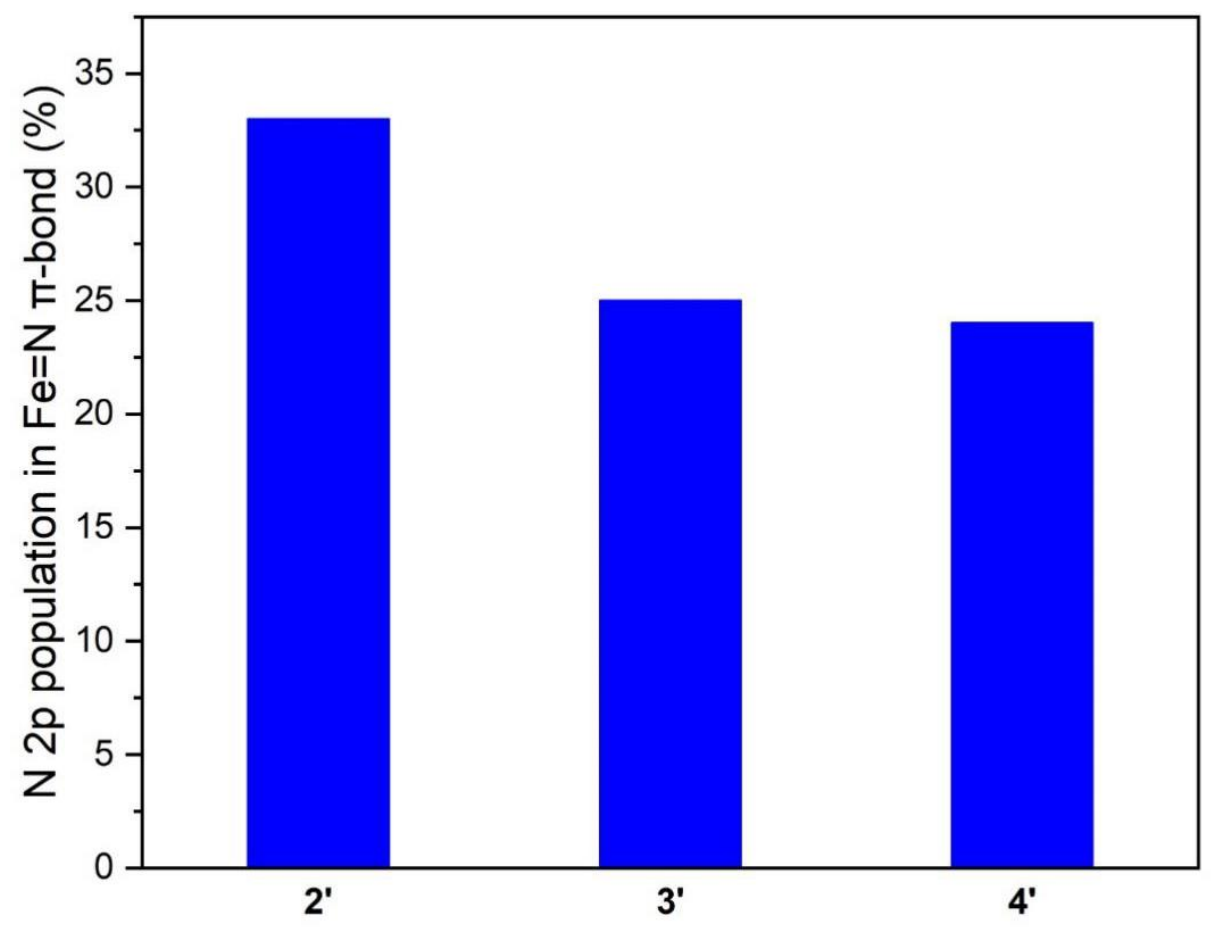

Figure S66. N $2 p$ character in the $\mathrm{Fe}=\mathrm{N} \pi$-bond (HOMO-5) of 2' -4 '. 
Table S1. Selected experimental and calculated (B3LYP/def2-SVP/TZVP) parameters for complexes $\mathbf{2}$ and 3. Calculations were performed both on the full complexes ( 2 and $\mathbf{3})$ and THF free model complexes ( $\boldsymbol{2}^{\prime}$ and $\mathbf{3}^{\prime}$ ).

\begin{tabular}{|c|c|c|c|c|c|c|}
\hline & \multicolumn{3}{|c|}{2} & \multicolumn{3}{|c|}{3} \\
\hline & Exp. & Cal. (2') & Cal. (2) & Exp. & Cal. (3') & Cal. (3) \\
\hline Fe-N(imido) $(\AA)$ & $1.8167(17)$ & 1.829 & 1.815 & $1.812(2)$ & 1.809 & 1.808 \\
\hline $\mathrm{M} \cdots \mathrm{N}($ imido $)(\AA)^{a}$ & $1.950(4)$ & 1.869 & 1.880 & $2.440(3)$ & 2.279 & 2.308 \\
\hline $\mathrm{Fe}-\mathrm{C}(\AA)^{b}$ & $2.110(2)$ & 2.085 & 2.085 & $2.097(3)$ & 2.081 & 2.092 \\
\hline $\mathrm{Fe}-\mathrm{N}-\mathrm{C}\left(\left(^{\mathrm{o}}\right)\right.$ & $143.59(14)$ & 132.7 & 139.6 & $141.5(2)$ & 135.2 & 142.6 \\
\hline
\end{tabular}

${ }^{a} \mathrm{M}=\mathrm{Li}$ or $\mathrm{Na} ;{ }^{b}$ Average of the $\mathrm{Fe}-\mathrm{C}($ carbene) bond distances.

Table S2. Selected parameters for complexes $\mathbf{2}$ and $\mathbf{3}$ obtained from density functional theory calculations ((B3LYP/def2-SVP/TZVP). Calculations were performed both on the full complexes ( 2 and $\mathbf{3}$ ) and THF free model complexes ( ${ }^{\prime}$ ' and $\mathbf{3}^{\prime}$ ).

\begin{tabular}{cccccc}
\hline & & $\mathbf{2}^{\prime}$ & $\mathbf{2}$ & $\mathbf{3}^{\prime}$ & $\mathbf{3}$ \\
\hline Mulliken spin & $\mathrm{Fe}$ & 3.60 & 3.64 & 3.57 & 3.63 \\
density & $\mathrm{N}$ & 0.21 & 0.21 & 0.24 & 0.21 \\
\hline $\begin{array}{c}\text { Löwdin bond } \\
\text { order }\end{array}$ & & 1.14 & 1.29 & 1.17 & 1.29 \\
\hline
\end{tabular}


Table S3. Summary of NBO analysis.

\begin{tabular}{|c|c|c|c|c|}
\hline Complex & $\mathrm{c}_{\mathrm{A}}^{2}(\%)$ & Hybridization & Assignment & $\begin{array}{l}\text { Wiberg } \\
\text { bond } \\
\text { index }\end{array}$ \\
\hline \multirow[t]{4}{*}[\mathrm{Ph}{}_{2}\mathrm{B}({}^{t}\mathrm{BuIm})_{2}\mathrm{Fe}=\mathrm{NDippLi}]{$\left(\mathbf{2}^{\prime}\right)$} & 17.60 & Fe $8.19 \% s, 0.66 \% p, 91.11 \% d, 0.04 \% f$ & $\pi$ & \\
\hline & 82.40 & $\mathrm{~N} 6.60 \% s, 93.38 \% p, 0.01 \% d$ & & 0.47 \\
\hline & 18.49 & $\mathrm{Fe} 5.09 \% s, 0.40 \% p, 94.49 \% d, 0.02 \% f$ & & \\
\hline & 81.51 & $\mathrm{~N} 0.88 \% s, 99.10 \% p, 0.02 \% \mathrm{~d}$ & & \\
\hline \multirow[t]{3}{*}[\mathrm{Ph}_{2}\mathrm{B}({}^{t}\mathrm{BuIm})_{2}\mathrm{Fe}=\mathrm{NDippNa}]{$\left(\mathbf{3}^{\prime}\right)$} & 17.97 & Fe $1.36 \% s, 0.86 \% p, 97.76 \% d, 0.03 \% f$ & $\pi$ & \\
\hline & 82.03 & $\mathrm{~N} 1.44 \% s, 98.55 \% p, 0.02 \% d$ & & 0.51 \\
\hline & $\begin{array}{l}21.01 \\
78.99\end{array}$ & $\begin{array}{l}\text { Fe } 7.92 \% s, 0.31 \% p, 91.74 \% d, 0.02 \% f \\
\text { N } 1.93 \% s, 98.05 \% p, 0.02 \% d\end{array}$ & $\pi$ & \\
\hline \multirow[t]{3}{*}[\mathrm{Ph}_{2}\mathrm{B}({}^{t}\mathrm{BuIm})_{2}\mathrm{Fe}=\mathrm{NDippK}]{$\left(4^{\prime}\right)$} & 19.38 & $\mathrm{Fe} 0.51 \% s, 0.59 \% p, 98.88 \% d, 0.02 \% f$ & $\pi$ & \\
\hline & 80.62 & $\mathrm{~N} 0.56 \% s, 99.42 \% p, 0.02 \% d$ & & 0.52 \\
\hline & $\begin{array}{l}20.66 \\
79.34\end{array}$ & $\begin{array}{l}\text { Fe } 8.06 \% s, 0.30 \% p, 91.61 \% d, 0.03 \% f \\
\text { N } 2.30 \% s, 97.67 \% p, 0.03 \% d\end{array}$ & $\pi$ & \\
\hline \multirow[t]{3}{*}[\mathrm{Ph}_{2}\mathrm{B}({}^{(}\mathrm{BuIm})_{2}\mathrm{Fe}=\mathrm{NDipp}]{$^{-}(\mathbf{5})$} & 24.96 & $\mathrm{Fe} 0.12 \% s, 0.48 \% p, 99.38 \% d, 0.02 \% f$ & $\pi$ & \\
\hline & 75.04 & $\mathrm{~N} 0.62 \% s, 99.37 \% p, 0.02 \% d$ & & 0.55 \\
\hline & 16.55 & $\begin{array}{l}\text { Fe } 3.04 \% s, 0.54 \% p, 96.39 \% d, 0.03 \% f \\
\text { N } 0.87 \% s, 99.11 \% p, 0.02 \% d\end{array}$ & $\pi$ & \\
\hline
\end{tabular}




\section{Crystallographic Information}

\section{$\left.\left[\mathrm{Ph}_{2} \underline{\mathrm{B}\left({ }^{t} \mathrm{BuIm}\right)_{2}} \underline{\mathrm{FeNDippLi}} \mathrm{THF}\right)_{2}\right](\mathbf{2})$}

\section{Data collection}

The data collection was carried out using Mo Ka radiation (graphite monochromator) with a frame time of 1 seconds and a detector distance of $4.00 \mathrm{~cm}$. A collection strategy was calculated and complete data to a resolution of $0.77 \AA$ with a redundancy of 10 were collected. Eleven major sections of frames were collected with $1^{\circ}$ and scans. A total of 1942 frames were collected. The total exposure time was 1.42 hours. The frames were integrated with the Bruker SAINT software package $^{14}$ using a narrow-frame algorithm. The integration of the data using a triclinic unit cell yielded a total of 124535 reflections to a maximum $\theta$ angle of $27.51^{\circ}(0.77 \AA$ resolution $)$, of which 11431 were independent (average redundancy 10.894, completeness $=99.9 \%$, Rint $=10.51 \%$, Rsig $=5.50 \%)$ and $8108(70.93 \%)$ were greater than $2 \sigma\left(\mathrm{F}^{2}\right)$. The final cell constants of $\mathrm{a}=11.2028(5)$ $\AA, b=15.4632(6) \AA, c=16.2337(6) \AA, \alpha=70.3936(12)^{\circ}, \beta=75.8923(13)^{\circ}, \gamma=71.6395(13)^{\circ}$, volume $=2484.12(18) \AA^{3}$, are based upon the refinement of the XYZ-centroids of 9438 reflections above $20 \sigma(\mathrm{I})$ with $5.053^{\circ}<2 \theta<53.27^{\circ}$. Data were corrected for absorption effects using the Multi-Scan method (SADABS). ${ }^{15}$ The ratio of minimum to maximum apparent transmission was 0.855. The calculated minimum and maximum transmission coefficients (based on crystal size) are 0.9060 and 0.9920 .

\section{Structure solution and refinement}

The space group P-1 was determined based on intensity statistics and systematic absences. The structure was solved and refined using the SHELX suite of programs. ${ }^{16-17}$ An intrinsicmethods solution was calculated, which provided most non-hydrogen atoms from the E-map. Fullmatrix least squares / difference Fourier cycles were performed, which located the remaining nonhydrogen atoms. All non-hydrogen atoms were refined with anisotropic displacement parameters. The hydrogen atoms were placed in ideal positions and refined as riding atoms with relative isotropic displacement parameters. The final anisotropic full-matrix least-squares refinement on $\mathrm{F}^{2}$ with 562 variables converged at $\mathrm{R} 1=4.84 \%$, for the observed data and $\mathrm{wR} 2=11.88 \%$ for all data. The goodness-of-fit was 1.042. The largest peak in the final difference electron density synthesis was $0.422 \mathrm{e}^{-} / \AA^{3}$ and the largest hole was $-0.417 \mathrm{e}^{-} / \AA^{3}$ with an RMS deviation of $0.056 \mathrm{e}^{-}$ $/ \AA^{3}$. On the basis of the final model, the calculated density was $1.157 \mathrm{~g} / \mathrm{cm}^{3}$ and $\mathrm{F}(000), 936 \mathrm{e}^{-}$. 
Table S4. Crystal data and structure refinement for $\left[\mathrm{Ph}{ }_{2} \mathrm{~B}\left({ }^{t} \mathrm{BuIm}\right)_{2} \mathrm{FeNDippLi}(\mathrm{THF})_{2}\right](2)$.

Empirical formula

Formula weight

Crystal color, shape, size

Temperature

Wavelength

Crystal system, space group

Unit cell dimensions

Volume

$\mathrm{Z}$

Density (calculated)

Absorption coefficient

$\mathrm{F}(000)$

\section{Data collection}

Diffractometer

Theta range for data collection

Index ranges

Reflections collected

Independent reflections

Observed Reflections

Completeness to theta $=25.242^{\circ}$

\section{Solution and Refinement}

Absorption correction

Max. and min. transmission

Solution

Refinement method

Weighting scheme

Data / restraints / parameters

Goodness-of-fit on $\mathrm{F}^{2}$

Final R indices [I $>2 \operatorname{sigma}(\mathrm{I})]$

$\mathrm{R}$ indices (all data)

Largest diff. peak and hole
C51 H77 B Fe Li N5 O2

865.77

red plate, $0.291 \times 0.111 \times 0.022 \mathrm{~mm}^{3}$

153(2) K

$0.71073 \AA$

Triclinic, P-1

$\mathrm{a}=11.2028(5) \AA$

$\alpha=70.3936(12)^{\circ}$.

$\mathrm{b}=15.4632(6) \AA$

$\mathrm{c}=16.2337(6) \AA$

2484.12(18) $\AA^{3}$

2

$1.157 \mathrm{Mg} / \mathrm{m}^{3}$

$0.346 \mathrm{~mm}^{-1}$

936

Venture D8, Bruker

1.939 to $27.513^{\circ}$.

$-14<=\mathrm{h}<=14,-20<=\mathrm{k}<=20,-21<=\mathrm{l}<=21$

124535

$11431\left[\mathrm{R}_{\mathrm{int}}=0.1051\right]$

8108

$100.0 \%$

Semi-empirical from equivalents

0.7456 and 0.6372

Intrinsic methods

Full-matrix least-squares on $\mathrm{F}^{2}$

$\mathrm{w}=\left[\sigma^{2} \mathrm{Fo}^{2}+\mathrm{AP}^{2}+\mathrm{BP}\right]^{-1}$, with

$\mathrm{P}=\left(\mathrm{Fo}^{2}+2 \mathrm{Fc}^{2}\right) / 3, \mathrm{~A}=0.0410, \mathrm{~B}=1.5152$

$11431 / 0 / 562$

1.043

$\mathrm{R} 1=0.0484, \mathrm{wR} 2=0.1033$

$\mathrm{R} 1=0.0838, \mathrm{wR} 2=0.1187$

0.422 and -0.416 e. $\AA^{-3 \square}$ 


\section{$\left[\mathrm{Ph}_{2} \mathrm{~B}\left({ }^{t} \mathrm{BuIm}\right)_{2} \mathrm{FeNDippNa}(\mathrm{THF})_{3}\right](\mathbf{3})$}

\section{Data collection}

The data collection was carried out using Mo K $\alpha$ radiation (graphite monochromator) with a frame time of 1 second and a detector distance of $4.50 \mathrm{~cm}$. A collection strategy was calculated and complete data to a resolution of $0.77 \AA$ with a redundancy of 25 were collected. Eleven major sections of frames were collected with $1^{\circ} \omega$ and $\varphi$ scans. A total of 1617 frames were collected. The total exposure time was 3.64 hours. The frames were integrated with the Bruker SAINT software package ${ }^{14}$ using a narrow-frame algorithm. The integration of the data using an orthorhombic unit cell yielded a total of 162585 reflections to a maximum $\theta$ angle of $27.56^{\circ}(0.77$ $\AA$ resolution), of which 11292 were independent (average redundancy 14.398, completeness = $99.7 \%$, Rint $=8.05 \%$, Rsig $=3.44 \%)$ and $9417(83.40 \%)$ were greater than $2 \sigma\left(\mathrm{F}^{2}\right)$. The final cell

constants of $\mathrm{a}=12.240(2) \AA, \mathrm{b}=16.139(4) \AA, \mathrm{c}=24.831(5) \AA$, volume $=4905.1(17) \AA^{3}$, are based upon the refinement of the XYZ-centroids of 9023 reflections above $20 \sigma(\mathrm{I})$ with $5.308^{\circ}<$ $2 \theta<51.65^{\circ}$. Data were corrected for absorption effects using the Multi-Scan method (SADABS). ${ }^{15}$ The ratio of minimum to maximum apparent transmission was 0.917 . The calculated minimum and maximum transmission coefficients (based on crystal size) are 0.8810 and 0.9710 .

\section{Structure solution and refinement}

The space group P $2{ }_{1} 2{ }_{1} 2_{1}$ was determined based on intensity statistics and systematic absences. The structure was solved and refined using the SHELX suite of programs. ${ }^{16-17}$ An intrinsicmethods solution was calculated, which provided most non-hydrogen atoms from the E-map. Fullmatrix least squares / difference Fourier cycles were performed, which located the remaining nonhydrogen atoms. All non-hydrogen atoms were refined with anisotropic displacement parameters. The hydrogen atoms were placed in ideal positions and refined as riding atoms with relative isotropic displacement parameters. Disorder was refined for two thf and the isopropylaniline moieties; sets of restraints and constraints were applied. The final anisotropic full-matrix leastsquares refinement on $\mathrm{F}^{2}$ with 628 variables converged at $\mathrm{R} 1=3.93 \%$, for the observed data and $\mathrm{wR} 2=9.24 \%$ for all data. The goodness-of-fit was 1.050 . The largest peak in the final difference electron density synthesis was $0.289 \mathrm{e}^{-} / \AA^{3}$ and the largest hole was $-0.274 \mathrm{e}^{-} / \AA^{3}$ with an RMS deviation of $0.048 \mathrm{e}^{-} / \AA^{3}$. On the basis of the final model, the calculated density was $1.194 \mathrm{~g} / \mathrm{cm}^{3}$ and $\mathrm{F}(000), 1896 \mathrm{e}^{-}$. 
Table S5. Crystal data and structure refinement for $\left[\mathrm{Ph}{ }_{2} \mathrm{~B}\left({ }^{t} \mathrm{BuIm}\right)_{2} \mathrm{FeNDippNa}(\mathrm{THF})_{3}\right](3)$.

Empirical formula

Formula weight

Crystal color, shape, size

Temperature

Wavelength

Crystal system, space group

Unit cell dimensions

Volume

$\mathrm{Z}$

Density (calculated)

Absorption coefficient

$\mathrm{F}(000)$

\section{Data collection}

Diffractometer

Theta range for data collection

Index ranges

Reflections collected

Independent reflections

Observed Reflections

Completeness to theta $=25.242^{\circ}$

Solution and Refinement

Absorption correction

Max. and min. transmission

Solution

Refinement method

Weighting scheme

Data / restraints / parameters

Goodness-of-fit on $\mathrm{F}^{2}$

Final R indices [I $>2 \operatorname{sigma}(\mathrm{I})]$

$\mathrm{R}$ indices (all data)

Largest diff. peak and hole
C50 H73 B Fe N5 Na O3

881.78

red plate, $0.361 \times 0.264 \times 0.082 \mathrm{~mm}^{3}$

153(2) K

$0.71073 \AA$

Orthorhombic, $\mathrm{P} 2{ }_{1} 2{ }_{1} 2_{1}$

$\mathrm{a}=12.240(2) \AA$ $\alpha=90^{\circ}$.

$\mathrm{b}=16.139(4) \AA$

$\mathrm{c}=24.831(5) \AA$

4905.1(17) $\AA^{3}$

4

$1.194 \mathrm{Mg} / \mathrm{m}^{3}$

$0.361 \mathrm{~mm}^{-1}$

1896

Venture D8, Bruker

1.855 to $27.557^{\circ}$.

$-15<=\mathrm{h}<=15,-20<=\mathrm{k}<=21,-32<=\mathrm{l}<=32$

162585

$11292\left[\mathrm{R}_{\mathrm{int}}=0.0805\right]$

9417

$99.9 \%$

Semi-empirical from equivalents

0.7325 and 0.6628

Intrinsic methods

Full-matrix least-squares on $\mathrm{F}^{2}$

$\mathrm{w}=\left[\sigma^{2} \mathrm{Fo}^{2}+\mathrm{AP}^{2}+\mathrm{BP}\right]^{-1}$, with

$\mathrm{P}=\left(\mathrm{Fo}^{2}+2 \mathrm{Fc}^{2}\right) / 3, \mathrm{~A}=0.0345, \mathrm{~B}=1.6099$

11292 / 349 / 628

1.050

$\mathrm{R} 1=0.0393, \mathrm{wR} 2=0.0831$

$\mathrm{R} 1=0.0562, \mathrm{wR} 2=0.0924$

0.289 and -0.274 e. $\AA^{-3}$

$\left[\mathrm{Ph}_{2} \underline{\mathrm{B}}^{\left({ }^{t} \mathrm{BuIm}\right)}{ }_{2} \underline{\mathrm{FeNDippK}}_{2} \underline{(4)}\right.$ 


\section{Data collection}

The data collection was carried out using Mo K $\alpha$ radiation (graphite monochromator) with a frame time of 3 seconds and a detector distance of $5.00 \mathrm{~cm}$. A collection strategy was calculated and complete data to a resolution of $0.77 \AA$ with a redundancy of 3.4 were collected. Five major sections of frames were collected with $0.50^{\circ} \omega$ and $\varphi$ scans. A total of 1878 frames were collected. The total exposure time was 1.47 hours. The frames were integrated with the Bruker SAINT software package ${ }^{14}$ using a narrow-frame algorithm. The integration of the data using a monoclinic unit cell yielded a total of 173867 reflections to a maximum $\theta$ angle of $25.70^{\circ}$ ( $0.82 \AA$ resolution), of which 13742 were independent (average redundancy 12.652, completeness $=99.9 \%, \mathrm{R}_{\text {int }}=$ $\left.16.81 \%, \mathrm{R}_{\text {sig }}=7.03 \%\right)$ and $8939(65.05 \%)$ were greater than $2 \sigma\left(\mathrm{F}^{2}\right)$. The final cell constants of a $=15.7047(7) \AA, b=34.0372(11) \AA, c=13.5154(6) \AA, \beta=90.6804(15)^{\circ}$, volume $=7224.1(5) \AA^{3}$, are based upon the refinement of the XYZ-centroids of 5696 reflections above $20 \sigma(\mathrm{I})$ with $4.661^{\circ}$ $<2 \theta<42.34^{\circ}$. Data were corrected for absorption effects using the Multi-Scan method (SADABS). ${ }^{15}$ The ratio of minimum to maximum apparent transmission was 0.956 . The calculated minimum and maximum transmission coefficients (based on crystal size) are 0.9350 and 0.9610 .

\section{Structure solution and refinement}

The space group $\mathrm{P} 2{ }_{1} / \mathrm{c}$ was determined based on intensity statistics and systematic absences. The structure was solved and refined using the SHELX suite of programs. ${ }^{16-17}$ An intrinsicmethods solution was calculated, which provided most non-hydrogen atoms from the E-map. Fullmatrix least squares / difference Fourier cycles were performed, which located the remaining nonhydrogen atoms. All non-hydrogen atoms were refined with anisotropic displacement parameters. The hydrogen atoms were placed in ideal positions and refined as riding atoms with relative isotropic displacement parameters. The final anisotropic full-matrix least-squares refinement on $\mathrm{F}^{2}$ with 849 variables converged at $\mathrm{R} 1=5.60 \%$, for the observed data and $\mathrm{wR} 2=14.82 \%$ for all data. The goodness-of-fit was 1.026. The largest peak in the final difference electron density synthesis was $0.438 \mathrm{e}^{-} / \AA^{3}$ and the largest hole was $-0.666 \mathrm{e}^{-} / \AA^{3}$ with an RMS deviation of $0.088 \mathrm{e}^{-}$ $/ \AA^{3}$. On the basis of the final model, the calculated density was $1.253 \mathrm{~g} / \mathrm{cm}^{3}$ and $\mathrm{F}(000), 2896 \mathrm{e}^{-}$. Two half molecules are in the asymmetric unit. 
Table S6. Crystal data and structure refinement for $\left[\mathrm{Ph}_{2} \mathrm{~B}\left({ }^{t} \mathrm{BuIm}\right)_{2} \mathrm{FeNDippK}\right]_{2}$ (4).

Empirical formula

Formula weight

Crystal color, shape, size

Temperature

Wavelength

Crystal system, space group

Unit cell dimensions

Volume

Z

Density (calculated)

Absorption coefficient

$\mathrm{F}(000)$

\section{Data collection}

Diffractometer

Theta range for data collection

Index ranges

Reflections collected

Independent reflections

Observed Reflections

Completeness to theta $=25.242^{\circ}$

\section{Solution and Refinement}

Absorption correction

Max. and min. transmission

Solution

Refinement method

Weighting scheme

Data / restraints / parameters

Goodness-of-fit on $\mathrm{F}^{2}$

Final $\mathrm{R}$ indices [I>2sigma(I)]

$\mathrm{R}$ indices (all data)

Largest diff. peak and hole
C76 H98 B2 Fe2 K2 N10

1363.16

red block, $0.12 \times 0.07 \times 0.07 \mathrm{~mm}^{3}$

100(2) K

$0.71073 \AA$

Monoclinic, $\mathrm{P} 2{ }_{1} / \mathrm{c}$

$\mathrm{a}=15.7047(7) \AA \quad \alpha=90^{\circ}$.

$\mathrm{b}=34.0372(11) \AA \quad \beta=90.6804(15)^{\circ}$.

$\mathrm{c}=13.5154(6) \AA \quad \gamma=90^{\circ}$.

7224.1(5) $\AA^{3}$

4

$1.253 \mathrm{Mg} / \mathrm{m}^{3}$

$0.566 \mathrm{~mm}^{-1}$

2896

Venture D8, Bruker

1.924 to $25.696^{\circ}$.

$-19<=\mathrm{h}<=19,-41<=\mathrm{k}<=41,-16<=1<=16$

173867

$13742\left[\mathrm{R}_{\text {int }}=0.1681\right]$

8939

$100.0 \%$

Semi-empirical from equivalents

0.7243 and 0.6927

Intrinsic methods

Full-matrix least-squares on $\mathrm{F}^{2}$

$\mathrm{w}=\left[\sigma^{2} \mathrm{Fo}^{2}+\mathrm{AP}^{2}+\mathrm{BP}\right]^{-1}$, with

$\mathrm{P}=\left(\mathrm{Fo}^{2}+2 \mathrm{Fc}^{2}\right) / 3, \mathrm{~A}=0.0492, \mathrm{~B}=14.4844$

13742 / 0 / 849

1.026

$\mathrm{R} 1=0.0560, \mathrm{wR} 2=0.1182$

$\mathrm{R} 1=0.1021, \mathrm{wR} 2=0.1482$

0.438 and -0.666 e. $\AA^{-3 \square}$ 


\section{$\underline{\mathrm{Ph}}_{2} \underline{\mathrm{B}\left({ }^{t} \mathrm{BuIm}\right)_{2}} \underline{\mathrm{FeNHDipp}](\mathbf{6})}$}

\section{Data collection}

The data collection was carried out using Mo K $\alpha$ radiation (graphite monochromator) with a frame time of 1 second and a detector distance of $4.00 \mathrm{~cm}$. A collection strategy was calculated and complete data to a resolution of $0.70 \AA$ with a redundancy of 11.38 were collected. Five major sections of frames were collected with $0.50^{\circ} \omega$ and $\varphi$ scans. A total of 1487 frames were collected. The total exposure time was 0.41 hours. The frames were integrated with the Bruker SAINT software package ${ }^{14}$ using a narrow-frame algorithm. The integration of the data using a monoclinic unit cell yielded a total of 101893 reflections to a maximum $\theta$ angle of $28.31^{\circ}$ ( $0.75 \AA$ resolution), of which 8781 were independent (average redundancy 11.604 , completeness $=99.9 \%, \mathrm{R}_{\text {int }}=9.78 \%$, $\left.R_{\text {sig }}=4.50 \%\right)$ and $6582(74.96 \%)$ were greater than $2 \sigma\left(\mathrm{F}^{2}\right)$. The final cell constants of $\mathrm{a}=$ 13.0323(4) $\AA, \mathrm{b}=15.4135(5) \AA, c=18.0620(5) \AA, \beta=103.4350(10)^{\circ}$, volume $=3528.89(19) \AA^{3}$, are based upon the refinement of the XYZ-centroids of 9919 reflections above $20 \sigma(\mathrm{I})$ with $5.114^{\circ}$ $<2 \theta<54.73^{\circ}$. Data were corrected for absorption effects using the Multi-Scan method (SADABS). ${ }^{15}$ The ratio of minimum to maximum apparent transmission was 0.947 . The calculated minimum and maximum transmission coefficients (based on crystal size) are 0.8940 and 0.9680 .

\section{Structure solution and refinement}

The space group $\mathrm{P} 2{ }_{1} / \mathrm{c}$ was determined based on intensity statistics and systematic absences. The structure was solved and refined using the SHELX suite of programs. ${ }^{16-17}$ An intrinsicmethods solution was calculated, which provided most non-hydrogen atoms from the E-map. Fullmatrix least squares / difference Fourier cycles were performed, which located the remaining nonhydrogen atoms. All non-hydrogen atoms were refined with anisotropic displacement parameters. The hydrogen atoms were placed in ideal positions and refined as riding atoms with relative isotropic displacement parameters, with exception of H5n which was refined for all parameters. The final anisotropic full-matrix least-squares refinement on $\mathrm{F}^{2}$ with 420 variables converged at $\mathrm{R} 1=3.91 \%$, for the observed data and $\mathrm{wR} 2=8.70 \%$ for all data. The goodness-of-fit was 1.028 . The largest peak in the final difference electron density synthesis was $0.371 \mathrm{e}^{-} / \AA^{3}$ and the largest hole was $-0.332 \mathrm{e}^{-} / \AA^{3}$ with an RMS deviation of $0.057 \mathrm{e}^{-} / \AA^{3}$. On the basis of the final model, the calculated density was $1.211 \mathrm{~g} / \mathrm{cm}^{3}$ and $\mathrm{F}(000), 1376 \mathrm{e}^{-}$. 
Table S7. Crystal data and structure refinement for $\left[\mathrm{Ph}{ }_{2} \mathrm{~B}\left({ }^{t} \mathrm{BuIm}\right)_{2} \mathrm{FeNHDipp}\right](6)$.

Empirical formula

Formula weight

Crystal color, shape, size

Temperature

Wavelength

Crystal system, space group

Unit cell dimensions

Volume

Z

Density (calculated)

Absorption coefficient

$\mathrm{F}(000)$

\section{Data collection}

Diffractometer

Theta range for data collection

Index ranges

Reflections collected

Independent reflections

Observed Reflections

Completeness to theta $=25.242^{\circ}$

Solution and Refinement

Absorption correction

Max. and min. transmission

Solution

Refinement method

Weighting scheme

Data / restraints / parameters

Goodness-of-fit on $\mathrm{F}^{2 \square}$

Final $\mathrm{R}$ indices [I $>2 \operatorname{sigma}(\mathrm{I})]$

$\mathrm{R}$ indices (all data)

Largest diff. peak and hole
C38 H50 B Fe N5

643.49

light brown block, $0.248 \times 0.216 \times 0.072 \mathrm{~mm}^{3}$

100(2) K

$0.71073 \AA$

Monoclinic, $\mathrm{P} 2{ }_{1} / \mathrm{c}$

$\mathrm{a}=13.0323(4) \AA$

$\mathrm{b}=15.4135(5) \AA$

$\mathrm{c}=18.0620(5) \AA$

3528.89(19) $\AA^{3}$

4

$1.221 \mathrm{Mg} / \mathrm{m}^{3}$

$0.461 \mathrm{~mm}^{-1}$

1376

Venture D8, Bruker

2.080 to $28.310^{\circ}$.

$-17<=\mathrm{h}<=17,-20<=\mathrm{k}<=20,-22<=\mathrm{l}<=24$

101893

$8781\left[\mathrm{R}_{\text {int }}=0.0978\right]$

6582

$99.9 \%$

Semi-empirical from equivalents

0.7457 and 0.7065

Intrinsic methods

Full-matrix least-squares on $\mathrm{F}^{2}$

$\mathrm{w}=\left[\sigma^{2} \mathrm{Fo}^{2}+\mathrm{AP}^{2}+\mathrm{BP}\right]^{-1}$, with

$\mathrm{P}=\left(\mathrm{Fo}^{2}+2 \mathrm{Fc}^{2}\right) / 3, \mathrm{~A}=0.0277, \mathrm{~B}=2.1111$

$8781 / 0 / 420$

1.028

$\mathrm{R} 1=0.0391, \mathrm{wR} 2=0.0779$

$\mathrm{R} 1=0.0647, \mathrm{wR} 2=0.0807$

0.371 and -0.332 e. $\AA^{-3}$ 


\section{$\left[\underline{\mathrm{Ph}}_{2} \underline{\mathrm{B}\left({ }^{t} \mathrm{BuIm}\right)}\right)_{2} \underline{\mathrm{FeN}\left(\mathrm{CH}_{2}\right.} \underline{\mathrm{Ph}) \mathrm{Dipp}](7)}$}

\section{Data collection}

The data collection was carried out using Mo K $\alpha$ radiation (graphite monochromator) with a frame time of 2 seconds and a detector distance of $4.00 \mathrm{~cm}$. A collection strategy was calculated and complete data to a resolution of $0.77 \AA$ with a redundancy of 16 were collected. Ten major sections of frames were collected with $0.50^{\circ} \omega$ and $\varphi$ scans. A total of 2171 frames were collected. The total exposure time was 1.24 hours. The frames were integrated with the Bruker SAINT software package ${ }^{14}$ using a narrow-frame algorithm. The integration of the data using a monoclinic unit cell yielded a total of 156646 reflections to a maximum $\theta$ angle of $26.44^{\circ}$ ( $0.80 \AA$ resolution), of which 9383 were independent (average redundancy 16.695, completeness $=99.8 \%, \mathrm{R}_{\text {int }}=$ $\left.19.62 \%, R_{\text {sig }}=6.73 \%\right)$ and $6081(64.81 \%)$ were greater than $2 \sigma\left(\mathrm{F}^{2}\right)$. The final cell constants of a $=43.175(3) \AA, b=10.8443(9) \AA, c=20.9042(18) \AA, \beta=111.259(2)^{\circ}$, volume = 9121.4(13) $\AA^{3}$, are based upon the refinement of the XYZ-centroids of 5291 reflections above $20 \sigma(\mathrm{I})$ with $4.648^{\circ}$ $<2 \theta<41.63^{\circ}$. Data were corrected for absorption effects using the Multi-Scan method (SADABS). ${ }^{15}$ The ratio of minimum to maximum apparent transmission was 0.827 . The calculated minimum and maximum transmission coefficients (based on crystal size) are 0.9570 and 0.9740 .

\section{Structure solution and refinement}

The space group C2/c was determined based on intensity statistics and systematic absences. The structure was solved and refined using the SHELX suite of programs. ${ }^{16-17}$ An intrinsicmethods solution was calculated, which provided most non-hydrogen atoms from the E-map. Fullmatrix least squares / difference Fourier cycles were performed, which located the remaining nonhydrogen atoms. All non-hydrogen atoms were refined with anisotropic displacement parameters. The hydrogen atoms were placed in ideal positions and refined as riding atoms with relative isotropic displacement parameters. The solvent ether is disordered in a channel (along the crystallographic $c$ axis) with two half ether molecules disordered over special positions and refined with restraints and constraints. The final anisotropic full-matrix least-squares refinement on $\mathrm{F}^{2}$ with 561 variables converged at $\mathrm{R} 1=6.56 \%$, for the observed data and $\mathrm{wR} 2=20.07 \%$ for all data. The goodness-of-fit was 1.037. The largest peak in the final difference electron density synthesis was $0.651 \mathrm{e}^{-} / \AA^{3}$ and the largest hole was $-0.833 \mathrm{e}^{-} / \AA^{3}$ with an RMS deviation of $0.090 \mathrm{e}^{-} / \AA^{3}$. On the basis of the final model, the calculated density was $1.176 \mathrm{~g} / \mathrm{cm}^{3}$ and $F(000), 3472 \mathrm{e}^{-}$. 
Table S8. Crystal data and structure refinement for $\left[\mathrm{Ph}{ }_{2} \mathrm{~B}\left({ }^{t} \mathrm{BuIm}\right)_{2} \mathrm{FeN}\left(\mathrm{CH}_{2} \mathrm{Ph}\right) \mathrm{Dipp}\right](7)$.

Empirical formula

Formula weight

Crystal color, shape, size

Temperature

Wavelength

Crystal system, space group

Unit cell dimensions

Volume

Z

Density (calculated)

Absorption coefficient

$\mathrm{F}(000)$

\section{Data collection}

Diffractometer

Theta range for data collection

Index ranges

Reflections collected

Independent reflections

Observed Reflections

Completeness to theta $=25.242^{\circ}$

Solution and Refinement

Absorption correction

Max. and min. transmission

Solution

Refinement method

Weighting scheme

Data / restraints / parameters

Goodness-of-fit on $\mathrm{F}^{2}$

Final R indices [I $>2 \operatorname{sigma}(\mathrm{I})]$

$\mathrm{R}$ indices (all data)

Largest diff. peak and hole
C49 H66 B Fe N5 O

807.72

light brown block, $0.12 \times 0.09 \times 0.07 \mathrm{~mm}^{3}$

100(2) K

$0.71073 \AA$

Monoclinic, C2/c

$\mathrm{a}=43.175(3) \AA$

$\alpha=90^{\circ}$.

$\mathrm{b}=10.8443(9) \AA$

$\mathrm{c}=20.9042(18) \AA$

9121.3(13) $\AA^{3}$

$\beta=111.259(2)^{\circ}$.

8

$1.176 \mathrm{Mg} / \mathrm{m}^{3}$

$0.371 \mathrm{~mm}^{-1}$

3472

Venture D8, Bruker

1.945 to $26.437^{\circ}$.

$-53<=\mathrm{h}<=53,-13<=\mathrm{k}<=13,-26<=\mathrm{l}<=26$

156646

$9383\left[\mathrm{R}_{\text {int }}=0.1962\right]$

6081

$100.0 \%$

Semi-empirical from equivalents

0.7454 and 0.6162

Intrinsic methods

Full-matrix least-squares on $\mathrm{F}^{2}$

$\mathrm{w}=\left[\sigma^{2} \mathrm{Fo}^{2}+\mathrm{AP}^{2}+\mathrm{BP}\right]^{-1}$, with

$\mathrm{P}=\left(\mathrm{Fo}^{2}+2 \mathrm{Fc}^{2}\right) / 3, \mathrm{~A}=0.0988, \mathrm{~B}=22.1439$

$9383 / 52$ / 561

1.037

$\mathrm{R} 1=0.0656, \mathrm{wR} 2=0.1556$

$\mathrm{R} 1=0.1130, \mathrm{wR} 2=0.2007$

0.651 and -0.833 e. $\AA^{-3}$ 


\section{References}

1. Schwindt, M. A.; Lejon, T.; Hegedus, L. S. Improved Synthesis of (Aminocarbene)chromium(0) Complexes with Use of $\mathrm{C}_{8} \mathrm{~K}-$ Generated $\mathrm{Cr}(\mathrm{CO})_{5}{ }^{2-}$. Multivariant Optimization of an Organometallic Reaction. Organometallics 1990, 9, 2814-2819.

2. Gao, Y.; Carta, V.; Pink, M.; Smith, J. M. Catalytic Carbodiimide Guanylation by a Nucleophilic, High Spin Iron(II) Imido Complex. J. Am. Chem. Soc. 2021, 143, 5324-5329.

3. DiFranco, S. A.; Maciulis, N. A.; Staples, R. J.; Batrice, R. J.; Odom, A. L. Evaluation of Donor and Steric Properties of Anionic Ligands on High Valent Transition Metals. Inorg. Chem. 2012, 51, 1187-1200.

4. Cowley, R. E.; Eckert, N. A.; Vaddadi, S.; Figg, T. M.; Cundari, T. R.; Holland, P. L. Selectivity and Mechanism of Hydrogen Atom Transfer by an Isolable Imidoiron(III) Complex. J. Am. Chem. Soc. 2011, 133, 9796-9811

5. Evans, D. F. The Determination of the Paramagnetic Susceptibility of Substances in Solution by Nuclear Magnetic Resonance. J. Chem. Soc. 1959, 2003-2005.

6. Ion Prisecaru. WMOSS4 Mössbauer Spectral Analysis Software, www.wmoss.org, 20092016.

7. Zhizhko, P. A.; Zhizhin, A. A.; Belyakova, O. A.; Zubavichus, Y. V.; Kolyagin, Y. G.; Zarubin, D. N.; Ustynyuk, N. A. Oxo/Imido Heterometathesis Reactions Catalyzed by a SilicaSupported Tantalum Imido Complex. Organometallics 2013, 32, 3611-3617.

8. Hickey, A. K.; Lee, W. T.; Chen, C. H.; Pink, M.; Smith, J. M. A Bidentate Carbene Ligand Stabilizes a Low-Coordinate Iron(0) Carbonyl Complex. Organometallics 2016, 35, 30693073.

9. Neese, F. Software Update: the ORCA Program System, Version 4.0. WIREs Comput. Mol. Sci. 2018, 8, e1327.

10. Weigend, F.; Ahlrichs, R. Balanced basis sets of split valence, triple zeta valence and quadruple zeta valence quality for $\mathrm{H}$ to Rn: Design and assessment of accuracy. Phys. Chem. Chem. Phys. 2005, 7, 3297-305.

11. Pantazis, D. A.; Chen, X. Y; Landis, C. R.; Neese, F. All-Electron Scalar Relativistic Basis Sets for Third-Row Transition Metal Atoms. J. Chem. Theory Comput. 2008, 4, 908-919.

12. Grimme, S.; Ehrlich, S.; Goerigk, L. Effect of the Damping Function in Dispersion Corrected Density Functional Theory. J. Comput. Chem. 2011, 32, 1456-1465. 
13. Grimme, S.; Antony, J.; Ehrlich, S.; Krieg, H. A Consistent and Accurate $a b$ initio Parametrization of Density Functional Dispersion Correction (DFT-D) for the 94 Elements H-Pu. J. Chem. Phys. 2010, 132, 154104(1-19).

14. SAINT v. 2018.1, Bruker AXS, Madison, WI, 2018.

15. SADABS v. 2018.1, Bruker AXS, Madison, WI, 2018.

16. Sheldrick, G. M. SHELXT-Integrated Space-Group and Crystal-Structure Determination. Acta Cryst. 2015, $A 71,3-8$.

17. Sheldrick, G. M. Crystal Structure Refinement with SHELXL. Acta Cryst. 2015, C71, 3-8. 